\title{
Footnotes : anatomical and functional studies on the sole of the foot
}

Citation for published version (APA):

Weijers, R. E. (2005). Footnotes : anatomical and functional studies on the sole of the foot. [Doctoral Thesis, Maastricht University]. Datawyse / Universitaire Pers Maastricht.

https://doi.org/10.26481/dis.20051028rw

Document status and date:

Published: 01/01/2005

DOI:

10.26481/dis.20051028rw

Document Version:

Publisher's PDF, also known as Version of record

\section{Please check the document version of this publication:}

- A submitted manuscript is the version of the article upon submission and before peer-review. There can be important differences between the submitted version and the official published version of record.

People interested in the research are advised to contact the author for the final version of the publication, or visit the DOI to the publisher's website.

- The final author version and the galley proof are versions of the publication after peer review.

- The final published version features the final layout of the paper including the volume, issue and page numbers.

Link to publication

\footnotetext{
General rights rights.

- You may freely distribute the URL identifying the publication in the public portal. please follow below link for the End User Agreement:

www.umlib.nl/taverne-license

Take down policy

If you believe that this document breaches copyright please contact us at:

repository@maastrichtuniversity.nl

providing details and we will investigate your claim.
}

Copyright and moral rights for the publications made accessible in the public portal are retained by the authors and/or other copyright owners and it is a condition of accessing publications that users recognise and abide by the legal requirements associated with these

- Users may download and print one copy of any publication from the public portal for the purpose of private study or research.

- You may not further distribute the material or use it for any profit-making activity or commercial gain

If the publication is distributed under the terms of Article $25 \mathrm{fa}$ of the Dutch Copyright Act, indicated by the "Taverne" license above, 
FOOTNOTES 
ISBN 9052784876

Copyright(c) R.E. Weijers, Maastricht 2005. All rights reserved.

The copyright of articles that have been published or accepted for publication has been transferred to the respective journals.

Lay-out: I. Kengen

Printed by: Datawyse / Universitaire Pers Maastricht 


\title{
FOOTNOTES \\ anatomical and functional studies on the sole of the foot
}

\author{
Proefschrift
}

ter verkrijging van de graad van doctor aan de Universiteit Maastricht, op gezag van de Rector Magnificus,

Prof. mr. G.P.M.F. Mols,

volgens het besluit van het College van Decanen,

in het openbaar te verdedigen

op vrijdag 28 oktober 2005 om 16.00 uur

door

René Erik Weijers

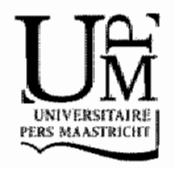




\section{Promotores}

Prof. dr. J.M.A. van Engelshoven

Prof. dr. H. van Mameren

\section{Copromotor}

Dr. G.J. Kemerink

\section{Beoordelingscommissie}

Prof. dr. R.G.T. Geesink (voorzitter)

Prof. dr. D.M.F.M. van der Heijden

Prof. dr. H.W.J. Huijskens, Technische Universiteit Eindhoven

Dr. M. Maas, Universiteit van Amsterdam

Dr. N.C. Schaper 
Voor mijn dierbaarsten 



\section{Contents}

Chapter 1 Introduction and aim of the thesis 9

Chapter 2 Functional anatomy of the sole of the forefoot: MRI compared 13

with current anatomical descriptions

Submitted

Chapter 3 Changes of the soft tissue of the forefoot during loading: a volumetric study

The Foot 2003; 13: 70-75.

Chapter 4 The relationship of the position of the metatarsal heads and peak plantar pressure

Foot and Ankle International 2003; 24: 349-353

Chapter 5 Plantar pressure and sole thickness of the forefoot

Foot and Ankle International, accepted for publication

as a source of error

Foot and Ankle International 2005; 26: 287-393

Chapter 7 The effect of the tube angulation on the measurement of intermetatarsal angles

Joumal of the American Podiatric Medical Association 2005: 95: $370-375$

Chapter 8 The effects of repeated low velocity impacts on the mechanical properties of the heel pad

Submitted

Chapter 9 The damping properties of the venous plexus of the heel region of the foot during simulated heelstrike Journal of Biomechanics, in press

Chapter 10 Pendulum impact testing on the forefoot: a comparison with the heel

Submitted

Chapter 11 General discussion and summary

Algemene beschouwing en samenvatting

Curriculum vitae

Dankwoord 


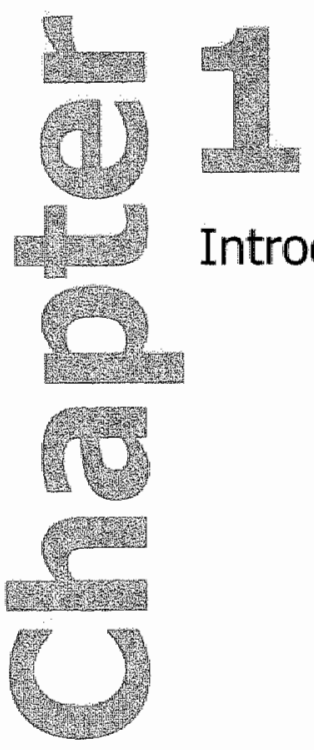

Introduction and aim of this thesis 
12. Wernett PJ and Duplock LR: Pressure distribution beneath the human foot. I Am Podiatr Med Assoc 1993; 83: 674-678.

13. Cavariagh PR, Rodgers MM and liboshi A: Pressure distribution under symptom-free feet during barefoot standing. Foot Ankle 1987; $7: 262-276$.

14. Luger $\mathrm{EJ}_{i}$ Nissan $M$, Karpf $\mathrm{A}_{x}$ et al. : Pattenis of weight distribution under the metatersal heads $\mathrm{J}$ Bone Joint Surg Br $1999 ; 81: 199-202$.

15. Toung CR: The F-SCAN system of foot pressure analysis. Clin Podiatr Med Surg 1993; 10:455-461.

16. Fuller $E$ and Hogge JD. Measurement of the expansion of the calcaneal fat pad upon weightbearing. I Am Podiatr Med Assoc 19g8; 88: 12-16;

17. Pridhasuk S; The heel pad in phantar heel pain. J Bone Joint Surg Br 1994; 76: 140-142.

18. De clerca $D$, Aerts $P$ and kunnen fin. The medhanical charactieristics of the human heel pad during foot strike in nuning: an in wivo oneradiographic study. J Elomeon 1994; $27: 1213-1222$.

19. Prichasuk $S$, Mulpruek $P$ and Sirwongpairat $P$ : The hed-pad compressibility. Clin Othop 1994; 197-200.

20. Kanatl $U$, Yetkin $H$, Simsek $A_{n}$ et al: The relationiship of the heel pad compressibility and plantar pressure distribution, Foot Arikle Int 2001; 22; 662-665.

21. Morag E and Cawaragh PR: Structural and finctional predictors of regional peak pressures under the foot during walking. J Bomech $1999 ; 32: 359-370$.

22. Rome K, Campbenl $R$, Fint $A$, et al: Reliabilty of weight-bearing heel pad thickness measumements by ultrasound. Cin Biomech (Bristol, Avon) 1998; 13: 3744375 .

23. Rome K, Campbell PS, Flint AA, et al.: Ultrasonic heel pad thickness measurements: a preliminary study. Br ] Radiol 1.998; 71:1149-1152.

24. Cawanagh PR: Plantar soft tssue thickness during ground contart in walking. I Biomech 1999; 32: 623-628.

25. Hermann B: Form and structure of the metatarsall head arch in adults. Ultrasonographic and podometic studies. 2 Otthop Ihre Grenzgeb 1995; 133: 335-34t0.

26. Hsu $T C$, Wang $C_{\text {, }}$ Tsai $W_{C}$, et al.: Comparison of the mechanical properties of the heel pad between young and elderty adults. Arch Phys Med Rehabil 1998; 79; 1101-1104.

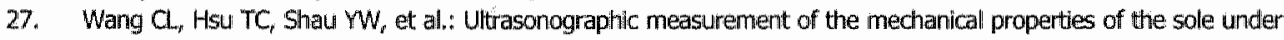
the metatarsal heads. J Orthop Res $1999 ; 174709-713$.

28. Jongensen $U$ and Bojsen-Moller $F$ : Shock absorbency of factors in the shoe/heel interaction with speciall focus on role of the heel pard. Foot Ankle 1989; 9: 294-299.

29. Aests P and De Clerca D: Deformation characteristics of the heel region of the shod foot during a simulated heel strike: the effect of varying midsole hardness. I Sports Sai 1993; 11: 449-461.

30. Aerts P, Ker RF, De Clencq $\mathbb{D}_{\text {, et }}$ al.: The mechanical properties of the human heel pad: a paradox resolved. 1 Biomech 1995; 28: 1299-1308.

31. Kinoshita $H_{1}$ Francis PR, Murase $T$, et al.: The mechanical properties of the heel pad in elderly adults. Eur J Appl Physiol Occup Physiol 1996; 73: 404-409.

32. Ker RF: The time-dependent mechanical properties of the human heel pad in the context of loconwotion. J Exp Biol 1996; 199 (PA 7): 1501-1508.

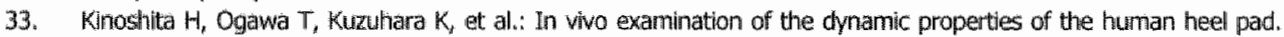
int J Sprorts Med 1993; 14:312-319.

34. Bennett ME and Ker RF: The medhanical properties of the humali subcalcaneal fat pad in compression, J Anat $1.990 * 171: 131-138$.

35. Nigg BM and Lu W: The effect of musde stiffness and damping on simulated impact force peaks during running. I Blomech 1999; 32: $849-856$.

36. Pain MT and Challis JH: The mole of the heel pad and shank soft tissue during impacts: a further resolution of a paradox. I Bomech 2001; 34: 327-333.

37. Valiant GA: Transmission and attenuation of heelstrike acoelarations. In Biomechanics of distance nunning, edited by Cavanagh PR. Champaign, Ilinois; Human Kinetics. Publishers, Inc. 1990. 225-247.

38. Miller-Young JE, Duncan NA and Baroud G: Material properties of the human calcaneal fat pad in compression: expertiment and theory. J Biomech 2002; 35: 1523-1531.

39. Wakeling JM, Von Tschamer $V$, Nigg BM, et al.. Musde adtivity in the leg is tuned in response to ground reaction forces. J Appl Pthysict 2001; 91 : $1307-1317$.

40. Wakeling JM and Nigg BM: Modification of soft tissue vibrations in the leg by muscular activity. J Appl Physiol 2001; 90: $4: 12-420$.

41. Boyer KA and Migg BM: Musde activity in the leg is tuned in hesponse to impact force characteristips. I Biomech 2004: $37: 1583-1588$.

42. Nigg BM and Wakeling JM: Impact forces and imuscle tuning; a new paradigm. Exerc Sport So Rew 2001; 29: $37-41$. 

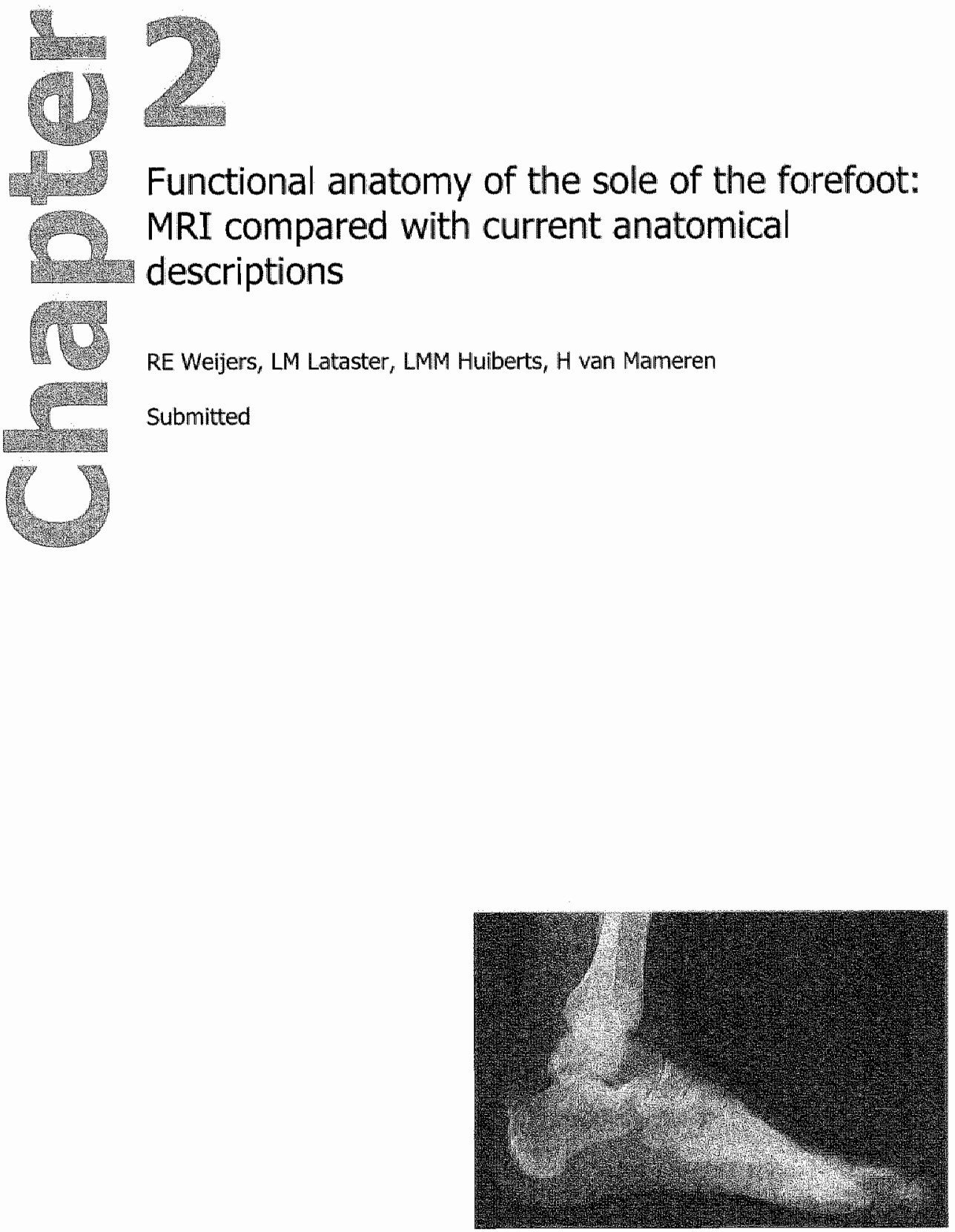


\section{Abstract}

The current concepts on the (functional) anatomy of the sole of the forefoot largely originate from dissection and histology. However, dissection is such a difficult process, that the presentations of the anatomy differ considerably among publications Aternatively, the internal structure of the sole can be visualized with Magnetic Resonance Iinaging (MRI), In this MRI-study, we compare the current anatomical descriptions with the anatomy as depicted on MRI. Moreover, we try to visualize the effect of shear and torque on the sole of the forefoot with MRI of six cadaver feet, high-resolution MR images were obtained for descriptive purposes. For functional anatomy, three healthy volunteers were scanned while stressing the sole. The MRI and current anatomical descriptions are largely in accord, The most important differences seem to be (1) the extension of the Subcutaneous Fibrous Layer beyond the heel region and its connections with the superficial Stratum of the Plantar Aponeurosis in the forefoot and (i) the configuration of the Plantar Interdigital Ligament with ils connection at the lateral foot border. As MRI depicted, the Iongitudinal shear force changes (i) the orientation of the Plantar Interdigital Ligament, (ii) the position of the mass of the sole of the foot in relation to the metatarsal heads, and (1i) the appearance of the Plantar Aponeurosis. We were unable to detect consistent changes in the sole on torque. The results add to a sounder description and a better understanding of the functional anatomy of the sole of the forefoot. 


\section{Introduction}

During locomotion, the sole of the foot is not only expased to vertical forces but also to shear and torque. These forces tend to slide the skin relative to the deeper structures of the foot with the risk of damaging vulnerable structures such as the nerves and arteries. Therefore, the subcutaneous tissue in the sole of the foot should anchor the skin and protect the vulnerable structures. With this in mind, several investigators described the internal architecture of the sole of the foot and its relation to the deeper structures of the foot such as the Plantar Aponeurosis ${ }^{1-9}$. These descriptions were mainly based on dissectional work and histology. However, dissection is a difficult process, in which the anatomist has to decide whether or not to dissect a structure to reach a deeper layer of the specimen. Therefore, the result will partly depend on his expectations. This especially holds for the reticular pattern of the internal fibrous structure of the sole of the forefoot. The varying presentation of the anatomy of the sole in rellation to the plantar aponeurosis in different atlases, textbooks, and illustrations in scientific papers bears witness to this. Histology provides detalled anatomical information focally, but is less useful in obtaining a global description of the anatomy.

Magnetic Resonance Imaging (MRI) can visualize the internal structure of the sole of the foot without affecting its integrity. Although MRI avoids the intrinsic problems of dissectional anatomy and histology, the spatial resolution and typification of tissue are worse. In this report, we will study the anatomy of the sole of the forefoot by means of MRI with the purpose to correlate the current anatomical descriptions with the anatomy as depicted on MRI. Moreover, we will investigate whether MRI can visualize the effect of shear and torque on the sole of the forefoot. The results are expected to add to a sounder description and a better understanding of the functional anatomy of the sole of the forefoot.

\section{Current description of anatomy}

In this paragraph we describe the current opinion on the anatomy of the sole of the forefoot, based on several sources ${ }^{2,8}, 9$. In this section we want to set the scene for our findings and define the nomenclature we are about to use. To avoid confusion, we will use the following terms: proximal: the heel side; distal: the toe side; plantar: the side of the sole; dorsal: the side of the dorsum of the foot; transverse: the plane perpendicular to the second metatarsal shaft; axial: plane parallel to the sole of the foot. The illustrations are all T1-weighted MRI irmages. In the accompanying drawings, 'white" refers to structures of current anatomy, 'black' refers to the differences with MRI as mentioned in Results, 'light grey' to bones and "dark grey" to tendons.

We define the sole of the foot as all the tissue between and including the Plantar Aponeurosis and the plantar skin. Basically, the sole of the foot consists of adipose tissue, which is pervaded by fine but collectively strong connective tissule. This connective tissue tethers the skin and limits the displacement of fat. In regions of high stress, it is organized into strands. The direction of these strands is adapted to the prevailing stress in that region. This principle was demonstrated in the sub-metatarsal region ${ }^{\text {? }}$

In the heel region, the vertical component of impact force dominates the loading. The damping properties of the heel pad are said to originate from the arrangement of fibrous septae separating the subcutaneous fat into compartments ${ }^{6}$. A fibrous layer or "internal fibrous cup" (which we will call the Subcutaneous Fibrous Layer) divides the adipose tissue of the heel pad into a superficial part with micro-chambers and a deep part with macrochambers (Figs. 1 and 2A). The fibrous septae of the superficial, outer part are rich in elastic fibres and they extend from the reticular dermis to the internal fibrous cup. The fibrous septae of the deep, inner, part are rich in collagen ${ }^{10}$. They spirally connect the fibrous internal cup to the periosteum of the calcaneus. The twisted macro-chambers are subdivided into smaller compartments by delicate septae rich in elastic fibres. The Plantar Aponeurosis is 


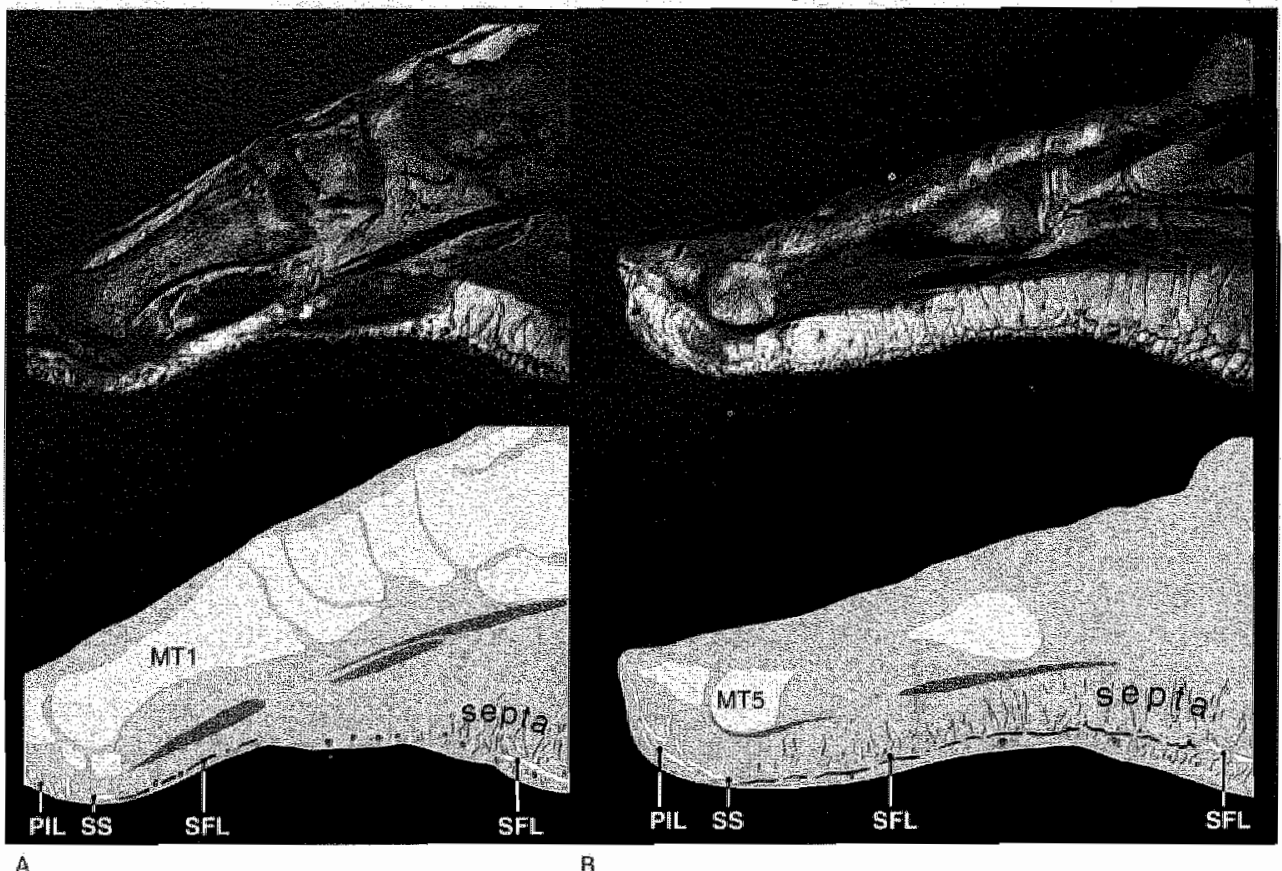

Fig. 1: sagital reconstructions of the medial (Fig. 1A) and lateral (Fig. 1B) foot border through the first and fifth metakasal bone (MT 1 and 5 ). The Subcutaneous Fibrous Layer (SFL) is a reticulated structure that divides the basic pattern of vertical connective tissue septae throughout the subcutis of the sole, mo deep matro and superflcial micro-thamber's. The Subcutaneous Fibrous Layer extends fron the theel to the metatarsal heads where it merges with the Stperficial Stratum (SS) of the Plantar Aponeurosis (PA). The superficial layer of microm chambers gradually thins until, at this level of the metatarsal heads, only little fat is present between the Subcutanegus Fibrous Layer and the skin. At the heel pad and Iateral foot border, the Subcutaneous Fibrous Layer was most consistently present. At the medial arch of the foot, it was thinmer and at smaller areas even absent. Distal in the forefoot, the Plantar Interdigital Ligament (PIL) merges with the Superficial Stratum. A mooring ligament was mot discernable.

a deep border of the solle. It is divided in a medial, central and lateral part. Here, we will only discuss the central part that is the strongest and thickest part (Fig. 2). The Plantar Aponeurosis diverges from the medial process of the calcaneal tuberosity toward the toes. Proximal to the metatarsal heads, the central part divides into five bands. Only proximally in this area, transverse fibres unite the five diverging bands. Distally, the five bands are said to split up into five superficial and ten deep tracts, making up a Superficial and a Deep Stratum (Fig. 2B and 3).

In the Superficial Stratum of the Plantar Aponeurosis, the superficial tract of each band connects with the dermis: the skin ligaments. At the level of the metatarsal heads the separation with the rest of the Plantar Aponeurosis is completed. Distal to the metatarsal heads, they reach the skin of the sole of the forefoot. Two of these tracts course to the sides of the foot, two others to the interstice between the toes 1-2 and 4-5 and one to the base of the third toe. No fibres reach the skin distal to the skin crease between the base of the toe and the sole.

In the Deep Stratum of the Plantar Aponeurosis, two deep tracts originate from each digital band of the aponeurosis. Each pair of tracts takes an oblique dorso-distal course and passes allong the sides of the flexor tendons. Deeply, the tracts connect the aponeurosis with the fascia of the transverse head of the adductor hallucis, the interosseous fascia, the periosteum, the deep transverse metatarsal ligaments, the plantar ligament of the 
metatarsophalangeal joints, and fibrous flexor sheaths at the base of each proximal phalanx. These tracts separate the flexor tendons from the lumbrical muscles, digital neves and vessels and they fix them to the metatarsal heads.

In the four spaces between adjacent metatarsal heads and the bases of the proximal phalanges, adipose tissue cushions the digital nerves and vessels from plantar pressure: the fat bodies (Fig. 3C). Vertical strands of collagen fibres stabilize the bodies sideways. These strands extend from the side of the fibrous sheath of the flexor tendons to the skin through the Superficial Stratum of the Plantar Aponeurosis. Some of the fibres arch below the flexor sheet enclosing fat plantar to the tendons. Thus, a cushion for the tendons is formed.

In the area in which the Plantar Aponeurosis gives off the Deep Stratum, a series of Subcutaneous Transverse Bands are mentioned to be formed. The Subcutaneous Transverse Bands should contain fibres that run an oblique course anteriorly and deeply from the dermis, penetrate the Plantar Aponeurosis, and continue via the sagittal septae and the deep fasciae to the bases of the proximai phalanges.

Just distal to the metatarsal heads, six to eight transverse lamellae separated by fat extend across the sole of the forefoot: the Plantar Interdigital Ligament (superficial transverse metatarsal ligament or natatory ligament) (Fig. 4). At the plantar side, this ligament merges with the deep aspect of the Superficial Stratum of the Plantar Aponeurosis (Fig. 1). At the dorsal side, it merges with the fibrous flexor sheaths and with the mooring ligament. The mooring ligament is said to run deep to the flexor tendon sheaths, connecting the adjacent phalanges.

Thus, the central part of the Plantar Aponeurosis provides an intermediary between the skin and the skeleton of the foot, through numerous cutaneous retinacula and deep septae extending anteriorly to the metatarsals and phalanges.

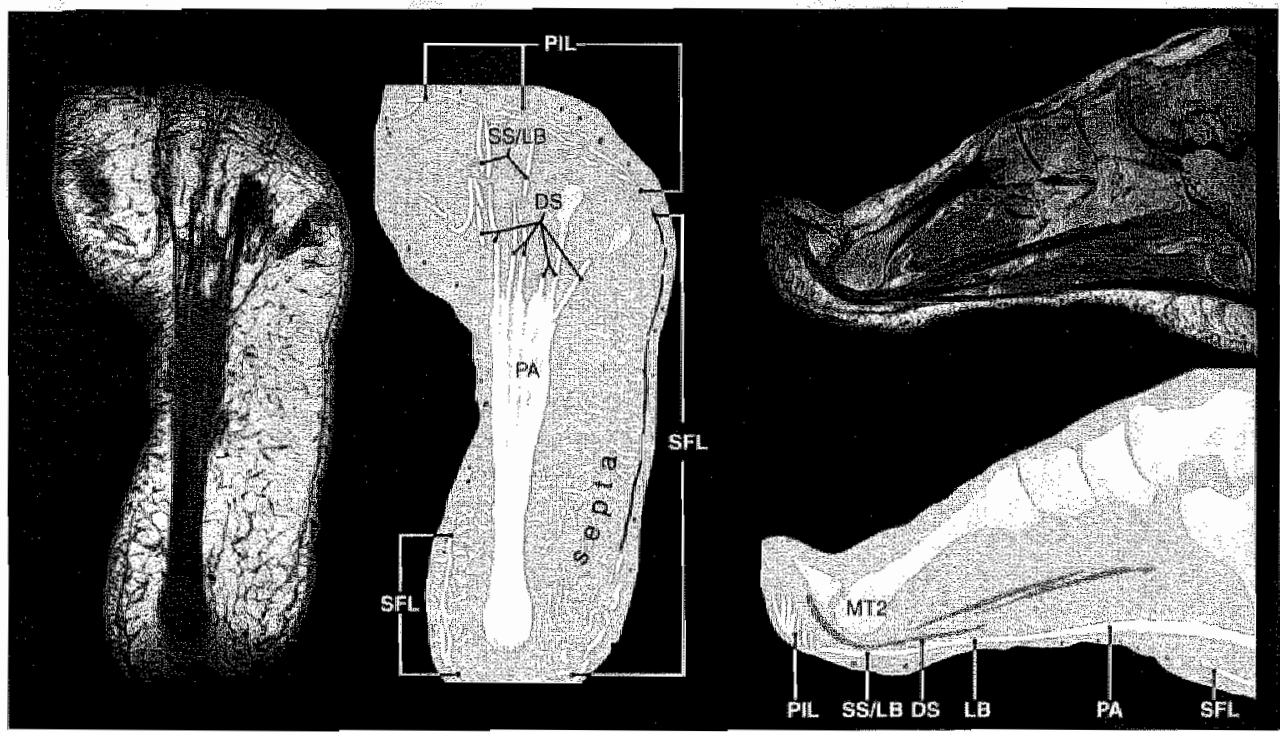

A

B.

Fig. 2: Cuned reconstructions of the central part of the Plantar Aponeurosis (PA) in the axial plane (Fig. $2 A$ ) and the sagittat plane through the second ray (MT 2) (Fig. 2B). The longitudinal fibres of the central aponeurotic part condense into five Longitudinal Bands (LB) that are not separately discemable proximaliy, because of the transverse fibres that unite the bands (Fig. 2A), see also Figure 6. Distally, current anatomy describes the Longitudinal Band to split up into the Deep Stratum (DS) and the Superficial Stratum (5S). MRT depicts the Longitudinal Bands as continuous from their otigin to the ball of the foot, giving off the septate of the Deep and the Superficial Stratum (Fig. 2B). Asso note the Subcutaneous Fibrous Layer (SFL), and its connections with the Plantar Interdigital Lgament (PIL) at the fifth metatarsal head (Fig. $2 \mathrm{~A}$ ). The Subcutareous Transyerse Bands are not discernable on the MRI-images. 


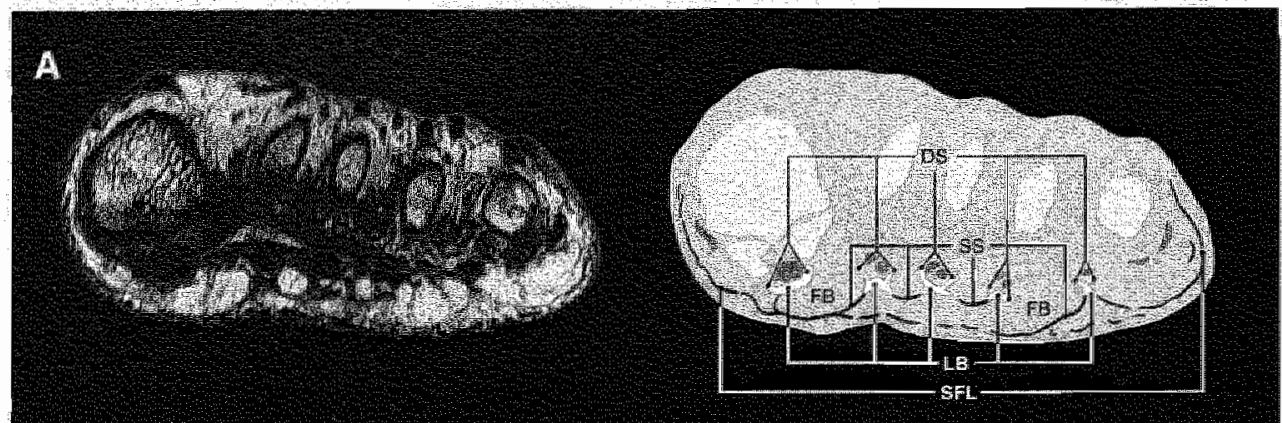

B
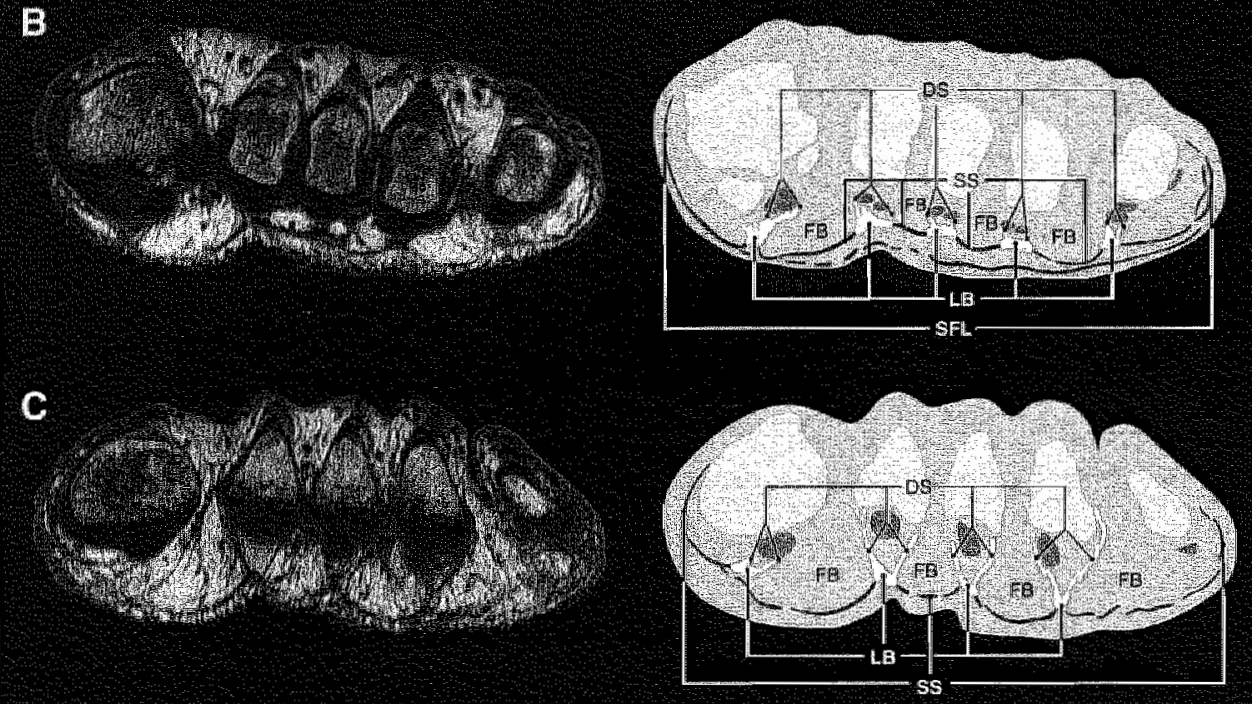

Hig. 3: Reconstructed transwerse images proximal (Flg. 3A. at (Fig. 3B), and distal (Fig. 3C) to metatarsophanged joints. To adjut for the diffarent length of the metatarsals the reconstructions are curved through the metatarsophatangeal joints. The Longitudinal Band (LB) is gradually positioned more superficially as

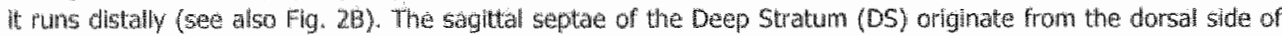
the band and endose the flexor tevidons on crie side and the fat boties (fB) at the other. Eecause the strands of Collagen fores that originate from the fibrous sheath (Fig. 38 and $3 \mathrm{C}$ ) of the flexor tendon camot be diferentiated from the sepha of the Deep Stratum that originate from the Longtudinal Bands (Fig $3 A$ ). together they are visualized as a continuous tunnel for the ftexor tendons. The Superficial Stratum (SS) is a fanlike structure in which the Longliwdinal Bands stand out as reinforcements. The Superfich stratum tethers the skin of The sole of the forwoot and encloses the fat bodies on the plantar side. The Subcutaneous fibrows Layer (SFL) proximatly lies superhicial to the Superficial stratum and the Longitudinal Bands until it merges distaly.

\section{Materials and Methods}

Two lines of experiments were followed. In the first line, cadaver feet were imaged with a MRI-scanner for anatomical purposes. In the second line, we imaged the foot of subjects while they were stressed for functional purposes. 


\section{Descriptive anatomy}

The right forefoot of six cadavers fixed with formalin was imaged with a 1.5 Tesla MRIscanner (Gyroscan Intera, Philips, Best, The Netherlands). We used T1-welighted 2D-SE sequences in three orthogonal planes (30 to 60 slices; 6 to 10 signal averages; thickness $1.0 \mathrm{~mm}$; field of view $140^{2} \mathrm{~mm}_{s}$ matrix $1024^{2}$ and a scan percentage of $80 \%$; voxel size $0.14 * 0.17 * 1.0 \mathrm{~mm}$ ). Because the contrast between the fat and the connective tissue showed to be superiar on the T1-weighted 2D-SE, we decided not to use 3D sequences. To verify the structures found on MRI, we dissected the sole in two cases. In one case, the foot was sectioned in the sagiltal plane using a band saw. For multi-planar reformats of the sequences, we used EasyScill 4.2.1 software (Philips, Best, The Netherlands).

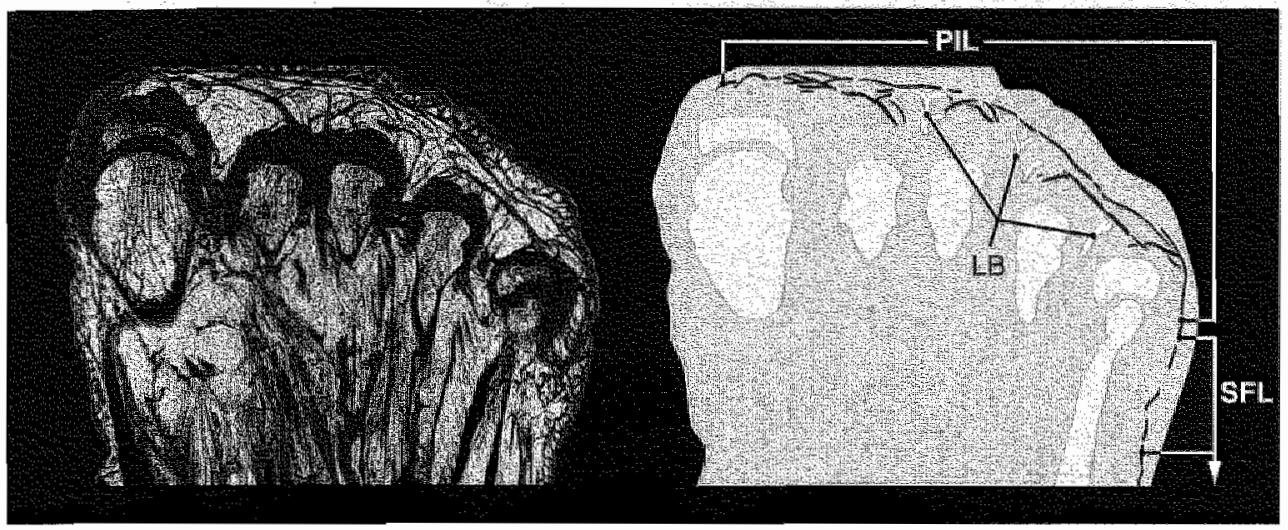

Fig. 4: Axiall image displaying the Plantar" Interdigital Liganemt (PIL). The Plantar Interdigital Ligament consists of a mesh of multiple interconnected short septae that runs across the sole of the forefoot distal to netatarsal heads. At the lateral foot border, the Piantar Interdigital Ligament is conthuous with the Subcutaneous Fibrous Layer (SFL). The fusion of the Longitudinat Bands (LB) with the plantar Interdigital Ligament is visible at the second to the fith ray.

\section{Functional anatomy}

The right forefoot of three healthy subjects was imaged to visualize the effects of shear and torque on the arrangement of the internal connective tissue structures. The imaging was performed in accord with the guidelines of our institutional review board. All subjects gave informed consent. The subjects were supine with the knee flexed. Double-sided adhesive tape fixed the skin of the forefoot to the supporting surface. The mid- and hindfoot were free. Of each subject, four T1 weighted 2D-fast SE scans of the stressed forefoot were made (TR/TE 425/ 8.6 msec; echo train length 7; 12 slices; thickness $1.5 \mathrm{~mm}$; field of view $130^{2} \mathrm{~mm}$; matrix $512^{2}$ and a scan percentage of $80 \%$; voxel size: $0.25 * 0.32 * 1.5 \mathrm{~mm}$ ). Two scans in the sagittal plane through the second metatarsal were made to visualize the effect of shear. Once the subject shifted the foot distally (as in braking during locomotion) and once proximally (as in propulsion), till the moment the adhesive tape tended to give way. In both conditions, the forefoot was imaged and the images were visually compared.

The other two scans were made parallel to the plantar skin to visualize the effect of torque. Once, the subject rotated the forefoot by shifting the heel medially, and once laterally. The difference between the two positions was about 30 degrees. Again, in both conditions, the forefoot was imaged and the images were visually compared. 


\section{Results}

\section{Descriptive anatomy}

The MRI on cadaver feet largely agreed with the current anatomical descriptions. Nevertheless, differences were noted.

\section{The Subcutaneous Fibrous Laver}

At the heel pad only, the Subcutaneous Fibrous Layer was described ${ }^{1}$.11. We identified this layer as reticulated connective tissue that gradually approached the plantar skin from the heel pad to the level of the metatarsal heads, where the layer eventually merged with the Superficial Stratum of the Plantar Aponeurosis (Figs. 1 and $2 A$ ). At the footborders, it loosely merged with the periosteum of the metatarsals 1 and 5 , and the dorsal fascia covering the borders of the foot (Fig. 5). The subcutaneous Fibrous Layer was consistently seen at the tread of the foot. We noted considerable inter-individual differences.

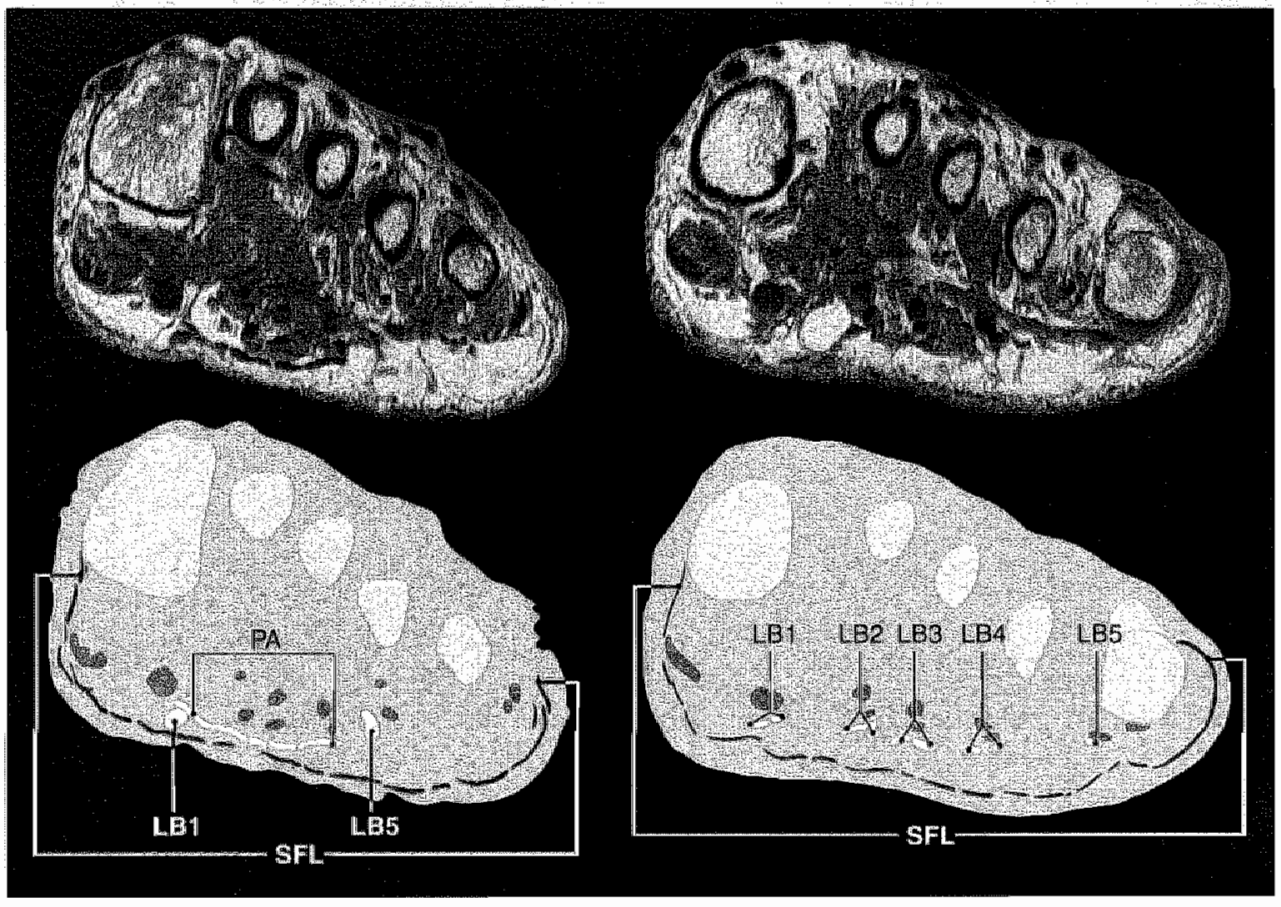

A

B

Fig. 5: Transverse images proximal to (Fig. 5A) and at the level of the fifth metatarsal head (Fig. 5B). At the medal and lateral foot border, the Stbcutaneous fibrous Layer (SFL) connects with the periosteum of the metatarsals and the dorsal fascia of the foot. Proximally (Fig. 5A), the Longitudinal Bands of the first (LB 1 ) and the fith ray (LB 5) atready have separated from the central bands of the Plantar Aponeurosis (PA) that are more or lass still united by the hanswerse fibers. Mone distally (Fig. 58), all angiturinal Bands are separated $(10105)$

\section{Plantar Aponeurosis}

The plate-like central part of the Plantar Aponeurosis condenses into five Longitudinal Bands. In the area where the bands begin to diverge towards the toes, the five bands are united by transverse fibres of the Plantar Aponeurosis (Flgs. 2A and 5). At this level, the Subcutaneous Transverse Bands should be visible. Although MRI displayed subcutaneous septae in this region, no special (transverse) orientation or increased thickness of the septae was visible (Figs. 1, 5A, 
and 6). Only distally to this region, proximal to the metatarsal heads, we could discern thin transverse sheets within the fat bodies, that curved distally as they blended in with the Deep Stratum, thus bounding the metatarsal fat bodies proximally (Fig, 6). These deep transverse sheets matched the current description of the deep part of the Subcutaneous Transverse Band that penetrated the Plantar Aponeurosis ${ }^{2}$.

Currently, the five longitudinal bands are said to split up into five superficial and ten deep tracts 2. The split is not visible with MRI. MRI only displays five Longitudinal Bands that extended distally, until they turn transversely into the Plantar Interdigital Ligament (Fig. 4), Both the Superficial and the Deep Stratum originate from the Longitudinal Bands (Fig. 3).

On the plantar side, different from current anatomy, MRI displayed the Superficial Stratum as continuous fan like structure that spanned the width of the forefoot, in which the Longitudinal Bands (currently called 'tracts of the Superficial Stratum') stood out as reinforcements. Between the reinforcing bands, the thinner part of the fan 'sagged' to the plantar side (Fig. 3). At the level of and distal to the metatarsophalangeal joints, the Superficial Stratum superficially paralleled the skin and merged with the plantar part of the transversely oriented Plantar Interdigital Ligament (Figs, 1 and 2B and 7).

On the dorsal side MRI showed that two sagittal septae of the Deep Stratum originated from each of the five Longitudinal Bands. Unlike the current anatomical description, we could not discern the deep parts of the Plantar Aponeurosis from the sagittally oriented fibres that originated from the tendon sheaths (Fig. 3C). In fact, both structures together formed a continuous sagittal septum that started at the level of the division between the Superficial and Deep Stratum and ended distal to the metatarsophalangeal joints, where it joined the dorsal pait of the Plantar Interdigital Ligament. A pair of septae enfolded the flexor tendons to anchor the tendons to 'the connective tissue box' of the metatarsal heads (Fig. 3). We used the expression 'the connective tissue box' because with MRI the constituting elements (the plantar plate, the metatarsal collateral ligaments, the deep transverse metatarsal ligament, and the connections to the base of the proximal phalanx) were not discemable as separate structures ${ }^{12,13}$.

\section{Plantar Interdigital Ligament}

In three aspects, the current anatomical description and the MRI results of this ligament differed (Fig. 4):

Although the Plantar Interdigital Ligament as a whole did run across the sole of the forefoot, its constituting individual sheets are shorter. Being interconnected, the sheets participate in a transversely orientated mesh that can be observed distally to the metatarsal heads from the medial to the lateral foot border.

The lateral edge of the Plantar Interdigital Ligament does not end freely, but merges with the Subcutaneous Fibrous Layer antero-lateral to the fifth metatarsal head. Thus, the lateral foot boifder directly connects to the sole of the forefoot. On the medial side, the ligament ends freely. Dorsally the sheets reach the base of the toes and the flexor tendons. However, MRI cannot consistently depict the mooring ligament as a separate reinforcing structure.

\section{Fat bodies}

Although the borders of the fat bodies follow from the foregoing, we describe them here to illustrate the internal architecture of the sole of the forefoot from a different perspective. Proximally, the fat bodies reached all the way to the curved deep extensions of the subcutaneous fibrous bands (Fig. 6). Dorsally, the fascia of the deep musculature and the connective tissue boxes of the metatarsals bounded the fat bodies (Fig. 3). The sagittal septae of the Deep Stratum bordered the fat bodies sideways. The Superficial Stratum boxed in the fat bodies on the plantar side. Distally, the most proximal septae of the Plantar Interdigital Ligament closed the compartment (Fig. 4). Of all the central four fat bodies, the one between the first and second ray was the largest. 


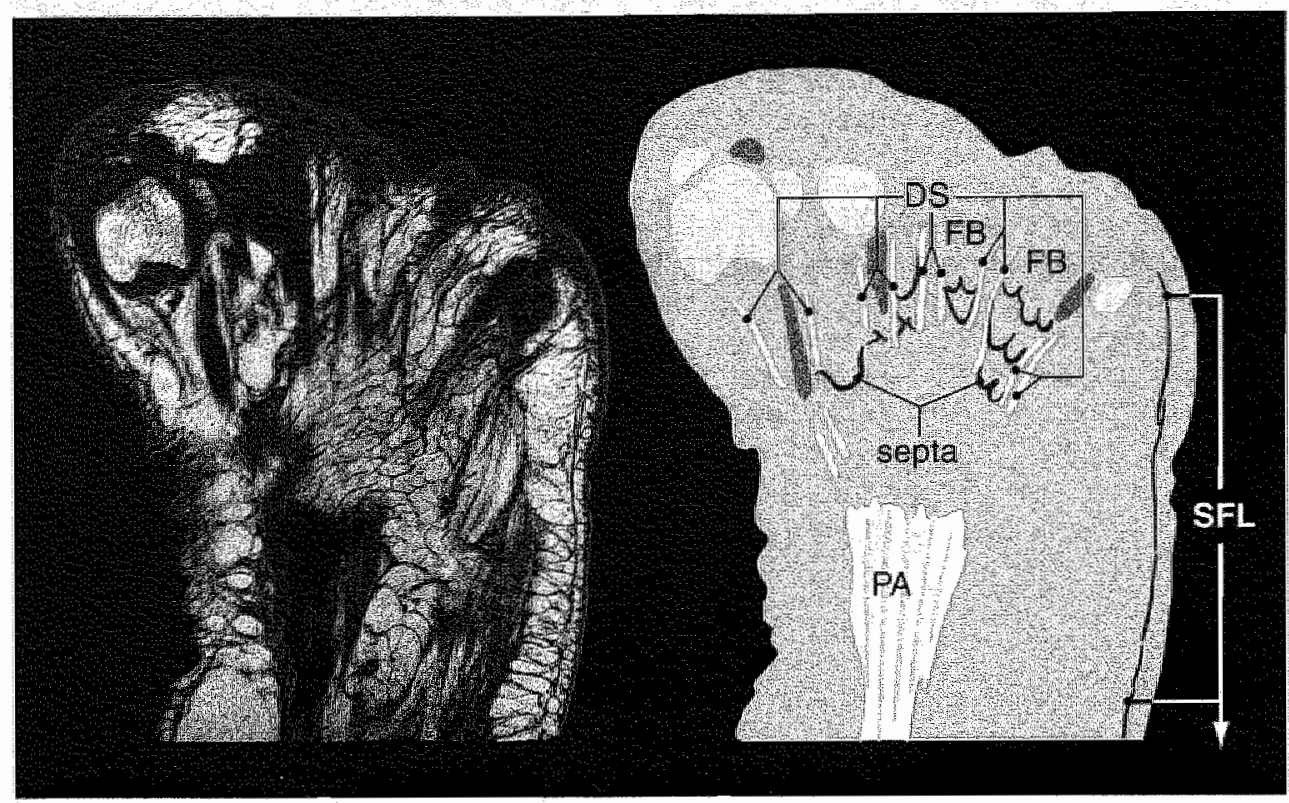

Fig. 6: Axal image displaving the fat bodies (FB). In the lat bodies, thin, curved septae stand out that may represent the deep extensicns of the Supenficial Transverse Bands as can be identified with dissection. The septats also border the fat bodies at the postertor side. Sidewards, the septere mierge with the adjacent Deep Stratum (DS) of the Plantar Aponeurosis (PA). The Subcutaneouls Transverse Bands are not discermable on the MRI-mages. (SFL: Subrutameous Fibrous Layer).

\section{Functional anatomy}

Longitudinal shear on the sole of the forefoot changes (i) the orientation of the Plantar Interdigital Ligament, (ii) the position of the mass of the sole of the foot in relation to the metatarsal heads, and (iii) the appearance of the Plantar Aponeurosis (Fig. 7). These changes occurred in all volunteers. Although torque changed the internal arrangement, a consistent pattern could not be discerned (Fig. 8).

\section{Discussion}

The sole of the foot is the interface between the body and the ground during locomotion. It sustains the loading of the body and the forces of propulsion. Moreover, it helps to damp the impact forces and protects vulnerable structures. In general, compression is the largest force to which the sole is exposed. There are indications that the basic pattern of the sole of adipose tissue pervaded by septae of connective tissue, is important to withstand the compressive forces ${ }^{6}$. This pattern is present on a macro- as well as a microscopic scale. In this context, we would like to point to the fat bodies in the metatarsal region that protect the vessels and nerves of the digits from injury against the deep structures of the foot (e.g. the deep transverse metatarsal ligament).

Besides handling of the compressive force, the sole has to be pliable to be able to adapt to the relief of the ground for optimal contact on one hand and on the other hand be sufficiently firm to stabilize the sole against shear and torque. The internal structure should reflect these functions. Imagine a sole made up of adipose tissue, but without the connective 


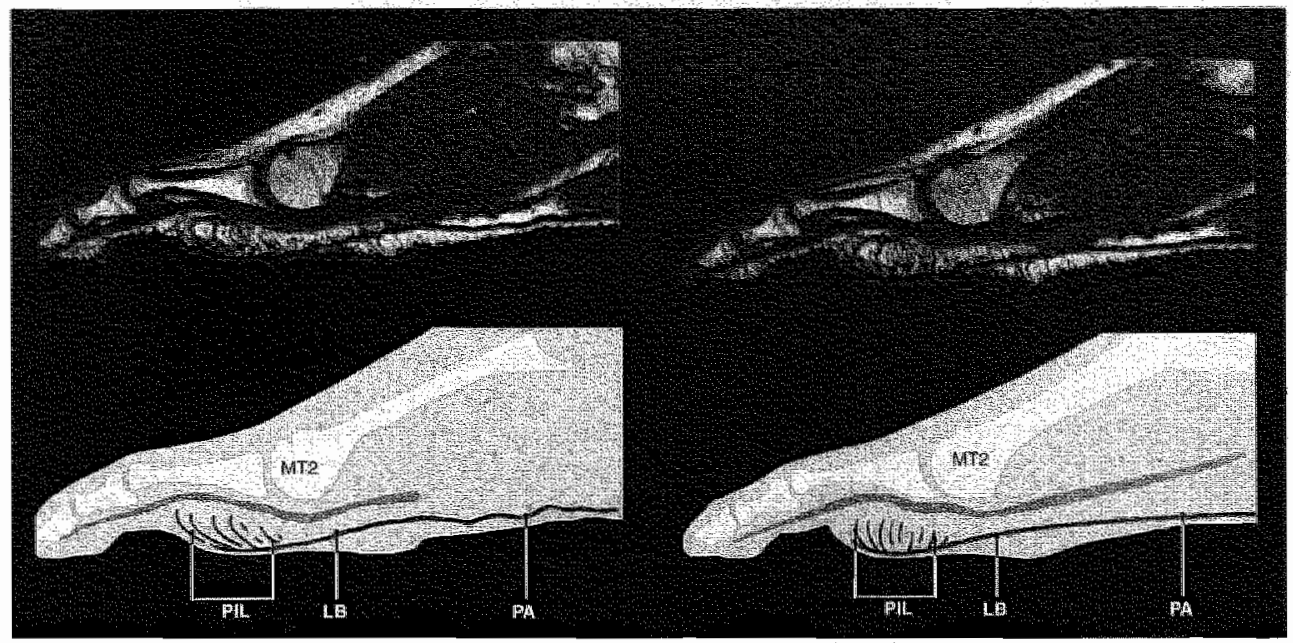

A

B

Fig. 7 : The effect of longitudinal shear on the sole of the forafoot at the second matatarsal head (MT 2 ). In

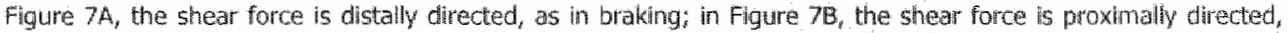
as in propulsion. The Planar Aponeurosis (PA) and the Longhudinal Band (LB) tur from buckled (Fig. $7 A$ ) into taut structures (Fig. 7B). The septae of the plantar Interdigital Ligament (PLL) redinect from: prodominatly oblique to vertical. The mass of the sale of the forefoot is positioned more distaly (Fig. 76 ).

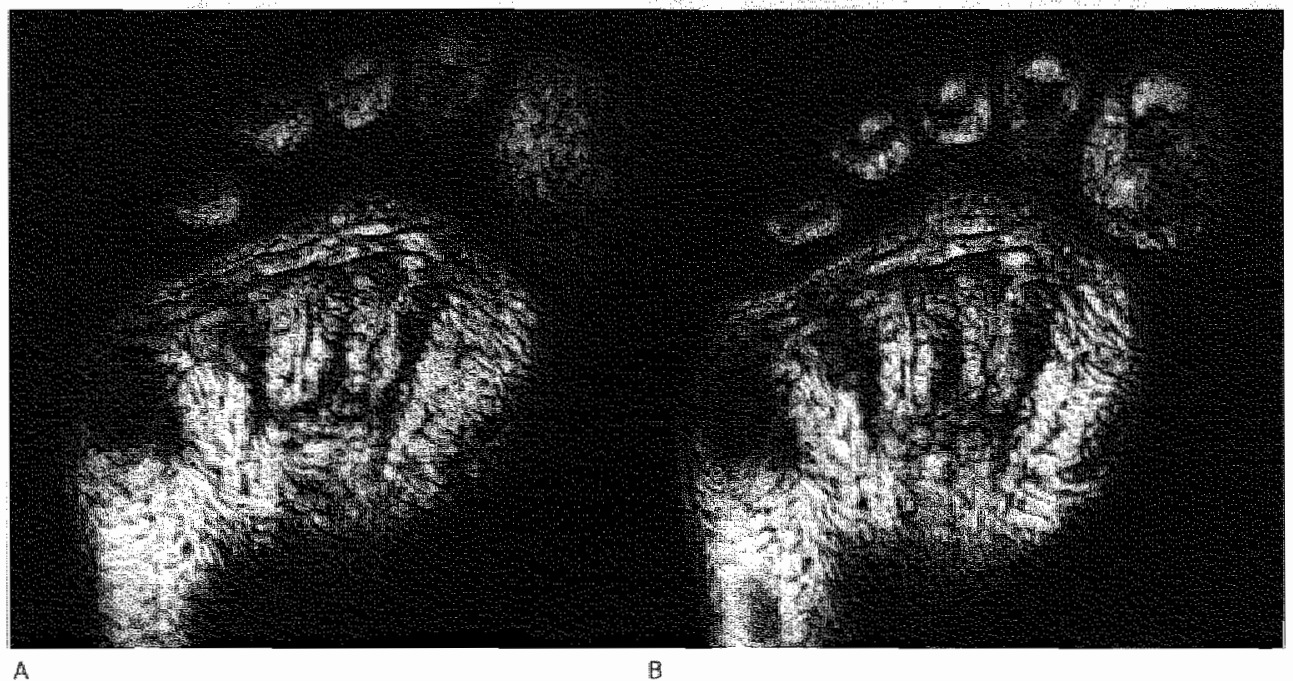

Fig. 8 : The efect of clockwise (A) and ant-clockwse (B) torque on the sole of the forefoot. Although the int arnal arrangement of the Piantar Interdigital Ligament changed, consistent pattem could not be discenmed.

tissue sheets. Although the sole would well adapt to irregularities on the ground, one would slip on it, because shear is not restricted. Imagine a sole made up of adipose tissue that is boxed in by vertical fibrous septae connecting the skin to the deep structures of the foot. The pliability does not necessarily diminish, and the fibrous septae would limit the translation of the skin relative to the deeper structures of the foot due to the shear and torque of locomotion. Imagine the septated sole (of the same thickness) with the addition of a fibrous 'inlay' within the subcutis in such a way that it connects to the skin by many short septae on one side and to the deeper layers of the foot by longer fibrous septae on the other side. This 
configuration would add to the stability of the sole and probably more important, local forces would be distributed regionally more effectively, before it seizes on the longer deep septae. The Subcutaneous Fibrous Layer and the Superficial Stratum of the Plantar Aponeurosis potentially are such structures and it seems reasonable to say that they help to stabilize the sole of the foot. The fixation of this inlay' to the dorsal fascia of the foot at the lateral and medial foot border will add to the stability of the sole.

The Superficial and Deep Stratum of the Plantar Aponeurosis, and the Plantar Interdigital Ligament are said to stabilize the sole in the longitudinal direction.. MRI could visualize this (Fig. 7) . During the propulsion phase, the metatarsal bones slide proximally on the sole by a proximally directed shear (propulsion) force. The skin and the sole of the forefoot are tethered by the Superficial Stratum. Moreover, as the Deep Stratum (indirectly) connects with the toes, dorsiflexion at the metatarsophalangeal joint will enhance the tension in the Deep Stratum. Thus, both the Superficial and the Deep Stratum limit this sliding, as they are pulled taut to transmit the shear force through the central part of the Plantar Aponeurosis to the calcaneal bone. The MRI simulations showed the changes in the Plantar Aponeurosis and the Longitudinal Bands and the shift of the metatarsals on the sole (Fig. 7).

During braking, the metatarsal bones slide anteriorly on the sole by an anteriorly directed force. As previously reported, a small anteriorly directed shear force induced the metatarsals to shift anteriorly about $5 \mathrm{~mm}$ on average ${ }^{14}$. Because the Plantar Interdigital Ligament ties the skin to the bases of the proximal phalanges, the ligament limits the anterior shift during braking. At the same time, the Plantar Interdigital Ligament helps to pull the toes firmly to the ground, which is important for the transfer deceleration. The MRI simulations showed the redirection of the Plantar Interdigital Ligament from a predominantly vertical to oblique (Fig. 7).

Bojsen-Moller et al. also illlustrated the foregoing ${ }^{15}$. In neutral position of the toes, the sole of the forefoot is a soft pliable structure. The hyperextension and abduction of the toes, as in push-off and toe-off, turn the pliable solle into a firm structure through tension of the Deep Stratum and the Plantar Interdigitall Ligament.

During heel-off to toe-off, the sole of the forefoot is exposed to lateral shear and torque. Considering the orientation and the connections of the Plantar Interdigital Ligament as visualized by MRI, the ligament also seems to be able to stabilize the sole against these forces. The firm connection of the transversely oriented ligaments with the Longitudinal Bands, the Superficial Stratum of the Plantar Aponeurosis, its fixation to the toes and the connection with the Superficial Fibrous Layer laterally, prevent the sole to shift sideways on torque or a shear force that is directed sideways. Forces in the Plantar Interdigital Ligament can be diverted along the lateral foot border though its connection with the Subcutaneous Fibrous Layer at the level of the fifth metatarsal head (Figs. 2A and 4). Although MRI visualized differences in the internal arrangement of connective tissue on provocation with torque, we could not discern a consistent pattern.

The use of terms such as bands, sheets, ligaments or tracts suggested the presence of dense and sharply delineated structures. However, these structures merely are reinforcements of the mesh of connective tissue in the direction of the prevailing loading. The only exception to this was the central part of the Plantar Aponeurosis before its division.

In summary, the current descriptions based on dissection of the internal architecture of the sole of the foot largely corresponded with the findings on MRI. In our view, the most important differences were the presence of the Subcutaneous Fibrous Layer beyond the heel region and its connections in the forefoot with the Superficial Stratum of the Plantar Aponeurosis and the Plantar Interdigital Ligament at the lateral foot border. These additions to the current knowledge of anatomy help to explain the function of the sole of the foot. 


\section{Acknowledgements}

We thank Rogier Trompert for his contribution to the illustrations.

\section{References}

1. Blechschmidt E: The structure of the calcaneal padding. Foot Ankle $1982 ; 2: 260-283$

2. Bojsen-Moller $F$ and Flagstad KE: Plantar aponeurosis and internal architecture of the ball of the foot. J Anat 1976; 121: 599-611.

3. Buschmann WR, Jahss $\mathrm{MH}_{\text {, }}$ Kummer $\mathrm{F}$, et al.. Histology and histomorphometric analysis of the normal and atrophic heel fat pad. Foot Ankle Int 1995; 16: 254-258.

4. Cralley $\mathrm{JC}_{\mathrm{n}}$ Schuberth IM and Fitch $\mathrm{KL}$ : The deep band of the planta: aponeurosis of the human foot. Anat Anz 1982; 152: $189-197$.

5. Hedrick MR: The plantar aponeurosis. Foot Ankle Int 1996;17:646-649.

6. Jahss $\mathrm{MH}_{3}$, Michelson $\mathrm{JD}$, Desal $\mathrm{P}_{\text {, }}$ et al: Investigations into the fat pads of the sole of the foot: anatomy and histology. Foot Ankle 1992; 13: 233-242.

7. Mitchell IR, Meyer C and Krueger WA: Deep fascia of the foot. Anatomical and clinical considerations. J Alm Podiatr Med Assoc 1991; 81: 373-378.

8. Saraffian SK: Anatomy of the Foot and Ankle. second. Philadelphia, Lippincot J.B. 1993.

9. Williams PL and Warwick R: Gray's anatomy. 36 th. Edinburgh, Churchill Livingstone. 1980.

10. Kimani JK: The structural and functional organization of the connective tissue on the human foot with reference to the histomorphology of the elastic fibre system. Acta Morphol. Neerl.-Scand. 1984; 22: 312-323.

11. Saraffian SK: Functional anatomy of the foot and ankle. In Anatomy of the Foot and Ankle, edited by Saraffian SK. second.. Philadelphia; Lippincot J.B. 1993. 474-602.

12. Delland JT, Lee K.T, Sobell $M_{x}$ et al.: Anatomy of the plantar plate and its attachments in the lesser metatarsal phalangeal joint. Foot Ankle Int 1995; 16:480-486.

13. Johnston RB, 3rd, Smith $J$ and Daniels $T$ : The plantar plate of the lesser tores: an anatomical study in human cadavers. Foot Ankle Int 1994: 15: 276-282.

14. Wejers $\mathrm{RE}$, wan Mameren $\mathrm{H}$, Walenkamp $\mathrm{GH}_{\text {, }}$ et al.: Changes of the soft tissue of the forefoot during loading: a volumetric study. Foot 2003; 13:70-75.

15. Bojsen-Moller $F$ and Lamoreux $L$ : Significance of free-dorsiflexion of the toes in walking. Acta Orthop Scand 1979; $50: 471 \times 479$. 


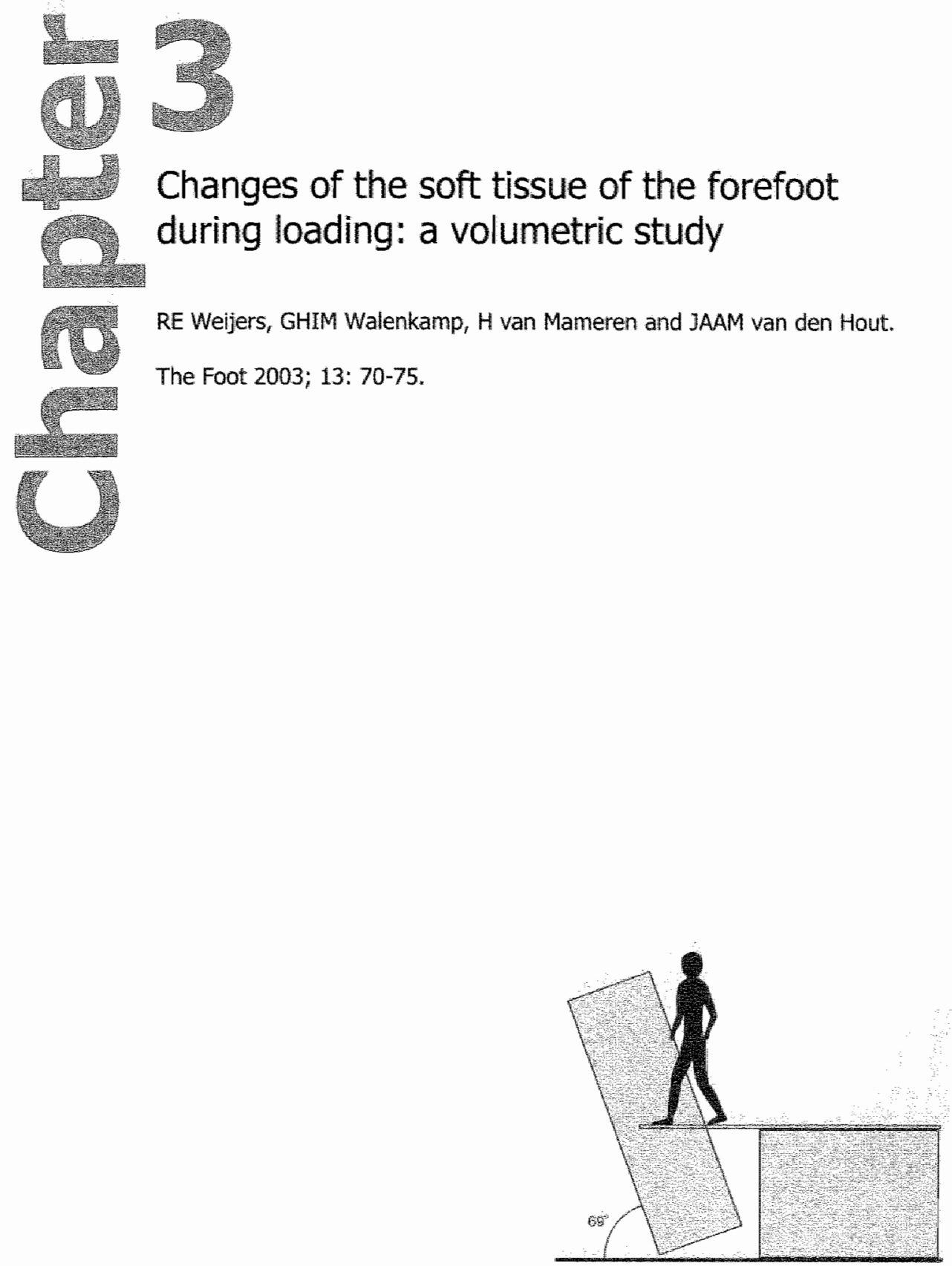




\section{Abstract}

Background: Although complaints of the forefoot are frequent, little is known about the changes in the soft tissue of the forefoot that take place during loading.

Objective: To study the volume changes of the soft tissue of the forefoot in reaction to loading in normal volunteers and to compare it to the described changes in the heel pad.

Method: With computed tomography (CT), the volume distribution of the soft tissue of the right forefoot of 11 healthy volunteers was examined in unloaded and in loaded posture.

Results. The decrease of the overall volume of the forefoot ( $4.8 \%$ ) in the loaded posture was located on the plantar side, A small ncrease in volume $(1.6 \%)$ occurred on the dorsal side of the forefoot at the metatarsal (MT) level.

Conclusions: The changes in soft tissue volume distribution in the forefoot by loading resemble observations in the heel region. The increase in volume on the dorsal side is unique for the forefoot and likely reflects a dorsal shift of soft tissue in the intermetatarsal spaces. The decrease in the overall volume was due to compression of plantar venous structures. Both observations may have an important function in shock absorption. In normal volunteers, the volume differences are so prominent that the described procedure may find dinical use in early identification of tissue damage. 


\section{Introduction}

Various conditions are accompanied by soft tissue changes of the foot sole, for instance diabetes mellitus ${ }^{1,2}$, rheumatoid arthritis ${ }^{3}$, atrophy ${ }^{4}$ or scarring. These changes are related to symptoms such as foot pain and dysfunction ${ }^{4-8}$. Detailled information about the function of the sole of the foot influence the type of therapy ${ }^{9,10}$.

During a gait cycle, the foot bears the weight of the body, adjusts itself to the surface, and it dampens the ground reaction forces before transmitting it to the rest of the body. This demands continuous adaptation of the skeleton and soft tissue of the foot.

The dampening properties of the sole of the foot are influenced by the size, location and integrity of the septa that form the chambered structure of the sole, as well as the fluid flow of the fat ${ }^{11}$ and the constituents of fat itself ${ }^{12}$. In this context, the potential hydromechanical dampening properties of the venous plexus in the sole are mentioned only once ${ }^{13}$. Most previous studies on the mechanical properties of the foot involve the heel pad. The vertical load of the calcaneus compresses the centre of the sole ${ }^{14}$ and displaces the soft tissue to the lateral and medial foot border ${ }^{15,16}$. Both atrophy and stiffening of the padding of the heel are related to higher plantar peak pressure ${ }^{9,17}$, a recognised risk factor in the development of complications, such as foot ulceration in diabetes mellitus ${ }^{18}$.

We assume that adaptation in the forefoot differs from that in the heel. In the forefoot, the soft tissue is supported by flexibly connected skelletal structures, while in the heel the calcaneus is rigid. Moreover, the septal anatomy of the ball of the foot ${ }^{16,19}$ differs from that of the heel pad ${ }^{4} 16$.

In view of the foregoing, we studied the volume changes of the soft tissue of the forefoot in reaction to loading in normal volunteers with computed tomography (CT) and compared it to the described changes in the heel pad. These data may lead to a better understanding of the relation between frequently occurring pathologicall soft tissue changes in the foot and clinical symptoms.

\section{Material and methods}

Eleven volunteers were included: six males and five females (mean 35 years, range 19-51). They had all given informed consent. All volunteers were healthy without complaints of their feet or a history of previous trauma or surgery.

The right foot of the volunteers was scanned while they were standing barefooted and upright on the tabletop of a spiral CT scanner (Elscint Twin RTS, Old Delft, Delft, The Netherlands). With maximal tilt of the gantry $\left(21.2^{\circ}\right)$ the geometry of the CT scanner still prevented a normal one-legged stance. To keep their balance during loading the volunteers had to place their left foot behind the right on the tabletop (Fig. 1). A pressure measuring inlay (Pedar system, Novel GmbH, Munich, Germany) was fixed to the tabletop to check for a normal loading pattern. Also, a radiopaque marker was fixed to the tabletop and served as a reference point to analyse movements of bones relative to the tabletop.

The right foot was positioned with the axis of the second metatarsal (MT) parallel to the lateral border of the tabletop. The first tarso-metatarsal joint of the right foot (TMT 1) was located by palpation and the skin was marked. The spiral scan of the forefoot covered the area from TMT 1 to the most distal interdigital web. To give visual feed back on the distribution of the plantar pressure, the volunteers could see the monitor of the Pedar system while standing on the tabletop. 


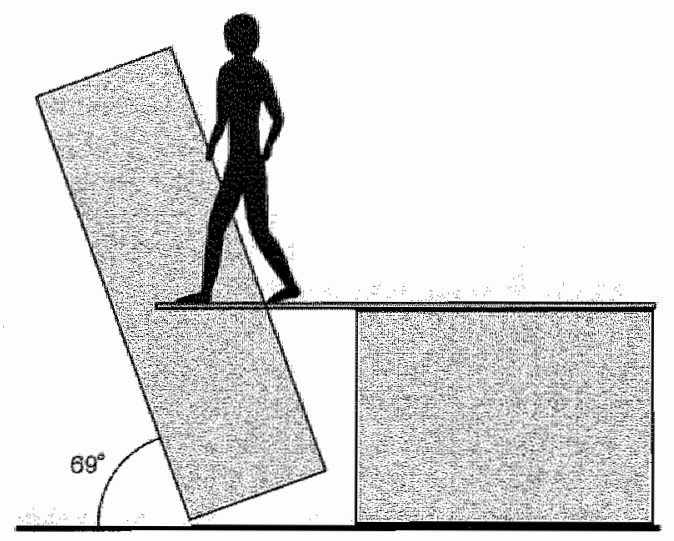

Fig. 1: Schematic drawing of the posture of the wolunteer on the tabletop of the CT scanner during loading.

The right forefoot was scanned in unloaded and in loaded posture. During the unlloaded posture the volunteer placed the body weight on the left foot and gently laid the right foot on the pressure measuring device. The first scan was started when the pressure measuring device contacted both the ball and the heel of the foot and the pressure did not exceed $2 \mathrm{~N} / \mathrm{cm}^{2}$ at any place for $15 \mathrm{~s}$. Next, the volunteers shifted the body weight to the right foot as much as possible. At least $3 \mathrm{~min}$ preparation time were needed before the second scan was started. The second scan was made only when the maximum pressure under the forefoot and the maximum pressure under the heel were equal for $15 \mathrm{~s}$. Between the two scans the sole of the foot did not lose contact with the tabletop. We used the following scan parameters: $120 \mathrm{kV}, 67 \mathrm{mAs}$, pitch 1.5 , field of view $203 \mathrm{~mm}, 512^{2}$ matrix, $1.1 \mathrm{~mm}$ slice thickness and bone allgorithm. The scans of the first two volunteers were reconstructed using $0.6 \mathrm{~mm}$ increment; afterwards we used $1.1 \mathrm{~mm}$ increment.

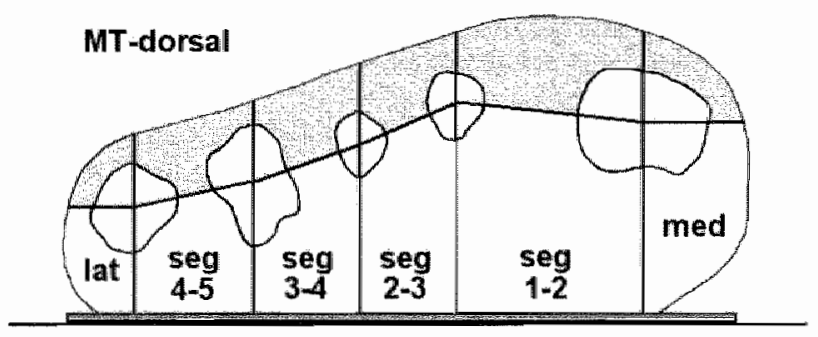

\section{MT-plantar}

Fig. 2: schematic drawing of a segmented CT-image at a metatarsal level. Wertical lines through the centroids define the segments: two foot border segments (med and lat) and four central segments (seg 1-2 up to seg 4.5). Only in the MT'-region, lines connecting adjoining centroids and two horizontal lines separate plantar and dorsal segments. The MT-dorsal region is grey, the MT -plantar white. Note the pressure measuring inlay under the foot (dark grey).

To calculate the distribution of the soft tissue in the forefoot, we first processed the images. On each image the centroid of the MTs and proximal phalanges were marked, a centroid being the point with coordinates that are the average of the $x$ - and $y$-coordinates of all the pixels of that bone. Through the centroids vertical lines were drawn separating six segments: 
two foot border segments (the medial and the lateral segment) and four central segments (segments 1-2 up to 4-5). Only in the MT region (see later) each segment was further divided into a plantar and a dorsal side by four lines connecting the centroids of adjoining bones and two horizontal lines through the centroids of the first and fifth MT bones (Fig. 2). In all the sectors of each image the pixels were counted. We used the Easyscil 4.2.1 software package (Philips, Best, The Netherlands) for this semi-automated analysis.

The sectors of the images were grouped into regions. The metatarso-phalangeal (MTP) region is made up of the phalangeal region and the MT region (Fig. 3). Only in the MT region a plantar (MT-plantar) and a dorsal side (MT-dorsal) were distinguished (Fig. 4). The borders of these regions were defined by:

- MTP region: the proximal border was the image through TMT 1. Images through the four interdigital webs gave the distal border.

- Phalangeal region: the distal border was the same as the distal border of the MTP region. The proximal border is positioned halfway the images through adjacent MTP joint spaces (Fig. 5).

- MT region: the distal and proximal border were the same as the proximal border of the phalangeal region and the proximal border of the MTP region.

To determine these borders specific images are used:

- interdigital web: the most distal image that still showed soft tissue between two adjacent toes,

- MTP joint space: the most distal image that showed the cortex of both the MT head and base of the proximal phalanx,

- TMT 1: the image that showed the largest part of the joint space of TMT 1.

The volumes of the segments were calculated by multiplying the total number of their pixels by the pixel volume. The total number of pixels is the sum of all the pixels in all images that were assigned to that segment. The total volumes of the regions were calculated by the summation of the volumes of their segments. This procedure was carried out for both the loaded and the unloaded posture. The volume differences were expressed as the percentage of the volume in the unloaded posture. The differences were statistically analysed with the two-tailed one sample $t$-test using SPSS 10.0.0 for Windows software (SPSS inc, Chicago, IL, USA).

By definition the calculations of the volumes depend on position of the bones. Therefore, we analysed the shift in the anterior-posteriar direction of the MTs relative to the tabletop during loading. For each MT in both postures we determined the number of Images between the one with the reference marker fixed to the tabletop and the one with the MTP joint space. The differences were multiplied by the slice increment and expressed in millimetres.

In the phalangeal region we also calculated the length of its segments (defined as the differences in the distance between the proximal and distal border) in the loaded and in the unloaded posture. The number of slices included in the segments of the phalangeal region was multiplied by the slice increment for both postures; these results were subtracted and expressed in millimetres. Because of the partial volume effect of the $C T_{\text {, differences in }}$ distance of one slice thickness were ignored. 


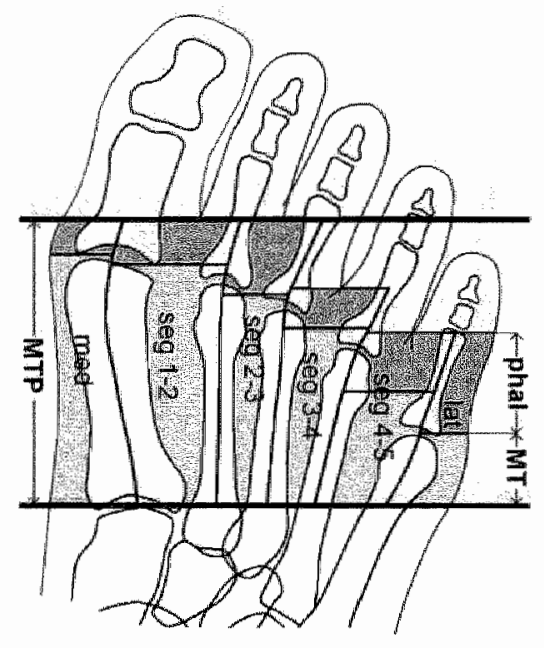

Fig. 3: Schematic drawing of the reglons and segments of the forefoot. Whith the scanmed wolume, marked by the two thick black 眇es, the proximal and distai borders of the metatarsophalangeal region (MTP), phalangeal region (phal) in dark grey, and netatarsal region (MT) in light grey are llustrated. The curved lines lengthwise represent the centroids of the metatarsals and proximal phalanges on successive images. They separate the segments: medial foot border segment (med), segments 1 -2 up to 4-5 (seg 1-5) and laterall fort border segrnen (lat). Mote: The positioning of the borders beween MT and phalangeal iregion is illustrated in detall in Figure 5 .

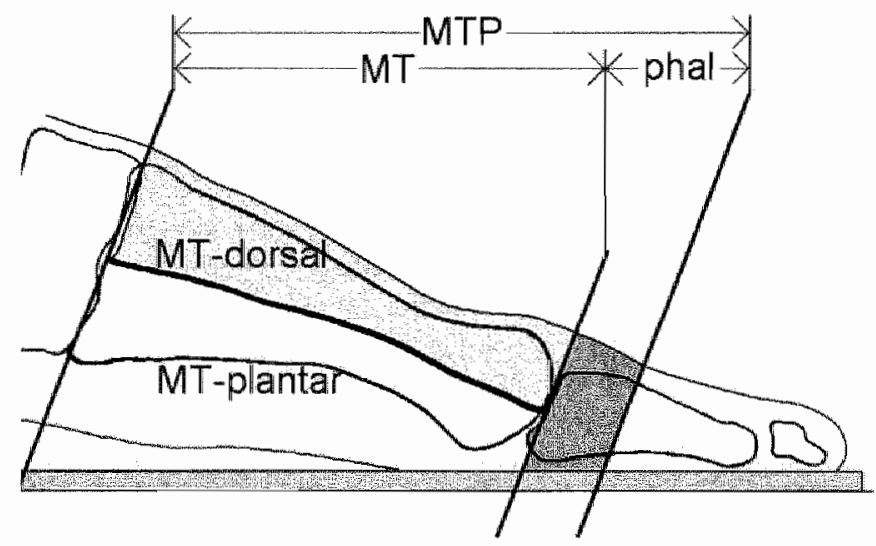

Fig. 4: Saghtial section though the centroids of bones of the grat toe. The bonders of the matatarso-phalangeal region (Mith), subdivide in the MT megion (MT) and phalangeal region (phal), are indicated by the tited lines. The cured line throgh the centroids of the first metaluasal on succersive images divides the metatarsall region in MT-dorsoll (light grey) and MT-plantar (whte). The phatangeal reghon (dark grey) is not divided. 


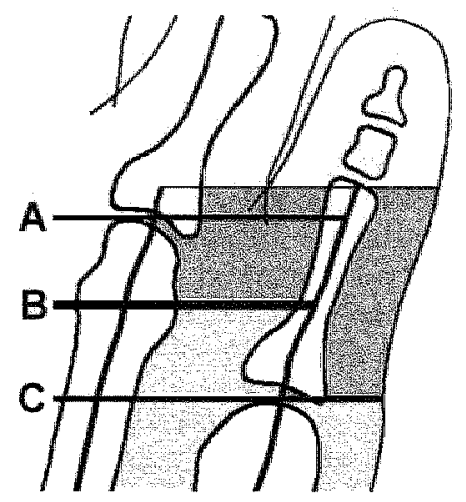

Fig. 5: In the segment 4-5, the border (ine B) between the MT region (inght grey) and the phalangeal regom (dark grey) conrelates with the CT image positioned halfway the image through the fourh (hine and tha fifh (ine C) metatarsowphalangeal joint. Nota: In the lateral segnent the border between the Mr wegion and the phatangea region correlates whth the image though the fifth metatarso-phalangeal jomt (tine $c$ ).

\section{Results}

For all regions and segments, Figure 6 summarises the mean volume differences. The total volume of the MTP region decreased by $4.8 \%$ on loading. All its segments contribute to this decrease (Fig. 6A). The loss of volume in the phalangeal region is more prominent than in the MT region (Fig. 6B). The decrease in the MT region is the result of a small increase in the MT-dorsal and a larger decrease in the MT-plantar (Fig. 6C).

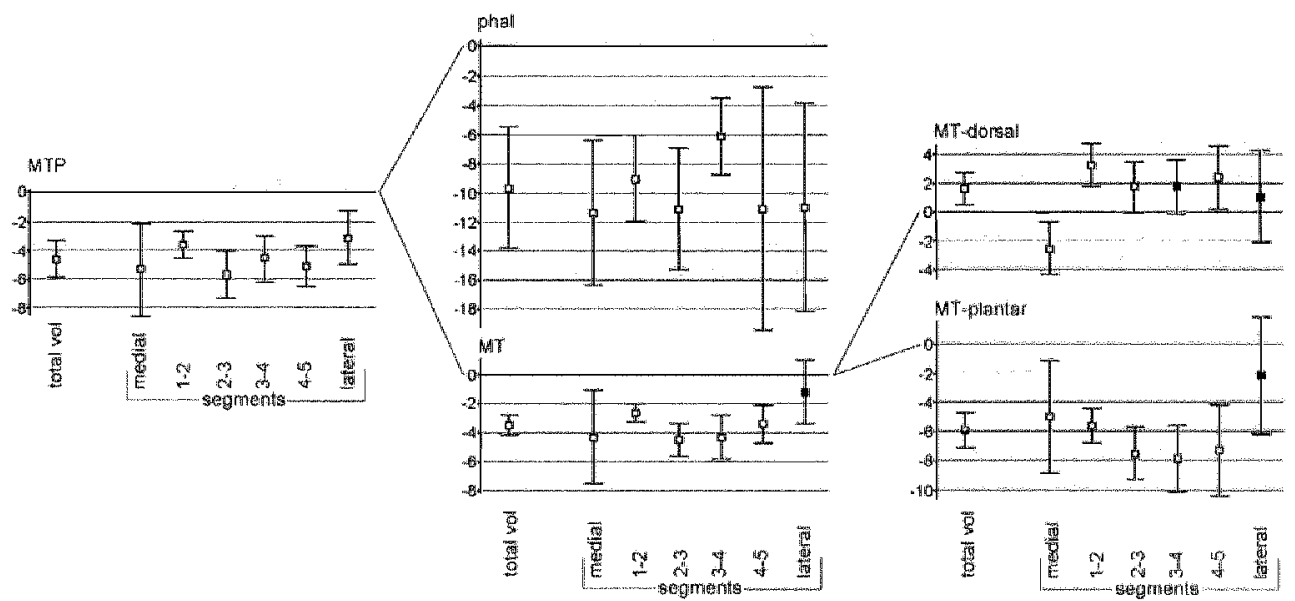

(A)

(B)

(C)

Fig. 6." The volume differances between unioaded and loaded posture are given as percentages of the unilouded posture. The resules of the total volume (total vol), medial foot border segment, segments 1.2 up to 4.5, and ateral foot border segments of (A) the metatarso phatangeal regron (MPP), of (B) phalangeal (phal) and MT region, and of (C) MT-dorsal and MT-plantar are given. The $95 \%$ conflence htervals and the mean wolume differences (squares) are given. A mean volume differmce that is not significant is marked by a closed square. 
The differences in the mean volumes are statistically significant $(P<0.05)$ except for segment $3-4$ of the MT-dorsal region and the lateral foot border segments of the MT, MT-dorsal and the MT-plantar regions. Most of the lateral and medial segments show a wider $95 \%$ confidence interwal than the other segments.

The mean volume differences in the lateral segment of the MTP, MT and MT-plantar regions seem to differ from those of the central segments. This was statistically tested by a paired ttest on data of the MTP, MT and MT-plantar regions: the average volume differences of all the central segments of each volunteer were calculated and paired to the volume changes of the lateral segment. This difference proved to be significant ( $P$ values respectively 0.049 , 0.014 and 0.011 ).

The medial segment of the MT-dorsal region shows a decrease while the other segments of this region show an increase. A paired $t$-test showed this difference to be significant $(P<0.001)$.

Table 1 summarises the shift of the MTP joint spaces in the antero-posterior direction during loading. In nine volunteers the MTP joint spaces moved forward during loading relative to the tabletop; in two volunteers no change was observed. The forward shift is more pronounced on the medial than on the lateral side.

Talble 1. Arterior shif during loading. The shitt (in mm) of the metaturso-phalangeal joint space in anteroposterior direction. Positive numbers indicate athift in the anterior drection.

\begin{tabular}{lc}
\hline & mean (min-max) \\
\hline MTP 1 & $6(1-11)$ \\
MTP 2 & $5(0-5)$ \\
MTP 3 & $4(0-8)$ \\
MTP 4 & $3(0-6)$ \\
MTP 5 & $3(0-6)$ \\
\hline
\end{tabular}

In the phalangeal region the length of its central segments did not change on average during loading. In 23 out of 34 segments the length was unchanged and 10 times a decrease was noted. Only once an increase in length occurred. In 10 out of 44 segments (four in segment 1-2 and 2-3 and two in segment 3-4), the length could not be calculated because the scan was ended before the interdigital webs were reached. These segments were excluded from analyses. This was also taken into account in the calculation of the statistics of the phalangeal region.

\section{Discussion}

Unlike gas, fluid and solids do not change their volume or density under physiological compression, they can only change shape. Therefore, we were surprised to find a decrease in the total volumes of all regions except the MT-dorsal. A shift of solid soft tissue out of the scanned volume has to be considered. The scanned volume included the interdigital webs distally, excluding a loss of volume distally. A lass proximally beyond the border of the MTP region was improbable because the loss was evenly distributed along the length of the segments. A shift of fluid out of the scanned volume offers a more plausible explanation: during loading, venous structures empty by compression. It agrees with both functional anatomy and the observation that the volume loss in the MT region is located on the plantar side. It is illustrated in Figure 7. In the sole of the foot an extensive mesh of venules without valves is present in the intradermall and subcutaneous tissue. Larger veins are situated 
centrally and contain valves in half the individuals ${ }^{20,21}$ "In the unloaded upright posture, the venous structures are filled. In standing or walking, the plantar pressures empty the venous structures ${ }^{9}$. Repetitive compression of the venous structures enthanices the venous outflow of the lower limb ${ }^{21}$. Considering the amount of blood involved, the venous structures of the sole can also function as a hydro-mechanical damper. The status of the venous plexus of the sole in various diseases affecting the foot and its relation to clinical symptoms needs further investigation.

Not all the segments in the MT-plantar region show similar volume loss. Both foot border segments show a smaller reduction in volume than the central segments do. It is most likely that in the foot border segments the volume reduction by venous compression is counteracted by an outward shift of solid soft tissue from the central segments. Although this cannot be proven from our data, this shift agrees with the model of loading of the heel region ${ }^{16,1.7}$. The foot border segments give a wider $95 \%$ confidence interval than the central segments. This agrees with the wide variation in the increase in width of the heel pad across normal individuals on loading ${ }^{15}$.

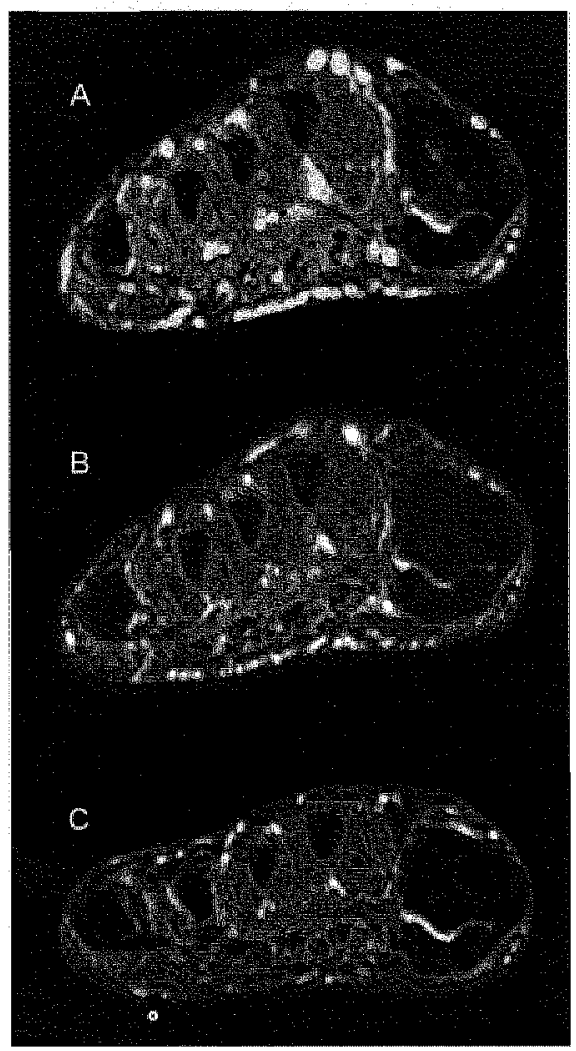

Figg. 7: Axial fat-suppressed Mr indages (STIR: TR/TE/TI: 1400/20/150 ms.) at the level of the sesamolds show diferent degrees of filling of the venous structures of the sole of the foot: A volunteer was scamned in supine position. In $(A)$ a toumiquet was phaced just below the knee, in (B) the toumiguet was removed and in (C) the sole of the fore foot was compressed by sandbag of two kg only.

The total volume of the MT-dorsal region increased $1.6 \%$ during loading. This increase could be explained by a shift of blood from the sole to the dorsum of the foot through the marginal veins. However, the 3 min elapsing between loading and measuring should be 
sufficient to re-establish the original venous blood volume in the MT-dorsal region. It seems that soft tissue shifts from the sole to the dorsal side of the foot. In the heel region tissue curls around the calcaneus ${ }^{16,17}$, but in the forefoot the volume increase of the dorsal segments is more pronounced centrally than in the foot border. Tissue seems to move from plantar to dorsal through the spaces between the MTs. Only the medial foot border segment showed a significant decrease in volume. We cannot explain this observation.

During walking and running, the loading force on the foot increases two to three times the body weight with a high initial loading rate ${ }^{7,16}$. Shock damping is proportional to the velocity of deformation. It is, therefore, conceivable, that the plunger-like movement of tissue through the space between the metatarsals during a loading cycle, will be important for shock damping in the forefoot. In this respect the forefoot differs from the heel.

In analogy to the heel pad, we expected the soft tissue of the ball of the foot to move outward during loading, giving an increase in length of the segments of the phalangeal region. Surprisingly, this happened only once. The MTP joints moved distally relative to the soft tissue of the sole. The expected spreading of the soft tissue towards the web spaces seemed to be counteracted by the anterior movement of the bones relative to the soft tissue. This, together with the venous compression, resulted in a $9.8 \%$ wolume loss of the total volume in the phalangeal region. We realise that this observation is probably an artifact due to a shear force caused by the posture of the volunteers on the tabletop. Nevertheless, it may have dinical importance: to facilitate these changes, the soft tissue of the ball of the foot must be very pliable. This pliability is called upon during gait: as the toes extend and abduct during push-off, the internal fibro-elastic architecture in the ball of the foot changes the ball from a soft into a tense and firm structure that anchors the skin to the deeper structures ${ }^{19}$. Change in tissue composition can disturb this delicate mechanism. For instance, in diabetes mellitus specific complications are related to stiffening of tissue ${ }^{1,2,9,22}$.

$C T$ is an outstanding method to evaluate bones in relation to soft tissue, but some caution in interpretation of the results is required:

- The geometry of the scanner precluded a full scan of the foot. The scan could include the entire first MT, but only part of the length of the other MTs (Fig. 3). Therefore, we also analysed the distribution of the volume changes along the shaft of the MTs. In general, we noted an even distribution lengthwise in the entirely included first metatarsal as well as the partly scanned other MTs. This justifies our study design and results.

- By definition, the measurement of the soft tissue volumes depended on the position of the osseous structures. During loading not only the soft tissue changes shape, but also the bones move relative to each other. This is the reason why we did not divide the phalangeal region into plantar and dorsal segments. The toes extend during loading and affect the positions of their centroids. It makes separate volume calculations of plantar and dorsal segments meaningless. In the ather regions the effect of movement of bones on the results cannot be quantified and possibly affects the results of the smaller segments. The effect will probably be minor in the larger volumes of the reglons.

We conclude that the changes in soft tissue volume distribution in the forefoot on loading resemble those observed in the heel region. The supposed dorsal shift of soft tissue in the intermetatarsal spaces is unique for the forefoot and may have an important function in shock absorption. In normal volunteers the volume differences are so prominent that the described procedure may find clinical use in detecting damaged soft tissue. 


\section{Acknowledgements}

We thank Jos wan Engelshowen, Gerrit Kemerink and Jan Wilmink for their Kelpful suggestions and review of the manuscript, Mark Geerlings for his technical assistance, Alfons Kessels for his statistical advice, and the physicians for their support.

\section{References}

1. Brownlee M: Gycation products and the pathogenesis of diabetic complications. Diabetes Care 1992; 15: 18351843.

2. Kao PF, Davis Bl and Hardy PA: Characterizaton of the calcaneal fat pad in diabetic and nonadiabetic patients using magnetic resonance imaging. Magn Reson Imaging 1999; 17:851-857.

3. Lichniak JE: The heel in systemic disease. Clin Podiatr Med Surg 1990; 7: 225-241.

4. Jahss MH, Michelson JD, Desal $\mathrm{P}$, et al.: Investigations into the fat pads of thes sole of the foot: andtomy and histology, Foot Ankle 1992; 13:233-242.

5. Jorgensen U: Achillodynia and loss of heel pad shock absorbency. Am J Sports Med 1985; 13: 128-132.

6. Prichasuk S: The heel pad in plantar heel pain. J Bone Joint Surg Br 1994; 76: 140-142.

7. Rome K: Mechanical properties of the heel pad: current theory and review of the literature. The Foot $1998 ; 8$ : 179-185.

8. Tsai $\mathrm{WC}_{n}$ Wang $\mathrm{CL}_{\text {, Hsu }} \mathrm{TC}$, et al.: The mechanical properties of the heel pad in unilateral plantar herel pair syndrome. Foot Ankle Int 1999; 20: 663-668.

9. Cavanagh PR, Utbrecht JS and Caputo GM: Biomechanicall aspects of diabetic foot disease: aetiology, treatment, and prevention. Diabet Med 1996; 13 Suppl 1: $517-22$.

10. Gould JS" Metatarsalgla. Orthop Clin North Am 1989; 20:" 553-562.

11. Oomens CW, wan Campen DH and Grootenboer HJ: In witro compression of a soft tissue laver on a rigid foundation. J Blomech 1987; 20: 923-935.

12. Buschmann WR, Jahss $M H$, Kummer $F$, et al.: Histology and histomorphometric analysis of the normal and atrophic heel fat pad. Foot Ankle Int 1995; 16: 254-258.

13. Bojsen-Moller $F$ and Flagstad KE: The plantar soft tissue: Fundional anatomy and dinical applications, in Disorders of the foot and ankle, Medical and Surgical management, ed by Jahlns MH. Saunders, WB, Philadelphia; $1991.532-540$.

14. De Clerca D, Aerts $P$ and Kumnen M: The mechanical characteristics of the human heel pad during foot strike in running: an in: wivo coneradiographic study. ] Biomech 1994; 27: 1213-1222.

15. Fuller $\mathbf{E}$ and Hogge JD: Measurement of the expansion of the calcaneal fat pad upon weightbearing. I Am Podiatr Med Assoc 1998; 88: 12-16.

16. Saraffian SK: in Anatiomy of the foot and Ankle, ed by Lippincott JB, Phlladelphia; 1993. 590-596.

17. Jahss MH, Kummer F and Micheison JD: Inwestigations into the fat pads of the sole of the foot: hieel pressure studies. Foot Ankde 1992; 13:227-232.

18. Boulton $\mathrm{AJ}$ : The pathogenesis of diabetic foot problems: an ovewiew. Diabet Med 1996; 13 Suppl 1: 5.12-16.

19. Bojsen-Moller F and Flagstad KE: Plantar aponeurosis and internal architecture of the ball of the foot. I Anat $1976 ; 121: 599-611$.

20. Saraffian SK: in Anatomy of the Foot and Ankle, ed by Lippincot 1,B , Philadelphia; 1993. 349-353.

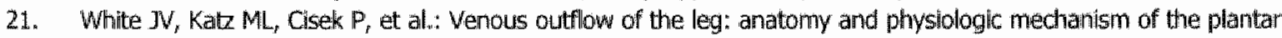
venous plexus. J Vasc Surg 1996; 24: 819-824.

22. Birke JA, Firanks BD and Foto JG: First ray joint limitation, pressure, and ulceration of the first metatarsal head in diabetes mellitus. Foot Ankle Int 1995; 16: 277-284. 


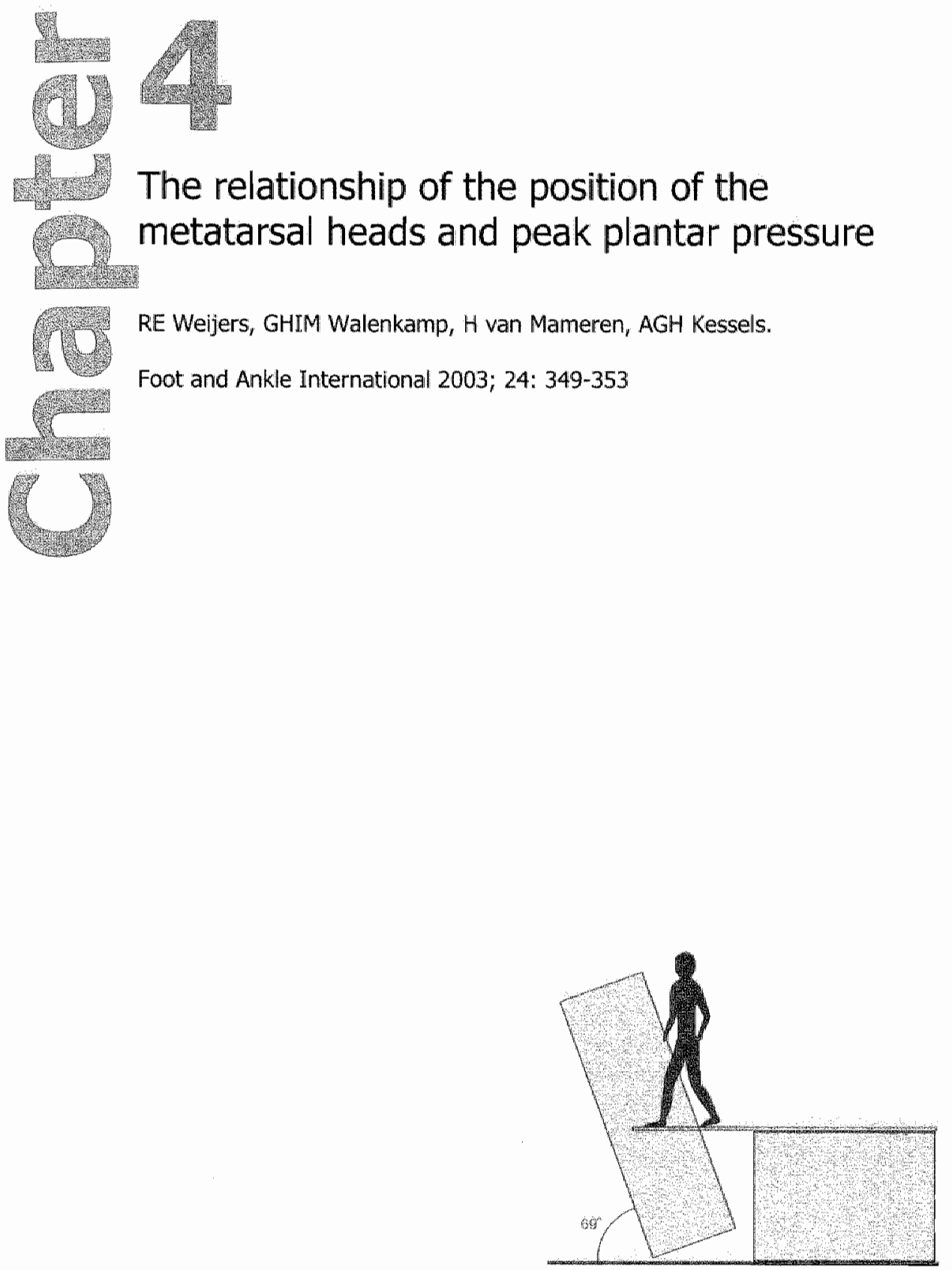




\section{Abstract}

We tested the premise that peak plantar pressure is located directly under the bony prominences in the forefoot reglon. The right foot of ten standing volunteers was examined in three different postures by a CT-scaniner. The plantar pressure distribution was simultaneously recorded. The position of the metatarsal heads and the sesamoids could be related to the corresponding local peak plantar pressures. The metatarsal heads 1,4 , and 5 had a significantly different position than the local peak plantar pressures. The average difference in distance between the position of the metatarsal heads and the peak plantar pressure showed a significant correlation: on the medial side the head was located more distally to the local peak plantar pressure, on the lateral side more proximally. The findings suggest that normal plantar soft tissue is able to deflect a load. The observations might improve insight into the function of the normal forefoot and might direct further research on the pathological forefoot and on the design of footwear: 


\section{Introduction}

In all studies of plantar pressure, soft tissue and bone, the data are gathered and interpreted under an intuitive premise. It is that during loading the most plantar parts of the bony prominences (the metatarsal heads, sesamoids and uber calcanel) give the largest local ground reactive force. Therefore the local soft tissue of the sole should become more compressed than its environment. Although non-surgical therapy ${ }^{1-10}$ and surgical interventions ${ }^{11}$ are based on this premise, the premise has not been studied. The purpose of this study is to test this premise by relating the position of the bony prominences of the forefoot to peak plantar pressures in normal volunteers. We simultaneously used a spiral CT scanner and a pressure measuring device. We hypothesize that the position of local peak pressure coincided with the position of the metatarsal heads (MTH).

\section{Materials and methods}

Our ten volunteers included five males and five females (mean 34 years, range 19-51). The study was approved by our Institutional Review Board. All volunteers gave informed consent. They were healthy subjects, without complaints of their feet, callus, deformation, or a history of previous trauma or surgery.

On the tabletop of a CT scanner (Elscint, Oldelft, Delft, the Netherlands) with the gantry maximally tilted $\left(21.2^{\circ}\right)$ a perspex plank was fixed. A pressure measuring inlay (the Pedar system, Novel GmbH, Munich, Germany) was mounted on the plank. In the plank holes of $1 \mathrm{~mm}$ diameter were vertically drilled forming an orthogonal coordinate system. The mediallateral and antero-posterior directions were defined as respectively the $\mathrm{x}$ and $\mathrm{y}$ direction. The pressure measuring device was calibrated immediately prior to each experiment with the 'Trublu' calibration device (the Pedar system, Novel GmbH, Munich, Germany).

During the experiment the volunteers were standing upright on bare feet, with the right foot in front of the left, on the tabletop of a spiral CT scanner (Fig. 1). The right foot was positioned on the inlay with the axis of the second metatarsal bone parallel to the $y$-axis. For each volunteer three consecutive scans of the right forefoot were made in three different postures. Between the scans the sole of the foot did not lose contact with the tabletop. During the fifteen seconds prior to the start of each scan we recorded the pressure distribution. In the first posture the volunteers laaded the right foot maximally: the loaded posture. The scan was started when the maximum pressure in the region of the ball equalled the maximum pressure under the heel region for a period of fifteen seconds. Than the volunteers loaded the forefoot as much as possible while the heel remained in contact with the inlay: the heel-off posture. The second scan was started when the recorded maximum pressure over the forefoot was invariable and the pressure over the heel was only about $2 \mathrm{~N} / \mathrm{cm}^{2}$ for a period of fifteen seconds. On the last scan the volunteers gave maximum pressure on the forefoot while extending the foot as much as possible: the toe-off posture. To give the patient visual feed back on the plantar pressure, the volunteers could see the monitor of the Pedar system while standing on the tabletop.

The spiral scan of the forefoot ranged from the first tarso-metatarsal joint to the most distal interdigital web. We used a $21.2^{\circ}$ tilted frontal plane, $120 \mathrm{kV}, 67$ mAs, a pitch of 1.5 , a field of view of $203 \mathrm{~mm}$, a $512^{2}$ matrix, a $1.1 \mathrm{~mm}$ slice thickness and a bone algorithm.

The inlay, European shoe size 47 , measured normall interface stress with ninety-nine sensors. The average size of the sensors over the forefoot region was $1.8 \mathrm{~cm}^{2}$. The accuracy of measurement was better than $5 \%$. 


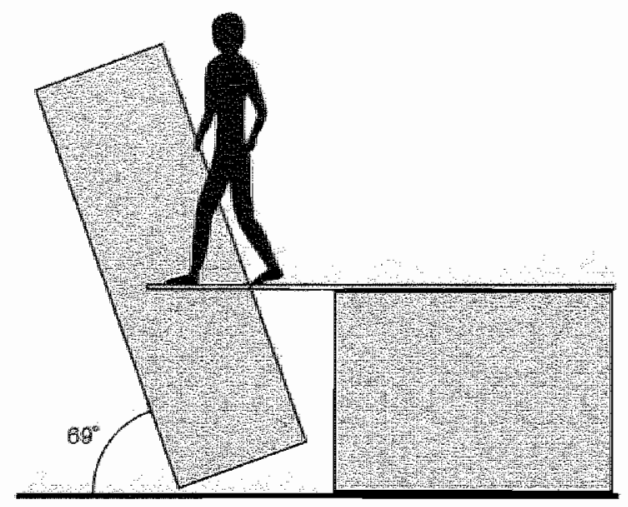

Fig. 1: Schematic drawing of the posture of the volunteers on the tabletop of the CT scanner during loading.

To link the position of the peak plantar pressure to bony prominences, the position of both the corners of the pressure elements and the bones were expressed as coordinates in the coordinate system in the perspex plank.

The coordinates of the corners of the pressure sensitive elements of the inlay were directly measured on the plank with the coordinate system (Fig. 2A). To obtain pressure distribution on a finer grid, each pressure measuring element was interpolated into nine smaller areas of approximately 4.2 and $5.3 \mathrm{~mm}$ in respectively the $x$ and $y$ direction (Fig. 2B). The coordinates of the corners of the interpolated areas and the corresponding pressure values were calculated (Fig. 2C).

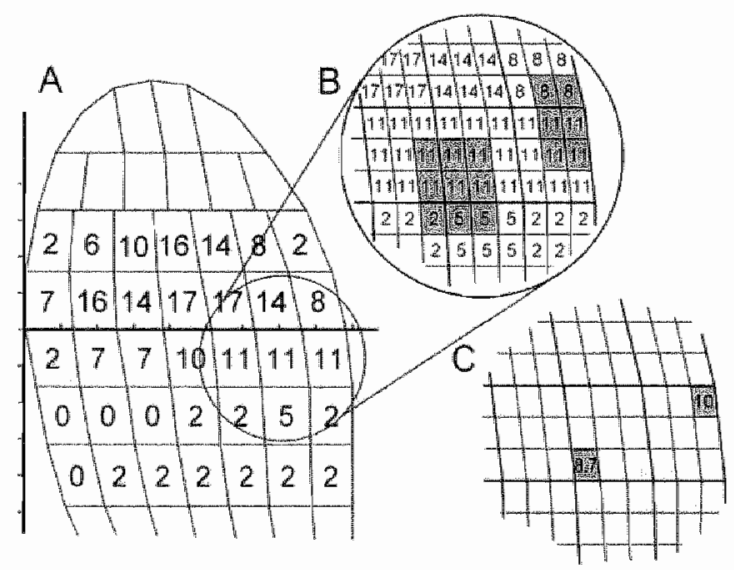

Fig. 2: Illustration of the process of interpalation of the pressure distribution. Ari example of the pressture distribution of one of the voluntears ([numbers] $=\mathbb{N} / \mathrm{cm}^{2}$ ) is shown (A). The coordinate system as drilled in the underlying plank is also depicted. Each border of a pressure measuring element was divided into three equal parts, yleiding nine areas. To each area the pressure reading of the originating element was given $(B)$. The pressure of an intepolated area (grey) was calculated as the average of the concerning area and its eight neighboring areas, excepted for arears at the border of the inlay where five neighboring areas were used (C). The resulting matrix is shown in Figure 3.

The position of the metatarsal heads (MTH) and sesamoids was defined as the point on its cortical surface where the soft tissue thickness of the sole underneath was minimal. The positions of the metatarsals and the sesamoids were determined using 3D multi-planar 
reformatting on a workstation (EasyScll 4.2.1, Philips, Best. The Netherlands) and inarked in the scanned volume. These marked positions were vertically projected on the (horizontal) plane of the plank and read out on the coordinate system. These coordinates were noted for each patient, posture, sesamoid, and MTH. This procedure was carried out twice. The average of both measurements was finally used for coordinates. To estimate the reproducibility of this method, the distance between a position ilit the first and second measurement (defined as the square root of the sum of the squares of the differences of the $x$ and $y$ coordinates) was callculated. The mean distance between the first and second measurement was $0.52 \mathrm{~mm}$ (min-max: 0.0-1.74 mm; stdv: 0.32). The distance was less than $1 \mathrm{~mm}$ in $93 \%$ of the cases.

Knowing the coordinates of both the bony prominences and the areas, the positions of the MTHs and sesamoids were marked on the interpolated pressure distribution map (Fig. 3). To test the hypothesis that the position of local peak pressure coincided with the position of the MTH, the distance between the position of the MTH and 'the line of maximum pressure' was determined. The 'line of maximum pressure" was the line that connected all the areas with the maximal pressure in each column of the interpolated pressure distribution map (Fig. 3). The number of areas between this line and the position of the metatarsals and sesamoids was noted and multiplied by $5.3 \mathrm{~mm}$ (the length of an area in the $y$-direction). The null hypothesis was that the position between the bony prominences and the line of maximum pressure' coincided. It was tested two sided with the one-sample t-test for all bones and each posture. To increase the power, we also tested the pooled results of the three postures for each bony prominence using the ordinary least squares (OLS) test as described by $\mathrm{O}^{\prime}$ Brien $^{12}$ and modified by Läuter ${ }^{13}$.

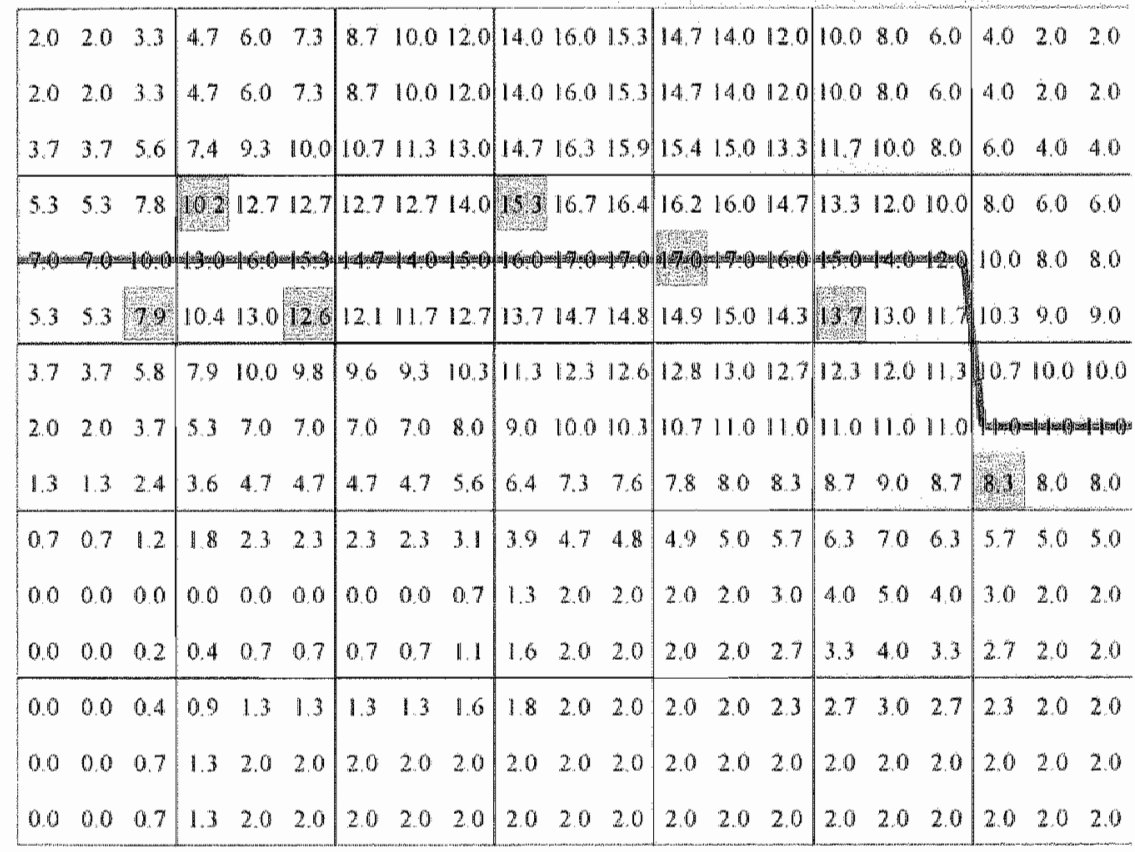

Fig. 3: The interpolated presstre matrix of the case presented in Figure 2. The light grey areas represent from left to right: the medial sesamoid, MTH 1 , lateral sesamoid, and MTH 2 to 5 . The "Ine of maximum prossure' (dark grey line) connects the areas with the maximum pressure in each wertical colum ([numbers] $=\mathbb{N} / \mathrm{cm}^{2}$ ). 


\section{Results}

The position of the bony prominences and the line of maximal pressure coincided progressively less often on consecutive postures: $51 \%(36 / 70), 31 \%(22 / 70)$ and $25 \%$ $(18 / 70)$ for respectively the loaded, heel-off, and toe-off posture. The corresponding frequency distribution of the distance (expressed as the number of areas) between the position of the bones and 'the line of maximum pressure' showed flattening on consecutive postures (Fig. 4).

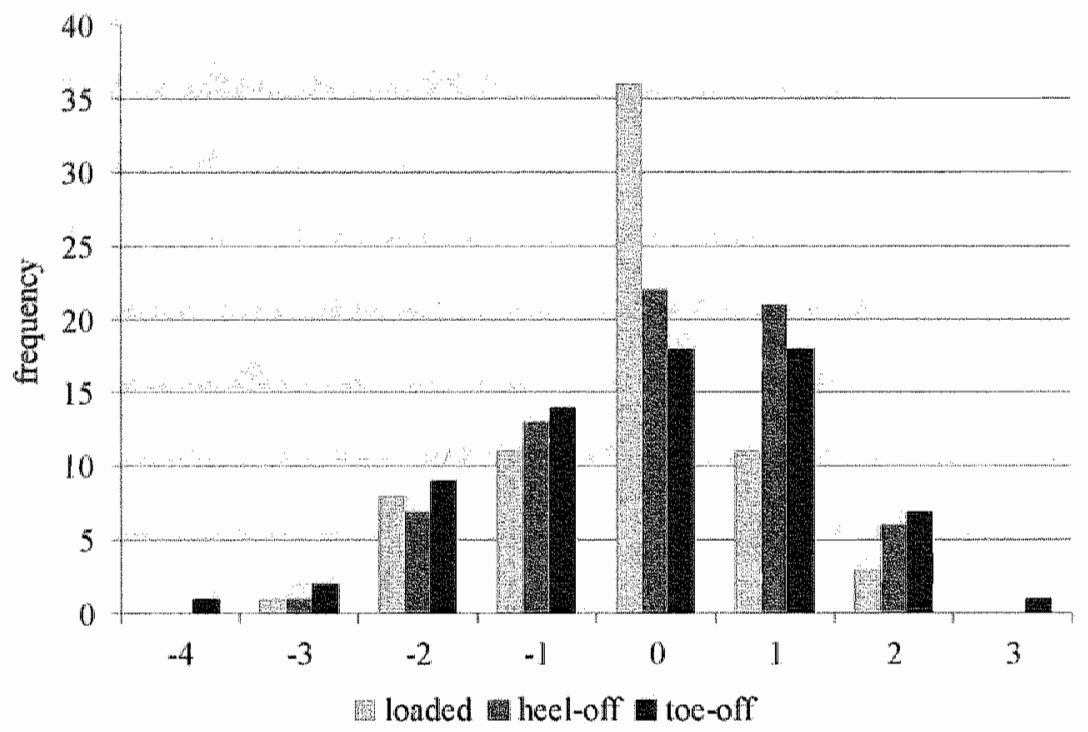

Fig. 4: The frequency distribution of the number of areas between the area of the bony prominence and the area of peak pressure. For each posture, the sewen bony prominences of all volunteers were incuded. 0: areas coincided; 1: areas adjoined; 2 ; one other area interposed; 3: two other areas interposed; 4: three other areas interposed. Negative numbers mean that the bone is positioned proximally to the line of maximat pressure.

The p-values of the one sample t-test and the OLS test on the pooled data were listed in Table 1. The p-values of the MTH 1,4 , and 5 as well as the MTH2 in the heel-off posture were less than 0.08 .

Table 1: P-values of the one sample t-lest an the distance between the bone and the line of maximum pressure and p-values of the 015 test on the pooled data. The p-values $<0.10$ are printed in bold. (MS: medial sesamoid: Ls: lateral sesanoid).

\begin{tabular}{llllllll} 
& MS & LS & MTH1 & MTH2 & MTH3 & MTH4 & MTH5 \\
\hline loaded & 0.68 & 0.18 & $\mathbf{0 . 0 5}$ & 0.34 & 0.56 & $\mathbf{0 . 0 5}$ & $\mathbf{0 . 0 2}$ \\
heel-off & 0.81 & 0.8 & $\mathbf{0 . 0 5}$ & $\mathbf{0 . 0 4}$ & 0.8 & $\mathbf{0 . 0 2}$ & $\mathbf{0 . 0 8}$ \\
toe-off & 1 & 0.5 & $\mathbf{0 . 0 1}$ & 0.31 & 0.8 & $\mathbf{0 . 0 8}$ & $\mathbf{0}$ \\
pooled & 0.83 & 0.49 & $\mathbf{0 . 0 4}$ & 0.23 & 0.72 & $\mathbf{0 . 0 5}$ & $\mathbf{0 . 0 3}$
\end{tabular}


The means of the difference in distance (in mm) of the positions of the MTHs, the sesamoids and 'the line of maximum pressure' for the three postures were illustrated in Figure 5. It showed that the MTH 1 was positioned $3 \mathrm{~mm}$ distally to "the ine of maximum pressure" and the MTH $54 \mathrm{~mm}$ proximally in the loaded posture. These distances increased to respectively 5 and $10 \mathrm{~mm}$ in the toe-off posture. Except for the sesamoids, the results suggested a correlation between the MTHs and distance. This was tested with the Spearman correlation coefficient for the three postures. The correlation coefficients increased with successive postures $(r=0.50,0.55,0.67)$ and showed to be significant $(p=0.002, p<0.001, p<0.001)$. The sesamoids were not included in the calculations of the Spearman correlation coefficient.

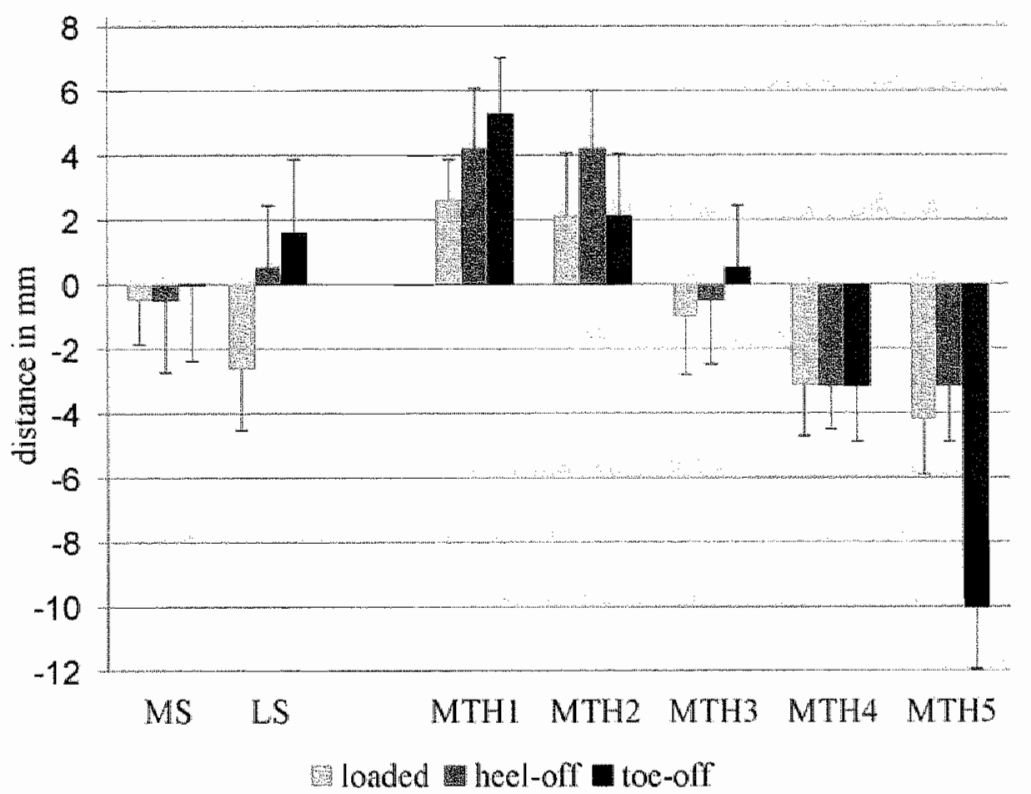

Fig. S: Results of the means (and their standard error) of difference in distance (in mm) between MTHs. (MTH

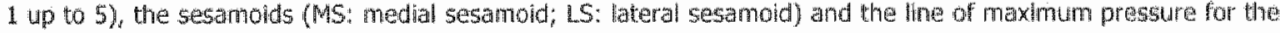
three postures.

\section{Discussion}

In this paper we tested the premise that the position of local peak pressures coincides with the position of the MTHs and sesamoids. Our results showed that the two only coincided in $51 \%, 31 \%$, and $25 \%$ of the cases for the consecutive postures (Fig. 4). Especially MTH 1, 4, and 5 as well as the MTH 2 in the heel-off posture showed a significantly different position than the corresponding local peak pressures (Table 1). Mioreover the average distances between the MTHs and 'the line of maximum pressure" showed a correlation that got steeper on consecutive postures (Fig. 5). The position of MTH 3 coincided with the corresponding local peak pressure and fitted into the correlation.

These observations suggest that the normal padding of the metatarsals is able to deflect load away from the most exposed parts of the metatarsals. At the level of the MTH the skin and subcutaneous tissue are anchored to the deeper layers of the foot by means of vertically 
oriented fibrous sheets. They originate from the tunnels around the flexor tendons and the joint capsule ${ }^{14}$. These structures not only protect the tendons and nerves of the toes by cushoning, but it is very likely they are also able to deflect the load away from the most exposed part of the MTHs. The efficiency of this deflection seems to improve with increasing extension of the toes. This might be reflected in the observation that in a relaxed state the soft tissue of the ball of the foot is pliable, but gradually becomes firmer on extension of the toes ${ }^{15.5}$. We found no explanation for the observation that the deflection of load is most pronounced on the medial and lateral foot border in respectively proximal and distal direction. Our observations relate to healthy volunteers. It is conceivable that degradation of the architecture of the ball of the foot (as in aging, atrophy, and trauma) or changes in the composition of its constituents (as in rheumatoid arthritis and diabetes) diminish the load deflective abilities of the plantar soft tissue ${ }^{16-18}$.

The sesamoids are embedded in the plantar plate of the great toe and a complex of flexor tendons. The plantar plate is considered as part of the padding soft tissue of the MTHs ${ }^{19}$. Therefore, the sesamoids might be considered as part of the soft tissue. Moreover the sesamoids show considerable interindividual variation in anatomy ${ }^{20}$ (bi- and even tripartite bones), number, and size ${ }^{21}$ and move relative to the metatarso-phalangeal joint on extension of the toes. These factors likely explain the different results of the sesamoids compared to those of the MTHs. They were not included in the calculation of the Spearman correlation coefficient.

The postures resemble three different phases of a walking cycle. However, they likely do not reflect the normal physiology of a walking cycle. Extrapolation of our results to a dynamic situation should be done with caution.

In all postures our method of measuring the position of the MTHs was sufficiently reproducible. The observed error is small in relation to the size of an interpolated area. The plantar pressures are measured with an accuracy of $5 \%$ over an area of approximately $1.8 \mathrm{~cm}^{2}$.

To be able to detect differences in positions of the local peak pressures and bony prominences one should pursue the highest spatial resolution of the measuring devices. Currently, pressure distribution measuring platforms supply the best spatial resolution. Those platforms contain metal, that would cause unacceptable artifacts on the CT images. Alternatively, we used an insole system that contained little metal at the cost of spatial resolution. The pressure recorded is an average of the pressure over that cell. It does not give information about the distribution of the true pressure over that cell. Depending on the size of the sensor as well as the steepness and height of the peaks of pressure, an underestimation up to $30-40 \%$ of the true peak can occur ${ }^{22}$. In normal wolunteers it is unusual to find a focal area of increased pressure ${ }^{23}$. Indeed, the plantar pressure profiles of our volunteers varied smoothlly. This justifies the use of interpolation to increase the spatial resolution.

Interpolation is a generally accepted concept in medical imaging processing and a variety of methods has been described ${ }^{24,25}$. We used a simple algorithm that should work properly for the smoothly varying plantar pressure profiles of healthy volunteers.

Therapeutic footwear, orthosis, and inlays attempt to relieve excessive pressure underneath the bony prominences ${ }^{3,5}$. The effectiveness of these depends on the position in relation to the MTHS ${ }^{7}$. The design is still based largely on experience and opinion rather than experimentally confirmed principles ${ }^{6 * 9}$. Our results might influence the design of therapeutic footwear, but our data relate to healthy persons, not patients.

In conclusion the MTHs have a different position than the local peak pressures measured at the surface of the sole especially on the medial and lateral side. This suggests that the normal padding of the MTHS is able to deflect a load away from the most exposed parts of the metatarsal heads and does so more efficient with increasing dorsiflexion of the toes. 


\section{References}

1. Cavanagh $P R$, Uibrecht IS and Caputo GM: New developments in the biomechanics of the diabetic foot. Diabetes Metab Res Rev 2000; 16 Suppil 1: S6-S10.

2. Ashry HR, Lavery LA, Murdoch DP, et al.: Effectiveness of diabetic insoles to reduce foot pressures. J Foot Ankle Surg 1997; 36: 268-271.

3. Chang $\mathrm{AH}$, Abu-Faraj $\mathrm{ZU}$, Harris $\mathrm{GF}$, et al.: Multistep measurement of plantar pressure alterations using imetatarsal pads. Foot Ankle Int 1994; 15:654-660.

4. Colagiuri S, Marsden LL, Naidu V, et al.: The use of orthotic devices to correct plantar callus in people with diabetes. Diabetes Res Clin Pract 1995; 28: 29-34.

5. Hayda $R$, Tremaine MD, Tremaine $K_{n}$ et al: Effect of metatarsal pads and their positioning: a quartitative assessment. Foot Ankle Int 1994; 15: 561-566.

6. Hodge MC, Bach TM and Canter GM: novel Award First Prize Paper. Orthotic management of plantar pressure and pain in rheumatold arthritis. Clin Biomech (Bristol, Avon) 1999; 14: 567-575.

7. Holmes GB, Jr. and Timmerman L: A quantitative assessment of the effect of metatarsal pards on plantiar pressures. Foot Ankle 1990; 11: 141-145.

8. Kelly A and Winson I: Use of ready-made insoles in the treatment of lesser metatarsalgia: a prospective randomized controlled trial. Foot Ankle Int 1998; 19:217-220.

9. Lemmon $D$, Shiang $T Y$, Hashmi $A_{n}$ et al: The effect of insoles in therapeutic footwear: a finite element approach. J Biomech 1997; 30; 615-620.

10. van Schie $C_{\text {, }}$ Ulbrecht $\mathrm{JS}_{4}$ Becker $\mathrm{MB}_{\text {n }}$ et al.: Design criteria for rigid rocker shoes. Foot Ankle Int 2000; 21 : 833-844.

11. Feibel $\mathrm{JB}$, Tisdel $\mathrm{CL}$ and Donley $\mathrm{BG}$ : Lesser metatarsal osteotomies. A biomechanical approach to metatarsalgia. Foot Ankle Clin 2001; 6: 473-489.

12. O'Brien PC: Procedures for comparing samples with multiple endpoints. Biometrics 1984: 40: 1079-1087.

13. Lauter ]: Exact $t$ and F-test for analyzing studies with multiple endpoints. Biometrics 1996; 52: 964-970.

14. Bojsen-Moller $F$ and Flagstad KE: Plantar aponeurosis and internal architedure of the ball of the foot. J Anat 1976; 121: 599-611.

15. Bojsen-Moller F and Lamoreux L: Significance of free-dorsiflexion of the toes in walking. Acta Orthop Scand 1979; 50: 471-479.

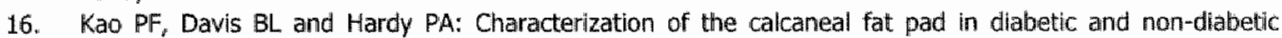
patients using magnetic resonance imaging. Magn Reson Imaging 1999; 17: 851-857.

17. Buschmann WR, Jahiss $M H_{r}$ Kummer $F_{\text {, }}$ et al.: Histology and histomorphometric analysis of the nomal and atrophic heel fat pad. Foot Ankle Int 1995; 16: 254-258.

18. Rome K: Mechanical properties of the heel pad: current theory and review of the literature. The Foot 1998; 8: $179-185$,

19. Deland $\mathrm{JT}$, Lee $K T$, Sobel $M_{\text {, et }}$ al: Anatomy of the plantar plate and its attachments in the lesser metatarsal phalangeal joint. Foot Ankle Int 1995; 16: 480-486.

20. Prieskorn $D_{y}$ Graves SC and Smith RA: Morphometric analysis of the plantar plate apparatus of the first metatarsophalangeal joint. Foot Ankle 1993; 14: 204-207.

21. Saraffian SK: Anatomy of the Foot and Ankle,second; Lippincot 1.B., Philadelphia 1993.

22. Lord M: Spatial resolution in plantar pressure measurement. Med Eng Phys 1997; 19: 140-144.

23. Cavanagh $P R$, Rodgers MM and Iibashi $A$ : Pressure distribution under symptom-free feet during barefoot standing. Foot Ankle 1987; 7: 262-276.

24. Thevenaz P, Blu T and Unser M: Interpolation revisited. IEEE Trans Med Imaging 2000; 19: 739-758.

25. Lehmann TM, Gonner $\mathrm{C}$ and Spitzer $\mathrm{K}$ : Survey: interpolation methods in inedical image processing. IEEE Trans Med Imaging 1999; 18: 1049-1075. 


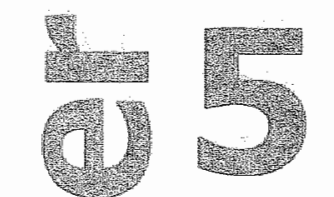

\section{Plantar pressure and sole thickness of the forefoot}

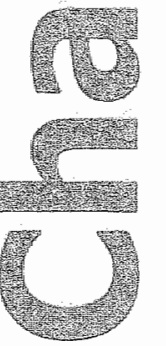

RE Weijers, GHIM Walenkamp, AGH Kessels, GJ Kemerink, and $H$ van Mameren

Foot and Ankle International, accepted for publication

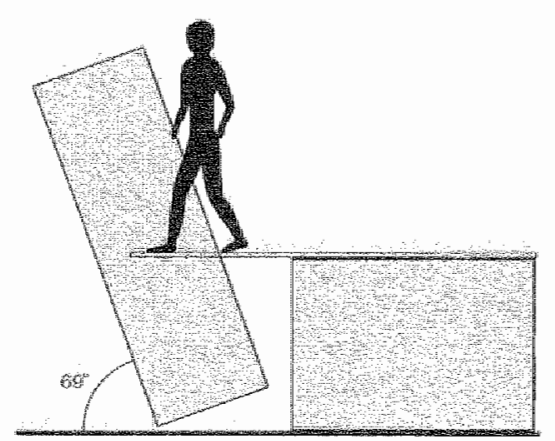




\section{Abstract}

Background: Based on earlier observations on the forefoot that its centre bears the highest plantar pressure, the existence of a functional distal transverse arch in normal feet was denied. But, plantar pressure is merely the outcome of loading, surface area, and soft tissue. Although it drives the changes in the foot; neither the position nor the behaviour of the metatarsals during loading can be derived from plantar pressure alone. In light of this, our goal was to describe the changes in thickness in the sole and the corresponding plantar pressure during loading of the foot.

Methods: We used a CT scanner to image the foot of ten subjects in four postures that were chosen to imitate four phases in the walking cycle. Prior to imaging we also recorded the plantar pressure with a pressure measuring insole on which the subjects were standing. From the data the minimal thickness of the sole and the corresponding plantar pressure could be derived.

Results: With the exception of the sesamoids, the thickness of the sole under the bones of the forefoot increased from lateral to medial. This persisted in all postures. Our pressure readings matched previously reported distributions.

Conclusions: Depending on the point of view concerning the sesamoids, the bony prominences were placed in geometrical arch; just the same they did not form a functional arch. The soft tissue underneath the heads kept them in place, its thickness reflected the principle of adequate cushioning. 


\section{Introduction}

To avoid structural damage to the foot, the impact forces related to locomotion are dampened and energy is absorbed. To aid this process both the bones that are arranged in several arches and the soft tissue of the sole of the forefoot contribute. In this paper we will discern a geometricall and a functional arch. The geometrical arch merely describes the curved arrangement of an array of bones. The top of this curve is located somewhere in the middle of the arch, not at its far ends. A functional arch has the same features as the geometrical arch, but it also disperses a load. As a consequence the constituting bones of such a functional arch should be interconnected in such a way that the load can be transferred in the direction of the pillars that are positioned at the far ends of the arch. The pillars should be stably positioned in vertical and horizontal position.

Traditionally, in the foot skeleton three functional arches were distinguished: a medial longitudinal one involving the calcaneus, talus, navicular, cuneiforms and the three medial metatarsals, a lateral longitudinal one involving the calcaneus, cuboid and the two lateral rays, and finally a transverse arch involving the metatarsal heads. The latter is not to be confused with the contemporary definition of the transverse arch which is located more proximally and involves the base of the metatarsal bones, the cuneiforms and the cuboid ${ }^{1-3}$. In the past the concept of a functional distal transwerse arch through the metatarsal heads was widely accepted. It was thought that this structure played a major structure in the absorption of energy ${ }^{4}$.

With the advent of devices to measure plantar pressure, the peak plantar pressure in the normal forefoot was determined to be highest under the second or third metatarsal head both during stance and locomotion ${ }^{547}$. These findings were not in accord with the concept of a functional arch through the metatarsal heads because in that case the highest peak plantar pressures should be located under the first and fifth metatarsal head. Based on this reasoning the existence of a functional transverse arch was negated. From this conclusion an explanation for the anatomical arch-like form of the forefoot cannot be derived. Moreover, pressure readings do not give information about the thickness or the structure of the soft tissue of the sole. Therefore the goal of our study was to analyse the apparent contradiction of form and function of the forefoot by describing the thickness of the sole and the changes thereof during loading in relation to plantar pressure, a matter on which only limited information is available ${ }^{8,9}$. For this purpose we simultaneously used a CT-scanner and a pressure measuring insole.

\section{Materials and methods}

Ten subjects were selected: five males and five females. In each decade of age, from the second to the fifth, a male and a female were represented (mean 34 years, range 19-51). They were healthy subjects, without complaints of their feet, callus, deformation, or a history of previous trauma or surgery. Height, weight, nor foot size were inclusion/ exclusion criteria. The study was approved by our Institutional Review Board. All subjects gave informed consent.

A pressure measuring insole was mounted on a perspex plank. In this plank holes of a diameter of $1 \mathrm{~mm}$ were vertically drilled forming an orthogonal coordinate system. The plank was fixed to the tabletop of a dual slice CT scanner with the gantry maximally tilted $\left(21.2^{\circ}\right)$. The pressure measuring device was calibrated immediately prior to each experiment.

During the experiment the subjects were standing upright on bare feet, with the right foot in front of left, on the tabletop of the spiral CT scanner. The right foot was positioned on the insole with the axis of the second metatarsal bone parallel to the side border of the tabletop ${ }^{10,11}$. 
For each subject, four consecutive scans of the right forefoot were made in four different postures that were chosen to imitate four phases in a walking cycle. Between the scans the sole of the foot did not lose contact with the tabletop. During the fifteen seconds immediately prior to the start of each scan we recorded the pressure distribution. The subjects were instructed to freeze their posture during pressure reading and imaging.

In the first posture the right foot of the subjects just touched the surface of the insole, while the left foot supported the body weight: the unloaded posture. Next, the subjects shifted the body weight to the right foot as much as possible: the mid stance posture. The left foot just touched the surface of the tabletop to help the subject to keep their balance. The scan was started when the maxirnum pressure in the region of the ball equalled the maximum pressure under the heel reglion for a period of fifteen seconds. Then the subjects loaded the forefoot as much as possible while the heel just remained in contact with the insole: the heel-rise posture. The third scan was started when the recorded maximum pressure over the forefoot was constant and the pressure over the heel was about $2 \mathrm{~N} / \mathrm{cm}^{2}$ at most for a period of fifteen seconds. During the last scan the subjects gave maximum pressure on the forefoot while extending the foot with as much heel-rise as possible: the toe-off posture. To give the patient visual feed back on the plantar pressure, the subjects could see the monitor of the Pedar system while standing on the tabletop.

The spiral scan (Elscint, Old Delft, Defft, the Netherlands) of the forefoot included all the metatarso-phalangeal joints and ranged from the first tarso-metatarsal joint till the most distall interdigital web. For technical details we refer to previous publications ${ }^{16,17}$.

The insole (Pedar system, Novel electronics inc., St Paull, MN, USA), European shoe size 46, measured the normal interface stress $\left[\mathrm{N} / \mathrm{cm}^{2}\right]$ with ninety-nine sensors. The average size of the sensors over the forefoot region was $1.8 \mathrm{~cm}^{2}$. The accuracy of measurement was better than $5 \%$ (as specified by the manufacturer).

We defined the thickness of the sole as the minimal vertical distance between the bony cortex of the metatarsal head or sesamoid and the underlying surface. The thickness was measured on a 3D multi-planar reformat workstation (EasyScil 4.2.1, Philips, Best, The Netherlands): the point in space where the cortex protruded most, was marked in the scanned volume and the vertical distance to the underlying surface was semi-automatically measured. This procedure was carried out twice for each metatarsal head and sesamoid to estimate the reproducibility of this method. The median of the difference of the first and second measurement of thickness of the sole was $-0.01 \mathrm{~mm}$ (5 percentile: $-.55 \mathrm{~mm}$; 95 percentile: $40 \mathrm{~mm}$ ). The average of both measurements was finally used for the thickness. From the thickness we derived:

- the indentation: the difference in thickness of the sole in the unloaded and the loaded postures (indentation $=$ thickness unloated - thickness loaded)

- the strain: the indentation expressed as a percentage of the unloaded thickness ([thickness unloaded - thickness loaded] $100 /$ thickness unloaded).

To link the data of the pressure distribution to the thickness of the sole, the position of both the comers of the pressure measuring elements of the insole and the point of minimal soft tissue thickness were expressed as coordinates in the orthogonal coordinate system of the perspex plank. The coordinates of the corners of the pressure sensitive elements of the insole were directly measured on the orthogonal coordinate system in the plank. On the workstation the (already) marked points of minimal soft tissue thickness were vertically projected on the (horizontal) plane of the plank and the coordinates were read out on the orthogonal coordinate system. For further details on this method, we refer to a previous

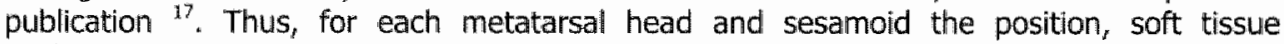
thickness, strain, and corresponding pressure (stress) were determined. These data were used to construct the curves of a stress-indentation graph. This graph was used to indicate 
the energy absorption and soft tissue stiffness under each metatarsal head. The energy absorption correlates with the area under the curve (work equals force times distance), the stiffness with the steepness of the curve.

Whether the soft tissue thickness increased from lateral to medial was tested for each posture with univariate linear regression analysis. The differences in thickness, strain, and stress between the postures were tested with multivariate regression analysis with 'posture' as a dummy variable. Because of the form of the curves we used a linear regression model for thickness and a quadratic one for strain and stress.

\section{Results}

The mean thickness of the sole under the metatarsal heads was progressively lower from medial to lateral for all four postures (regression coefficient: $-1.4 ;-1,0 ;-9 ;-.7$ for respectively the unloaded, mid stance, heel-rise and toe-off postures; all p's < .0005) (Fig. 1). The tissue under the sesamoids was thinner than under MT 1. Considering the consecutive postures, the sole thinned statistically significantly between the unloaded and the mid stance posture $(p<.0005)$. Between the other postures the effect was smaller and not statistically significant.

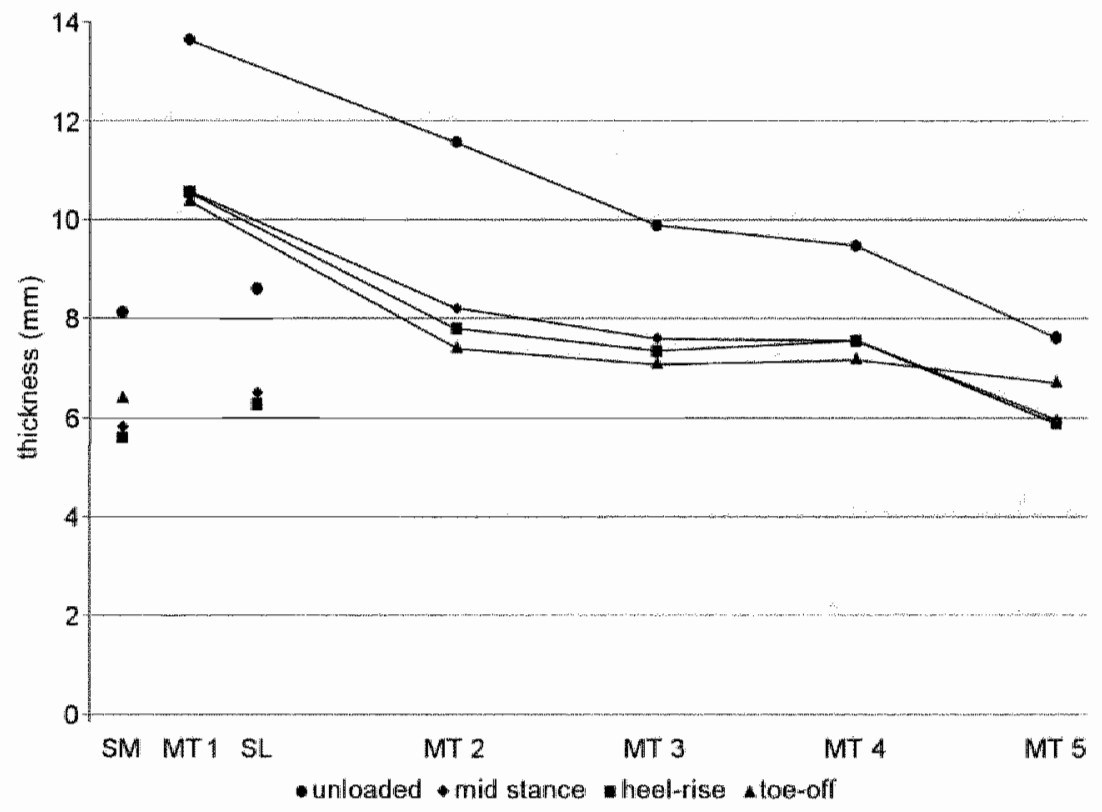

Fig. 1: Thickness (mm) of the sole under the bony prominences of the forefoot in all fout postures. (5M: madiat sesamoid: SL: lateral sesamoid; MT 1-5; metatarsal head 1-5). 


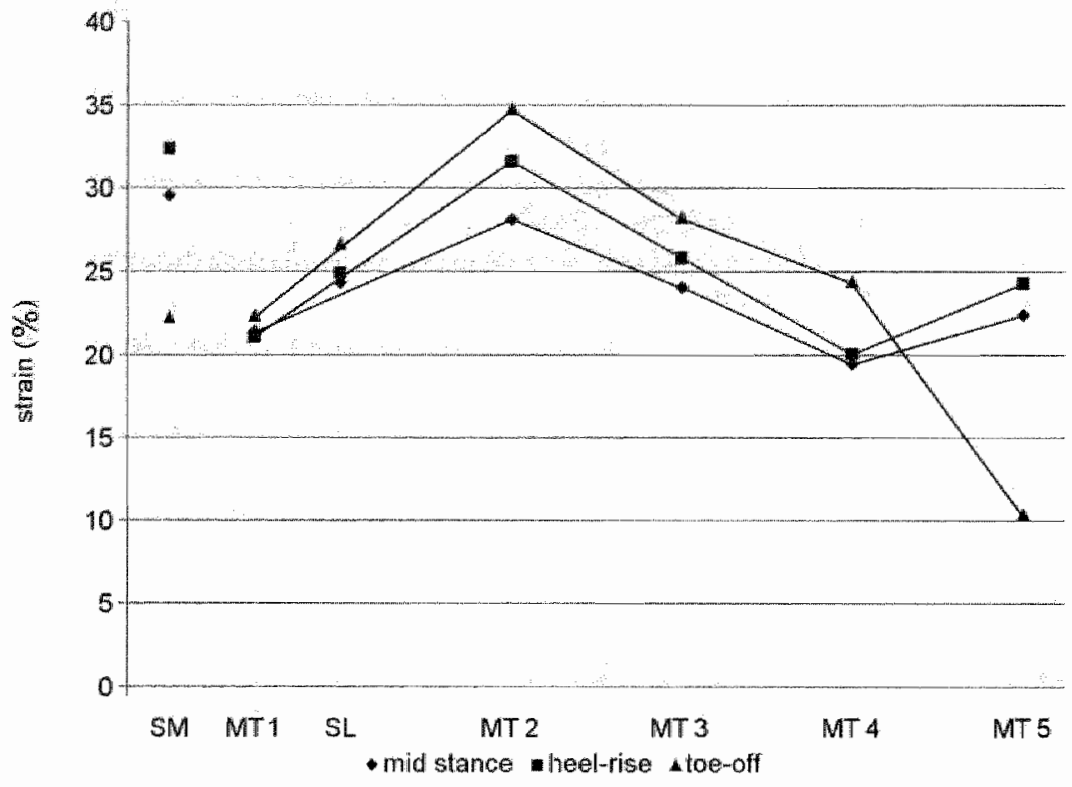

Fig. 2. Strain (indentation expressed as percentage of the unloaded soft tissue thickness (100* mom/mm]) of the soft tissue of the sole under the bony prominences of the forefoot in the three weight bearing postures. (SMi: medal sesamaid; SL: lateral sasamoid; MT 1*5: metatarsal head $1-5$ ).

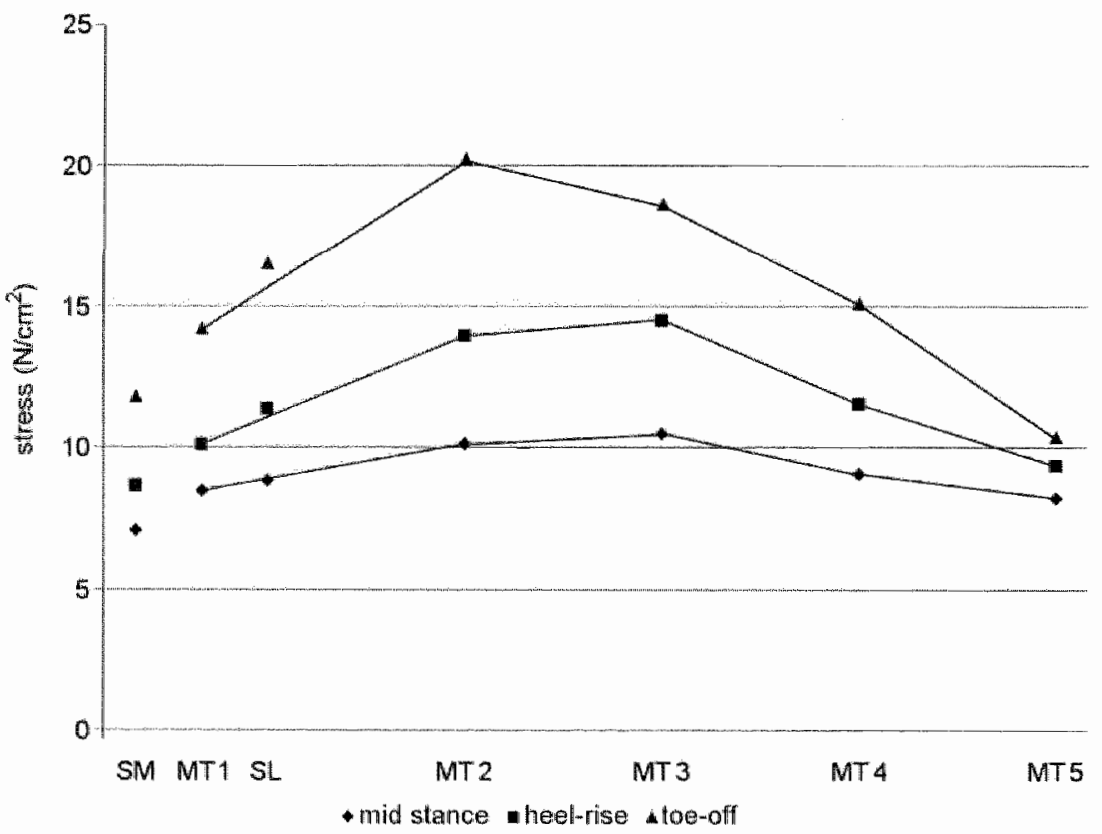

Fig. 3: Stress (N/ $\left./ \mathrm{cm}^{2}\right)$ under the sole at the bony prominences of the forefoot in the three weight bearing postures. (SM: medial sesanoid; SL: dateral sesamoid; MT 145: metatarsal head 1-5). 


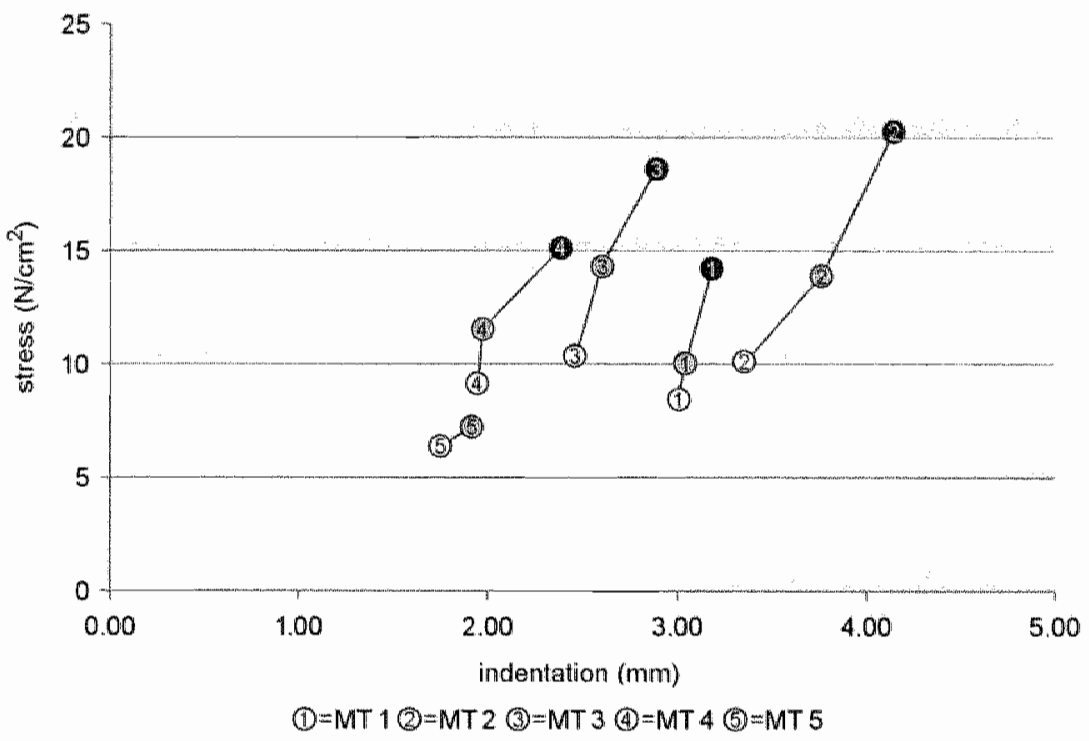

Fig. 4: Stress [N/ $\left.\mathrm{cm}^{2}\right]$ versus indentation [mm] diagram of the soft tissue of the sole under the bony prominences of the forefoot (MT 1-5: metatarsal head 1-5). The area under the cunes is a measture for the absobed energy (work equals force limes distance). The steepness of the curve is a macure of the stiffress. The open symbols refer to the mid stance posture, the grey symbols to the healrise posture, and the black symbols to the toe-aff posture. The data of the toemofl of MT 5 are not included (see discussion).

The mean strain under the metatarsal heads increased in successive phases (Fig. 2). It was most pronounced under MT 2 in all postures. However, the results were not significant $(p>.1)$. In the toe-aff posture neither MT 5 nor the medial sesamoid followed the trend of the other metatarsals. MT 5 was left out of the statistical analysis of strain.

The stress (= plantar pressure) under the bony prominences of the forefoot is Illustrated in Figure 3 . The stress increased in successive postures progressively (mid stance - heel-rise: $p=.05 ;$ heel-rise - toe-off: $p=.003$ ). The highest stress during mid stance and heel-rise posture was measured under MT 3. In toe-off posture it shifted medially to MT 2. The stressindentation diagram suggests that during loading most energy is absorbed by MT 2 (Fig. 4).

\section{Discussion}

$C T$ in combination with a pressure measuring device offered a unique opportunity to acquire data on both the thickness of the sole of the forefoot and the corresponding pressure.. The CT-methad to measure the thickness of the sole proved to be reproducible. Despite the four different postures, mimicking phases in a walking cycle, the nature of the study remained static. As a consequence, the acquired data cannot be fully extrapolated to a normal dynamic stride.

In the past the concept of a functional transverse arch through the five metatarsal heads was generally accepted. This arch was supposed to play a role in energy absorption and stress reduction ${ }^{4}$. When this supposed transverse arch would be functional, it would require the pressure under the first and fitth metatarsal heads to be higher than under the mid- 
forefoot. Several authors ${ }^{5.7}$ showed that MT 2 and 3 bear the highest plantar peak pressure both during standing and walking. Our data confirm these findings (Fig. 3). Based on their results of pressure measurement only, Cavanagh ${ }^{7}$ and Luger ${ }^{*}$ concluded that there is no functional anterior transverse arch. Even more, it was suggested that: "A transwerse arch indicates a possible pathological deformity' ${ }^{6}$. This reasoning led to the current opinion that in normal feet neither a functional nor a geometrical transwerse arch exists during weight bearing. But, as aiso put forward by Harris ${ }^{12}$, plantar pressure is merely a reactive force. It dependis on the loading on the foot that is transmitted and altered by the internal supporting structureis of the foot; the thickness and internal structure and the surface area of the soft tissue of the sole. Obviously, there will not be a fixed relationship between plantar pressure and the position of the metatarsal heads, or the thickness of the sole. Therefore, data on plantar pressure alone cannot exclude the existence of a geometrical transverse arch. However, the position of the metatarsal heads is accurately indicated by the soft tissue underneath. To make out whether a geometrical transverse arch exists, we described the soft tissue of the sole and its reaction to load.

Like Hermann ${ }^{8}$ and Wang ${ }^{9}$ who used ultrasound as a measuring modality, we observed that the sole under the lesser metatarsal heads thickened progressively from lateral to medial in both the unloaded and standing/loaded posture (Table 1). Moreover, we found that this distribution was maintained in heel-rise and toe-off posture (Fig. 1). Only at MT 5 in the toeoff posture the mean thickness increased in five out of ten subjects. These subjects extended the foot so much that the head of MT 5 rose while the plantar skin remained in contact with the tabletop. Under MT 1 , Hermann "reported a smaller thickness than under MT 2 and Wang ${ }^{9}$ the opposite. This is explained by a methodological difference: Hermann ${ }^{8}$ used the sesamoids and Wang ${ }^{9}$ the first metatarsal head as the bony reference to measure the thickness of the sole. We confirmed the findings of MT 1 of both authors using CT (Table 1). In general we measured the sole about $1 \mathrm{~mm}$ thicker than Hermann ${ }^{8}$ and about $2 \mathrm{~mm}$ thinner than Wang in both the unloaded and the standing/loaded position (Table 1).

Table 1: Summary of the soft tissue thickness (in $\mathrm{mm}$ ) under de metatarsil heads (MT1-5) and sesamoids (SM: medial sesamoid; 5 : lateral sesamoid). The data of Hermann ${ }^{3}$ are derlvad from his graphs, the data of Wang from the average values of his tables. The loaded data of Weijers refer to the mil stance posture.

\begin{tabular}{llccccccc}
\hline \multirow{2}{*}{ Hermann } & & SM & SL & MT1 & MT2 & MT3 & MT4 & MT5 \\
\cline { 5 - 9 } & unloaded & & 5.9 & & 10.3 & 9.2 & 7.5 & 7.2 \\
\cline { 2 - 9 } Wang & loaded & & 5 & & 8 & 6.8 & 6.3 & 5.2 \\
\hline \multirow{2}{*}{ Weijers } & unloaded & & & 15 & 13.6 & 12.5 & 11.4 & 10.4 \\
\cline { 2 - 9 } & loaded & & & 8.4 & 7.2 & 6.4 & 6 & 5.4 \\
\hline
\end{tabular}

The difference with Hermann ${ }^{8}$ is likely due to our younger population of subjects. There is no obvious explanation for the difference with Wang, except perhaps racial. On all consecutive weight bearing postures the sole under the metatarsal heads progressively thinned, but most pronounced between the unloaded and mid stance posture (Fig. 1 and 2). This is in accordance with observations on the heel pad that most thinning occurs in the early phases of loading ${ }^{13 ;}, 14$.

The methodological difference as mentioned above is relevant to the question whether the metatarsals form a geometrical arch. The sesamoids are embedded in the plantar plate at MT 1 a fibrocartilagenous structure that is also present under the lesser metatarsal heads ${ }^{15,16}$. 
The mechanical characteristics of the plantar plate differ from the rest of the plantar soft tissue ${ }^{15}$. If the soft tissue under MT 1 was measured the same way as the soft tissue under" the lesser metatarsals ${ }_{r}$ as Wang ${ }^{9}$ did, the sesamoids were considered to be part of the soft tissue. In that case metatarsal heads do not form a geometrical arch. If the soft tissue thickness under the most protruding bones of each ray is measured, the sesamoids are part of the bony structure of the foot. In that case bones of the forefoot form a geometrical arch involving the medial sesamoid, the lateral sesamoid, and the heads of MT 2-5, as concluded

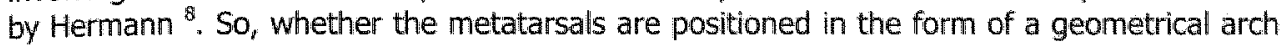
is a matter of opinion (Fig. 1).

The mean strain varied between $19-35 \%$ and decreased in the following order: MT 2 $>3>5>1>4$ (Fig. 2). This order is maintained in all postures (except MT 5 in toe-off posture). Both the magnitude and the order of the average strain were consistent with the data of Hermann ${ }^{8}$, but inconsistent with Wang's ${ }^{9}$ (Table 2). We calculated approximately half the values of his data. The difference in design of the experiments explained this. The formal definition of strain assumes a constant cross-section of the column of tissue of which the strain is measured. Our data and those of Hermann ${ }^{8}$ were measured while standing on the entire surface of the (fore)foot with loadings up to maximally $20 \mathrm{~N} / \mathrm{cm}^{2}$. Wang ${ }^{9}$ used an ultrasound transducer with a contact area of only $5.02 \mathrm{~cm}^{2}$, to apply pressures up to only $5.98 \mathrm{~N} / \mathrm{cm}^{2}$ to the plantar soft tissue thickness over the metatarsal heads. A smaller contact area allows compressed tissue to diverge sideways more than a larger area, giving thinner sole at lower loading. The data of Hermann and our data therefore reflect normal stance and walking better than those of Wang. However, the difference is not without meaning: While stepping on a pebble, the soft tissue behaves much more pliable than walking on a flat surface, avoiding (extreme) peak pressure and possible injury.

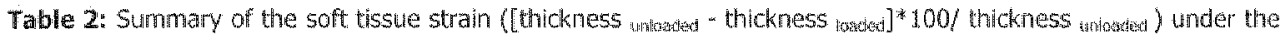
metatarsal heads (MTL-5) and sesamolds (SM: medial sesamoid; SL: lateral sesamoid) on maximal loading. The data of Wang ${ }^{9}$ were measured at a maximal loading of $5.98 \mathrm{~N} / \mathrm{cm}^{2}$ on a small areal. The data of Hermann ${ }^{\text {gt }}$ and our data refer to the mid stance posture of which the maximal load varted up to $20 \mathrm{~N} / \mathrm{cm}^{2}$.

\begin{tabular}{|c|c|c|c|c|c|c|c|}
\hline & SM & $S L$ & MT1 & MT2 & MT3 & MT4 & MT5 \\
\hline Hermann & & 15 & & 22 & 26 & 16 & 28 \\
\hline Wang & & & 44.1 & 47.3 & 49.2 & 47.5 & 48.5 \\
\hline Weijers & 28.2 & 23.7 & 21.3 & 28 & 22.6 & 19.1 & 21.8 \\
\hline
\end{tabular}

As is common practice in analysing mechanics of materials, another informative way of presenting the available data is a stress-indentation diagram. It gives an idea of both the stiffness (steepness of the curve) and energy absorption (area under the curve) of the stressed material. Of the heel pad, the stiffness was reported to be non-linear $1.3,17$; during the early phase only a small strain thins the sole considerably. Progressively more strain is required to thin the sole further, giving a steep upslope. Wang ${ }^{9}$ noted the padding of the forefoot to behave similar to the heel pad. He showed only one example of a stressindentation curve. At about $4-5 \mathrm{~mm}$ indentation, the flat part changed into the steep part of the curve. We had anly a limited number of measuring points, but our data suggested the transition to take place at a smaller indentation (Fig. 4). This difference would mean a stiffer sole of the forefoot when loaded fully instead of locally. The explanation of this observation is based on the same principles as the explanation of the observed difference in the results of strain. 
Figure 4 also suggested that the energy absorption during loading was most pronounced at MT 2, followed by MT $1,3,4$, and 5. To our knowledge this was not reported previously. This observation illustrated that most padding was present at the regions of the highest loading. Moreover, it corresponds with the predilection site of metatarsal stress fractures. These data should be interpreted with caution because our experiment related to a static situation and does not take the rate of loading into account.

In summary we concluded that in the forefoot it is disputable whether the metatarsals form a geometrical arch. Plantar pressure readings proved that the metatarsals do not form a functional arch. They are kept in position by supporting soft tissue of the sole of the forefoot. The differences in the thickness of the soft tissue of the sole of the foot express the biological need of adequate padding of pressure points.

\section{References}

1. Palastange $N$, Fleid $D$ and Soames $R$ : Structure and function.second; Butterworth-Heinemann, Oxford 1997.

2. MCMinn R, Gaddum-Rosse P, Hutchings R, et al.: MCMinn's Functional and Clinical Anatomy, Mosby, London 1995.

3. Moore $\mathrm{K}$ and Agur A: Essential Clinical Anatomy, Williams and Wilkins, Baltimore 1996.

4. Kapandji IA: The physiology of joints.E S Livingstone, Edinburg \& London 1970.

5. Hennig EM and Rosenbaum D: Pressure distribution patterns under the feet of children in comparison with adults. Foot Ankle 1991; 11: 306 311.

6. Luger EJ, Nissan M, Karpf $A$, et al.: Patterns of weight distribution under the metatarsal heads. Ji Bone Joint Surg Br 1999; 81: 199-202.

7. Cavanagh $P R$, Rodgers $M M$ and liboshi A: Pressure distribution under symptom-free feet during barefoot standing. Foot Ankle 1987; 7:262-276.

8. Hermann B: Form und Statik der" Metatarsalkopfchenreihe beim Erwachsenen. Sonographische und podometrische Untersuchung. Z Orthop Ihre Grenzgeb 1995; 133: 335-340.

9. Wang $\mathrm{CL}$, HSu TC, Shau YW, et al.: Ultrasonographic measurement of the mechanicall properties of the sole under the metatarsall heads. J Orthop Res 1999; 17: 709-713.

10. Weijers $\mathrm{RE}_{s}$, Walenkamp $\mathrm{GH}_{*}$ van Mameren $\mathrm{H}_{\text {, }}$ et al.: The relationstip of the position of the metatarsal heads and peak plantar pressure. Foot Ankle Int 2003; 24: 349-353:.

11. Weijers RE, van Mameren $\mathrm{H}_{s}$ Walenkamp $\mathrm{GH}_{s}$ et al.: Changes of the soft tissue of the forefoot during loading: volumetric study. Foot 2003; 13:70-75,

12. Harris $\mathbb{N J}_{3}$ Chell $\mathrm{J}$ and Smith TW: Patterns of weight distribution under the metatarsal heads. J Bone Joknt Surg Br 1999; $81: 744$.

13. De Clereq $D$, Aerts $P$ and Kunnen M: The mechanical characteristics of the human heel pad during foot: strike in running: an in vivo cineradiographic studly. J Biomech 1994; 27: 1213-1222.

14. Hsu TC, Wang $\mathrm{CL}_{\text {, Tsai }}$ WC, et al: Comparison of the mechanical properties of the heel pad between young and alderly adults. Arch Phys Med Rehabil 1998; 79: 1101-1104.

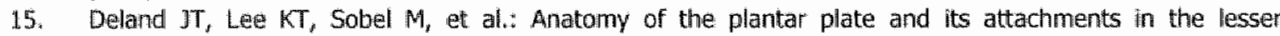
metaltarsal phatangeal joint. Foot Ankle int 1995; 16: 480-486.

16. Johnston RB, 3rd, Smith $\mathrm{J}$ and Damiels $\mathrm{T}$ : The plantar plate of the lesser toes: an anatomical study in human cadavers. Foot Ankle Int 1994; 15; 276-282.

17. Aents $P, K e r$ RF, De Clencq $D$, et al.: The mechanical properties of the human heel pad: a paradox resolved. J Blomech 1995; 28: 1299-1308. 


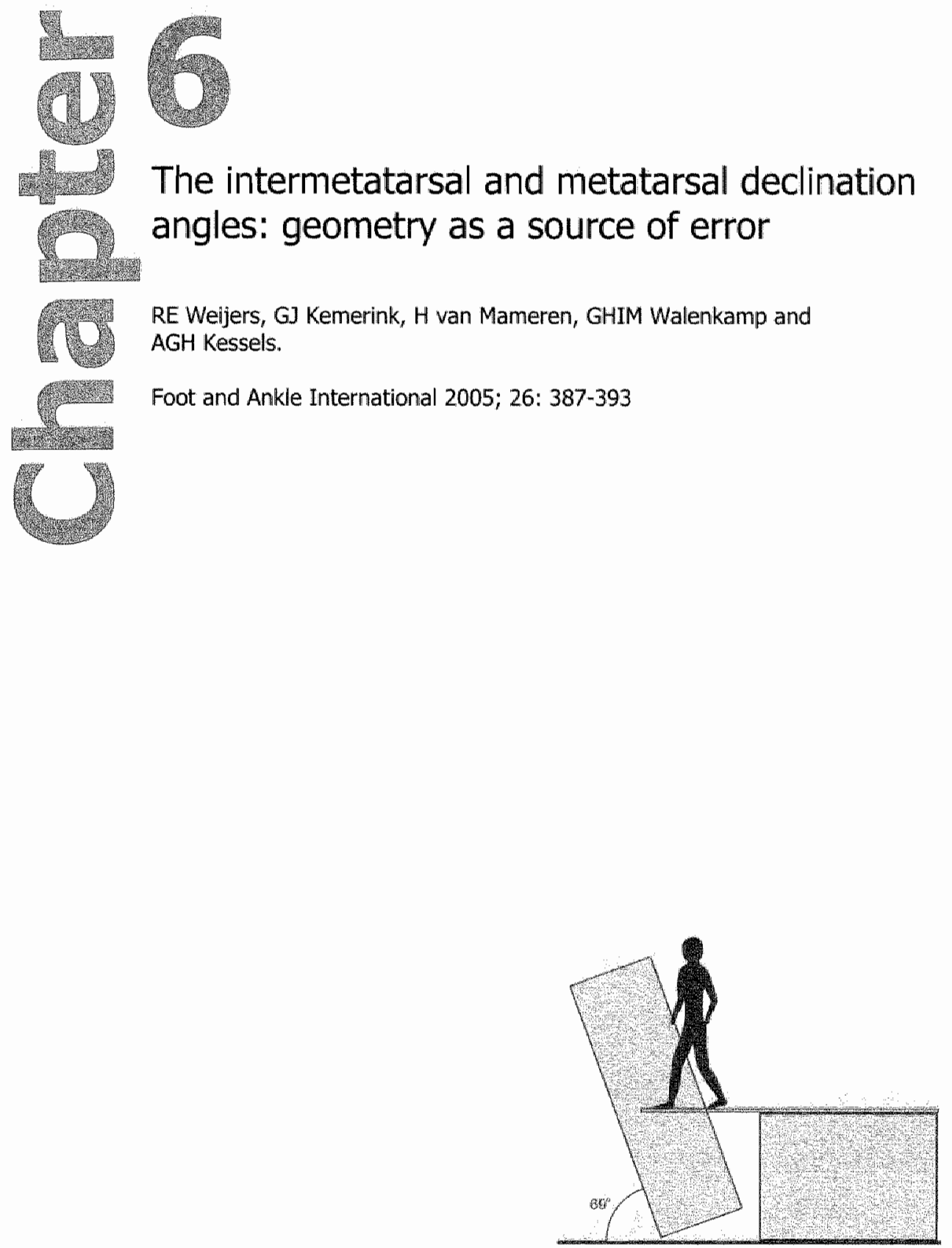




\section{Abstract}

Background: The measurements on radiographs of the foot are believed to give an objective impression of foot deformities. In patient management, the outcome of these measurements is one of the factors that the physician takes into consideration In fact, the radiograph is a projection of three-dimensional

such liable to a certain amount of distortion. To our knowledge this distortion has not been quantified, although it seems important for the interpretation of the measurements.

Methods. First, mathematical models based on the geometry of a standard anteroposterlor and a lateral radiographs were constructed and analysed. Then, we simulated radiography with these models on three-dimensional data of metatarsals originating from CT scans of 10 normal volunteers.

Results: The distortion of the declination angles of the metatarsals was practically negligible. The intermetatarsal angles were distorted up to $5.4^{\circ}$. The measured angles underestimated the threedimensional angles.

Conclusions, In interpreting the measurements of the metatarsal declination angles, distortion was not a factor. For intermetatarsal angles variability of distortion was only about one third of the reported interobserver variability, but it may have cumulative effects on the error of measured intermetatarsal angles, Moreover, the theoretical models predicted larger distortions in patients with deformities. In interpretation of angular measurements the physician should be aware of these pitfalls. 


\section{Introduction}

In patients with deformities of the forefoot, measurements on radiographs of the foot are used to indicate the severity of the deformation. These measurements are supposed to accurately reflect the foot's true three-dimensional (3D) bony structure, and they are considered in the choice of treatment ${ }^{1}$, in the evaluation of the results of treatment ${ }^{2}$, and in follow-up of a deformity. Because of this clinical importance attached to the measurements; earlier articles described various sources of error associated with different methods of measurement ${ }^{2-10}$ "Most attention was paid to the intra- and inter-individual variability of linear and angular measurements on radiographs ${ }^{3-5,8,11}$. But a radiograph is the result of a projection of 3D bones, and therefore the lengths and angles on radiographs are subject to distortion. The distortion related to linear measurements has been quantitatively analysed ${ }^{1 ?}$ " but to our knowledge, the distortion related to angular measurements has not been studied. Nevertheless, this is relevant because surgical treatment aims at restoring the spatial relation of the structures involved.

To determine how angular measurements of the metatarsals might be affected by distortion, we simulated lateral and anteroposterior (AP) radiographs of the foot. First, we formulated a theoretical assessment of how the variables describing the metatarsal geometry translate into distortion. Next, we analysed the distortion in dailly practice by simulating radiography on the data of computed tomography (CT) scans of the forefoot of normal volunteers.

\section{Material and methods}

The size of metatarsal angles as measured on a radiograph can be affected by error in measurement technique and by the orientation of the bones in 3D space in relation to the position of the roentgen tube and the film. To illustrate the contribution of distortion to the outcome of radiographic measurement, we analysed how distortion might vary with the orientations of the separate metatarsals, given the geometry of the standard radiographs (theoretical assessment). In reality, however, these variables simultaneously affect the distortion, so we simulated radiography on CT data of normal volunteers to calculate the amount of distortion in daily practice (clinical assessment).

\section{Definitions}

In this study the following definitions and abbreviations are:

- Lateral radiograph: radiograph of the foot of a standing individual, using a horizontal, mediolateral directed beam, centred at the lateral cuneiform bone and a focus-film distance of $1 \mathrm{~m} .{ }^{13}$ The film is vertically placed, parallel to the second metatarsal.

- Anteroposterior (AP) or dorsoplantar radiograph: radiograph of the foot of a standing individual, using a $15^{\circ}$ craniocaudal angled beam, centred at the second tarsometatarsal joint and a focus-film distance of $1 \mathrm{~m} .{ }^{14}$

- Declination angle (DA): the angle between the shaft of the metatarsal and the supporting plane. We distinguished two kinds:

- the measured declination angle (measured-DA): the simulated declination angle as measured on a lateral radiograph of the foot.

- the 30 decination angle (3D-DA): the declination angle in three dimensional space.

- The splaying angle: The angle between the shaft of the metatarsial and the vertical plane through the second metatarsal.

- The intermetatarsal angle (IMA): the angle between the shafts of two metatarsals. We distinguished two kinds:

- the measured intermetatarsal angle (measured-IMA): the simulated IMA as on a standard AP radiograph of the foot. 
- the three-dimensional intermetatarsal angle (3D-IMA): the three-dimensionall angle between two spatially oriented metatarsal shafts.

- Supination: the angle between the plane containing the two vectors of the metatarsals involved and the supporting plane; pronation is defined as the opposite of supination.

- Distortion: the difference between the measured angle and its corresponding three-dimensional angle.

\section{Simulation of radiography}

The basis of the mathematical simulation of radiography was the projection of an arbitrary point in space on the plane of the roentgen film ( $X$-ray projection imaging'). An algorithm calculated the point of intersection between the line through the focus of the roentgen tube and an arbitrary point in space with the plain of the roentgen film. In this simulation a metatarsal was represented by a vector. A vector can be characterized by its origin and its endpoint, and its projection is obtained by projecting the origin and the endpoint of the vector on the plain of the roentgen film.

\section{Theoretical assessment}

A portion of the size of a measured metatarsal angle originates from distortion. To illustrate the contribution of the distortion to the outcome of angle measurement, we calculated the distortion as a function of the measured angles on the lateral and the AP radiographs.

\section{Lateral radiograph}

The measured metatarsal declination angle (measured-DA) is the projection of the 3D declination angle (3D-DA). The measured size depends on both the size of the 3D-DA and the splaying angle. We constructed a mathematical model that simulated the standard technique of obtaining a lateral radiograph. With this model we calculated the distortion of the declination angle (Fig. 1A). Of the measured-DAs of 10, 20 and $30^{\circ}$ (angle 1), the corresponding 3D-DAs (angle 2) were calculated for a range of splaying angles (angle 3). Finally, the distortion (angle 1 - angle 2 , in ${ }^{\circ}$ ) was plotted as a function of the splaying angle (angle 3) (Fig. 1B).
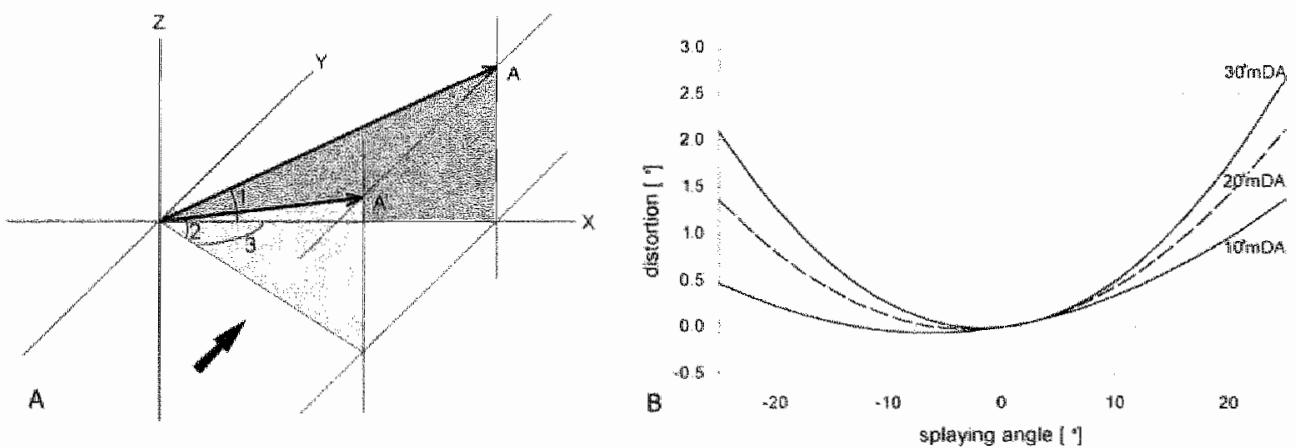

Fig. 1: In the mathematical model (A), the 30 dedination angle (angle 2) was callutated as anction of the splaying angle (angle 3) for several measured declination arigles (angle 1). The thick black arrow points in the direction of the central ray of the roentgen tube, perpendialar to the $x-z$ phane the plane of the roentgen illm, in dark grey). The corresponding curves (B) stwow the distortion (the difterence of the measured-DA and the 30.DA, angle 1 -angle 2) as a function of the splawing angle the curves were constructed for the measured-DA of 10 , 20 , and $30^{\circ}\left(10,20\right.$, and $\left.30^{\circ} \mathrm{mDA}\right)$. 


\section{AP radiograph}

On the AP radiograph the intermetatarsal angle (measured-IMA) is the projection of the 3D-IMA. Its actual size depends on the size of the 3D-IMA, the 3D-DA of both metatarsals, and supination-pronation ". To illustrate the distortion, we constructed two mathematical models (Fig. 2A and 3A) to calculate the measured-IMA and the 3D-IMAs. The geometry of these models was in accordance with the standard AP radiograph. In one model (Fig. $2 \mathrm{~A}$ ) the 3D-IMAs (angle 2, $\mathrm{A}^{\prime} O \mathrm{~B}^{\prime}$ ) were derived from the measured-IMA (angle $1, A O B$ ) with varying declination angles (angle $3, \mathrm{BOB}$ ). In the other model (Fig. $3 \mathrm{~A}$ ) we used varying supination and pronation (angle $3, \mathrm{ABA}^{\prime}$ ), In both models the calculations were carried out with a measured-IMA of 10,20 and $30^{\circ}$. Finally, the distortions (angle 1 - angle 2, in ${ }^{\circ}$ ) were plotted as a function of the declination and supination angle, respectively (Fig. 2B and 3B).

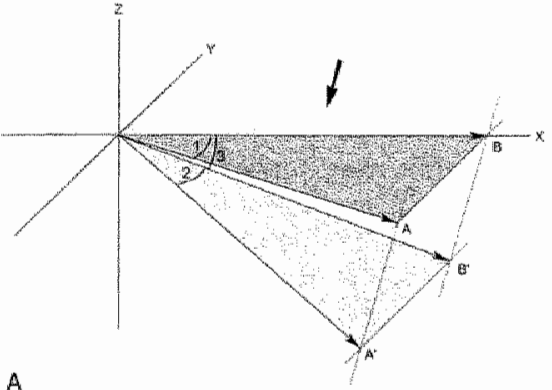

A

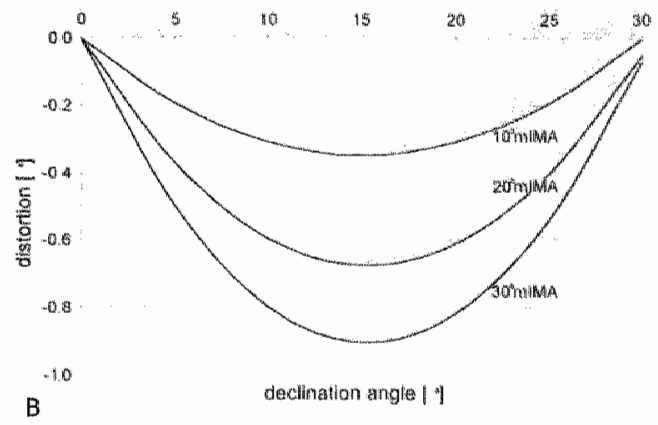

Fig. 2: In the mathematical model (A), the 3D-IMA (angle $A O B$, angle 2) was calculated as a function of the dedination angle (angle BOB', angle 3) for several measured IMAs (angle AOB, angle 1). The thick black arrow indicates the central ray of the $15^{\circ}$ craniocowdal angled beam. The plane of the roentgen fillm is deplcted in dark grey. The comesponding curves $(B)$ show the distortion (the difference of the measured IMA and the $3 D$ IMA, angle 1 - angle 2) as a function of the declination angle. The curves were constructed for the neasured-1MAs of 10,20, and $30^{\circ}\left(10,20\right.$, and $\left.30^{\circ} \mathrm{mim}\right)$.
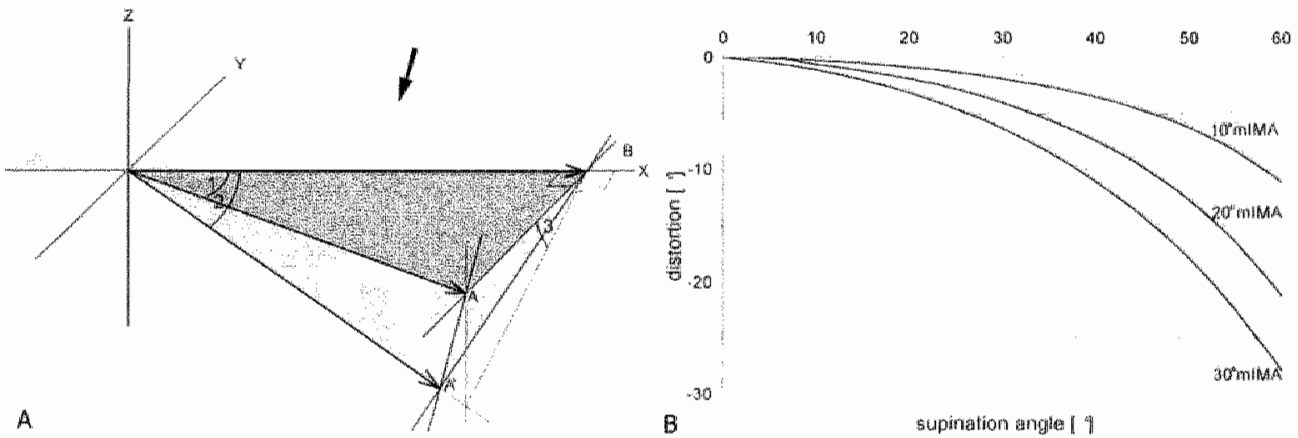

Fig. 3: In the mathematical model (A), the 3D-IMA (angle AOB, angle 2) was calculated as a functon of the supination angle (ABA, angle 3) for several measured-1MAs (angle AOB, angle 1). The thick biack arrow indicates the central ray of the $15^{\circ}$ cranio-catdal angled beam. The plane of the roentger firr is depoled in dak grey. The corresponding curves $(B)$ show the distortion (the difererce of the measured IMA and the $30-1 M$, angle 1 - angle 2) as a function of the supination angle. The cures were constructed for the measured-ilms of 40 , 20, arid $30^{\circ}$ $\left(10,20\right.$, and $30^{\circ} \mathrm{mm}(\mathrm{MA})$. 
In all three mathematical models, the range of values of the variables, splaying angle, declination angle, and supination angle (as indicated on the x-axes of Fig. $1 \mathrm{~B}_{r} 2 \mathrm{~B}$, and $3 \mathrm{~B}$ ), was in accordance with the range of values in the 10 normal volunteers.

\section{Clinical assessment}

To investigate how distortion caused by projection affects the angular measurements of the forefoot in daily practice, CT scans of the forefoot were obtained on 10 wolunteers. Our institutional review board approved this part of the study, and all volunteers gave informed consent. The 10 volunteers included: five men and five women (mean 34 years, range 19 to 51 years). They were healthy subjects, without foot complaints, calluses, deformities, or a history of previous trauma or surgery.

During the experiment the volunteers were standing upright on bare feet, with the right foot in front of the left, on the tabletop of a spiral CT scanner (Elscint, Oldelft, Delft, the Netherlands) with the gantry maximally tilted $\left(21.2^{\circ}\right)$. The right foot was positioned with the axis of the second metatarsal parallel to the lateral border of the tabletop. The spiral scan of the forefoot covered the area from first tarsometatarsal joint to the most distal interdigital web.

For each volunteer two consecutive scans of the right forefoot were made. During the first scan the volunteer placed the body weight on the left foot and gently laid the right foot on the tabletop: the non-weight bearing posture. Next, the volunteer loaded the right foot maximally during the scan: the weightbearing posture. Both scans of each volunteer were included because the position of the foot skeleton changes during loading ${ }^{15,16}$. The scan plane was defined as the $y-z$ plane, and the longitudinal direction of the tabletop as the x-axils.

To calculate the 3D position of the metatarsals, the centroid of the metatarsals was marked on each image, a centroid being the point with coordinates that are the average of the $y$ - and $z$-coordinates of all the pixels of that bone. We used the EasyScil 4.2.1 software package (Philips, Best, The Netherlands) for this semi-automated analysis. Knowing both the slice number and the thickness of the slices, an $x$-coordinate was added to the $y$ - and $z$-coordinates of each centroid. In analogy to the method of measurements on radiographs 7,10 , only the data relating to the shaft of the metatarsals were included, the data relating to the metatarsal head were discarded.

On these data two linear regression analyses were performed: one with the $y$-coordinate as the dependent and the $x$-coordinate as the independent variable and another with the $z$-coordinate dependent and the $x$-coordinate independent. These regression coefficients were the tangents of the angles of the metatarsal shaft with the $x-y$ and the $x-z$ planes, respectively. These angles determine the direction of the $3 \mathrm{D}$ vector of each metatarsal shaft. To test the reproducibility of this procedure, it was carried out twice. The mean difference between the first and the second calculation was $.23^{\circ}$ with a standard deviation of $.79^{\circ}$. Eighty-three percent of the differences we calculated were less than $1^{\circ}$. The average of the two measurements was used for further analysis. The data of the 3D vectors of the metatarsals were used in the simulation of the lateraland the AP radiographs.

\section{Lateral radiograph}

To determine distortion of the lateral radiograph, the differences of the measured-DA and the 3D-DA were calculated (Fig. 1A). First, the 3D-DA was calculated (angle 2, the angle between the 3D vector $O A^{\prime \prime}$ and the $x-y$ plane). Next, the 3D vector was projected on the $x-z$ plane (as if it were the roentgen film) in accordance with the standard technique of obtaining a lateral radiograph. Then, the measured-DA was calculated (angle 1 , the angle between the projected vector $O A$ and $x$-axis). Finally, the distortion (angle 1 - angle 2 , in ${ }^{\circ}$ ) was calculated. This procedure was followed for all metatarsals of all the volunteers in both postures. 


\section{AP radiograph}

To determine the distortion of the intermetatarsal angles (IMA) on the AP radiograph, the differences of the measured-IMA and the 3D-IMA were calculated (Fig. 4). First, the 3D-IMA was calculated (angle $1, A O B$, the IMA between the $3 D$ vectors of the two concerning metatarsals). Then both vectors were separately projected on the $x-y$ plane, and the measured-IMA was calculated (angle 2, $\mathrm{A}^{\prime} \mathrm{O}^{\prime} \mathrm{B}^{\prime}$, the angle between the two projected vectors). Finally, the distortion (angle 2 - angle 1, in ${ }^{\circ}$ ) was calculated. This procedure was followed for the IMAS 1-2, 2-3, 3-4, 4-5, and 1-5. The distortions were calculated for all the volunteers in both postures.

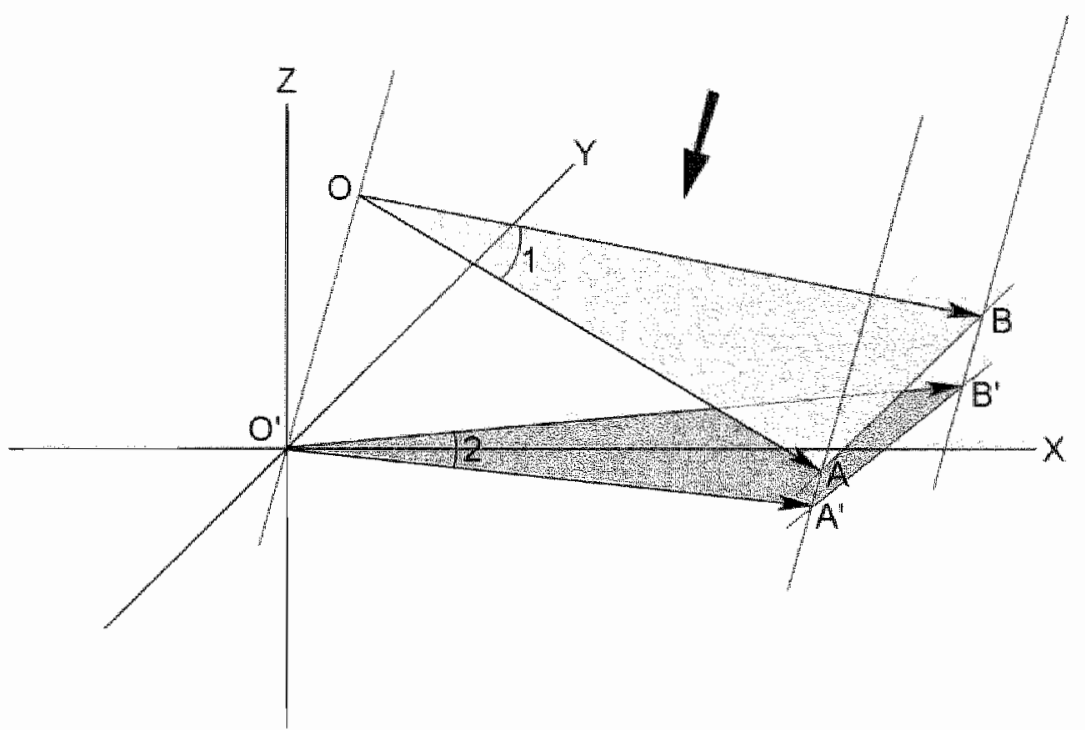

Fig. 4: Cakutation of the distortion of the IMA in nomal wolunteers. The 3D IMA (AOB, angle 1) was calculated from the wectors $O A$ and $O B$ that were derived from $C T$ data. The wedors were projected on the $x-y$ plane (the plane of the noentgen film, in dark grey) with 15 cranial-catdal abe angulation (thick black arrow). Next, the measured IMA (A'OE:, angle 2) was calculated from the projected vectors OA and OB: The difference of the measured-IMA and the 3Dm DWA (angle 2 - angle 1) was defined as distortion.

\section{Results}

\section{Declination angle}

Theoretically, the measured-DA overestimated the 3D-DA. Only at a measured-DA of $10^{\circ}$ was a minimal underestimation found. Larger measured-DAs, as well as larger splaying angles resulted in increasing distortions (Fig. 1B).

In the volunteers the measured-DAs overestimated the 3D-DAs up to $1.5^{\circ}$ and underestimated them up to $0.1^{\circ}$. The $2.5,10,90$, and 97.5 percentiles of the cumulative frequency distribution of the distortion are shown in Table 1 . The width of the $80 \%$-interval varied from $0.1^{\circ}$ for the fourth metatarsal to $0.8^{\circ}$ for the first metatarsal. The width of the $95 \%$-interval varied from $0.1^{\circ}$ for MT4 to $1.0^{\circ}$ for MT1. The overestimation is larger for the MT1 and MT5 than for the centrall metatarsals. 


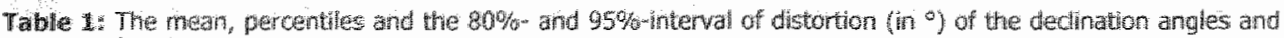

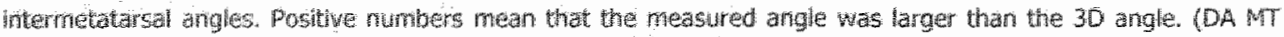

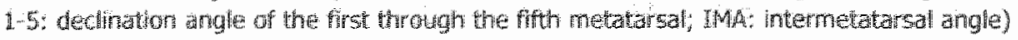

\begin{tabular}{|c|c|c|c|c|c|c|c|}
\hline & \multicolumn{5}{|c|}{ Percentiles } & \multicolumn{2}{|c|}{ Interval } \\
\hline & mean & 2.5 & 10 & 90 & 97.5 & $80 \%$ & $95 \%$ \\
\hline DAMT1 & 0.9 & 0.5 & 0.5 & 1.3 & 1.5 & 0,8 & 1.0 \\
\hline DAMT2 & 0.1 & 0.0 & 0.0 & 0.3 & 0.3 & 0.3 & 0.3 \\
\hline DA MT3 & 0.0 & 0.0 & 0.0 & 0.1 & 0.3 & 0.2 & 0.3 \\
\hline DAMT 4 & 0.0 & -0.1 & -0.1 & 0.0 & 0.1 & 0.1 & 0.1 \\
\hline DAMTS & 0.0 & -0.1 & -0.1 & 0.3 & 0.4 & 0.4 & 0.5 \\
\hline IMA 1-2 & -0.8 & -1.9 & -1.4 & -0.4 & -0.2 & 1.1 & 1.7 \\
\hline$M A-2-3$ & -2.6 & -5.2 & -4.3 & -1.0 & -0.9 & 3.3 & 4.3 \\
\hline TMA 3-4 & -3.0 & -4.8 & -4.6 & -1.5 & -1.4 & 3.1 & 3.4 \\
\hline IMA 4-5 & -1.1 & -2.3 & -21 & -0.3 & -0.3 & 1.7 & 2.0 \\
\hline IMA 1-5 & -2.8 & -4.8 & -4.2 & -1.5 & -1.2 & 2.7 & 3.6 \\
\hline
\end{tabular}

\section{Intermetatarsal angles}

Both theoretical models (Fig. 2A and 3A) showed that the measured-IMA underestimated the 3D-IMA as a function of the DA (Fig. 2B) and as a function of supination (Fig. 3B). The distortion associated with declination showed a maximum at $15^{\circ}$. It increased for larger measured-IMAs (Fig. 2B). The distortion associated with supination increased with larger supination and measured-IMAs (Fig. 3B).

In our volunteers the measured-IMAs underestimated the 3D-IM angles up to $5.4^{\circ}$. The 2.5 , 10,90 , and $97.5 \%$ percentiles of cumulative frequency distribution of the distortion are shown in Table 1 . The width of the $80 \%$-interval varied from $1.1^{\circ}$ for IMA $1-2$ to $3.3^{\circ}$ for IMA 2-3. The width of $95 \%$-interval varied from $1.7^{\circ}$ for IMA $1-2$ to $4.3^{\circ}$ for IMA $2-3$.

\section{Discussion}

Many sources of error in the angular measurements on the AP and lateral radiographs of the foot have been reported. Most attention has been paid to the assessment of the inter- and intrai-individual variations in various methods of measurement $3,4,9,11,17$. Additional sources of error are related to the relationship of the roentgen tube, the object, and the fillm such as tube angulation, focus-film distance ${ }^{10}$, and pronation or supination of the foot ${ }^{6}$. We focused on the distortion of metatarsal angles as measured on standard AP and lateral radiographs of the forefoot of normal volunteers, because it is relevant to clinical angular measurements.

Theoretically, the declination angle as measured on the lateral radiograph will overestimate the 3D declination (Fig. 1B). The distortion increased as the angle between the metatarsal and the plane of the film increased. This was in accordance with the results of the clinical assessment: the distortions at MT1 and MT5 were larger than those at the central metatarsals because MT2 parallels the plane of the film in the standard technique for obtaining a standing lateral radiograph of the foot. In our normal volunteers the maximum distortion was $1.5^{\circ}$.

on the lateral radiograph especially the first metatarsal declination angle is clinically relevant. It indicates the height of the medial longitudinal arch and is reported to be a relevant parameter when evaluating pathology at the furst metatarsophalangeal joint $11,13,18$. Compared to the normal declination angle of approximately $21^{\circ} 1,18,19$, the distortion in our normal volunteers was less than $1.5^{\circ}$. According to the theoretical model, even in a patient 
with a severely splayed forefoot the distortion was limited and therefore does not need to be considered in interpreting the values of the declination angles.

The intermetatarsal angle as measured on the AP radiograph is the result of interaction between the 3D-IMA, the declination of the metatarsals, and the supination-pronation. Theoretically, the measured-IMA underestimated the 3D-IMA. The distortion increased as the size of the measured-IMA increased. The clinical assessment also showed underestimation, up to $5.4^{\circ}$.

Of all intermetatarsal angles, IMA $1-2$ is most frequently used ${ }^{3}$. It has special importance in the clinical assessment of hallux walgus ${ }^{1}$. Small mangins are used to classify normal (less than $9^{\circ}$ ), from mild ( 9 to $11^{\circ}$ ) to even moderate hallux valgus (11 to $\left.16^{\circ}\right)^{5}$, although different cutoff points have been reported ${ }^{13}, 17$. Because this classification affects the choice of surgical procedure ${ }^{1,13,18}$, an analysis of the sources of error in this measurement is important. Concerning interobserver wariability, it was reported that to capture $95 \%$ of the differences between two observers an interval of 4 to $5^{\circ}$ was needed ${ }^{3-5}$. With a difference in angle of only $7^{\circ}$ between the upper limit of normal and al severe deformity, this interobserver variability is worrisome. Concerning distortion due to projection, the $95 \%$-interval was only $1.7^{\circ}$.

The IMA 4-5 clinically is used in assessment of a splayed forefoot and of abnomal pronation ${ }^{13}$. The $95 \%$-interval of the interobserver differences for this intermetatarsal angle was $4^{\circ}$, which is marked compared to the normal value of 7 to $8^{\circ} 13^{2} 20$. The $95 \%$-interval of the distortion was $2.0^{\circ}$.

These two examples suggest that physicians should be cautious when interpreting the outcome of measurements of intermetatarsal angles. The variability of clistortion due to projection showed to be only about one third of the interobserver variability of the measurement of IMA on radiographs. Nevertheless, distortion as a source of error should not be ignored because interobserver error and distortion are independent entities, and might: have cumulative effects on the result of a measurement of an IMA. Moreover, our results were obtained in normal volunteers. In patients with foot deformities the angles usually are larger (except the declination angle in flatfoot). In these situations the theoretical models: predicted an increase in distortion.

The calculation of the IMA was made on the assumption that the vectors of shafts of the metatarsals involved were positioned in a single plane. In practice, this is not necessarily the case.

\section{References}

1. Karasick D and Wapner KL: Hallux walgus deformity: preoperative radiologic assessment. AJP Am $I$ Roentgenol 1990; 155:119-123.

2. Allen DM and Nunley JA, 2nd: Measurement of the first/second intermetatarsail anglle following proxintal oblique metatarsal osteotomy. Foot Ankle Int 2002; 23: 64-67.

3. Saltzman $\mathrm{CL}_{1}$ Brandser EA, Berbaum $\mathrm{KS}$, et al.: Reliability of standaro foot radiographic measurements. Foot Ankle Int 1994; 15:661-665.

4. Resch $S$, Ryd $L$, Stenstrom A, et all: Measuring hallux valgus: a comparison of conventional radiography and dinical paraneters with regard to measurement accuracy. Foot Ankle Irit 1995; 16: $267-270$.

5. Condon $F$, Kaliszer $M$, Conhyea $D$, et al.: The first intermetatarsal angle in hallux valgus:." an andilysis of measurement reliability and the error involved. Foot Ankle Int $2002 ; 23: 717-721$.

6. Hlavac HF" Differences in $x$-ray findings with varied positioning of the foot. 3 Am Podiatry Assoc 1967; 57 : $465-471$.

7. Schneider $W$ and Knahr $K$ : Metatarsophalangeal and intermetatarsall angle: different values and interpretation of postoperative results dependent on the technique of measurement. Foot Ankle Int 1998; 19: $532-536$. 
8. Bryant Ja comperisor of radiographic foot measurements taken in two different positions. J Arr Podut Med Assoc 2001; $91: 234-239$.

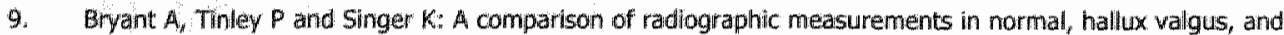
hallux umitus feet: J Font Anklle Surg 2000\% 39:39-43.

10. Venning $P$ and Hardy $k_{i}, H$. Sources of error on the production and measurement of standard radiographs of the foot: $\mathrm{Br}$ ] Radiol 1951; 24: 18-26.

11. Bryant A, Mahoney $\mathrm{B}$ and Tintey P: Lateral intermetatarsol angle: a usefiul measurement of metatarsus prithus elevatus? I Am Podiatir Med Assoc 2001; $91: 251$-254.

12. Camasta CA, Pontious $\mathrm{J}$ and Boyd RB: Quantifying magnification in pedal radiographs. $J$ Am Podiatr Med Assoc 1991: $81: 545-548$.

13. Kaschak TJ and Laine W: Surgical radiology, Clin Podiatr Med Surg 1988; 5: 797-829.

14. Smith RWW $_{\mathrm{N}}$ Regnolds JC and Stewart M: Hallux valgus assessment: report of research committee of American orthopaedic Foot and Ankle Society. Foot Ankle 1984; 5: 92-103.

15. Wejers 将E, Wienthamp $G H$, van Mameren $\mathrm{H}$, et al: The relationship of the position of the metatarsal heads and peak plantar pressure. Foot Ankle Int 2003; 24: 349-353.

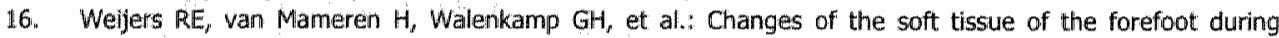
loading: a volumetric study. Foot $2003 ; 13: 70-75$.

17. Glasoe WM, Allen MK and Saltzman $\mathrm{CL}$; First ray dorsal mobility in relation to hallux valgus deformity and first intermetatarsal angle. Foot Ankle Int 2001; 22:98-10:.

18. Gentili $A_{r}$ Masih $S$, Yao $L$, et al.: Pictorial review: foot alxes and angles. Br J Radiol 1996; 69: 968-974.

19. Saraffian SK: Functional anatomy of the foot and ankle, in Anatomy of the Foot and Ankle, ed by Saraffian SK. JB Lippincott Company, Philadelphia: 1993. 47.4-602.

20. Tanaka $Y$, Takakura $Y$, Takaoka $T$, et all: Radiographic analysis of hallux vallgus in women on weightbearing and nonweightbearing. Clin Orthop 1997; 336: 186-194. 


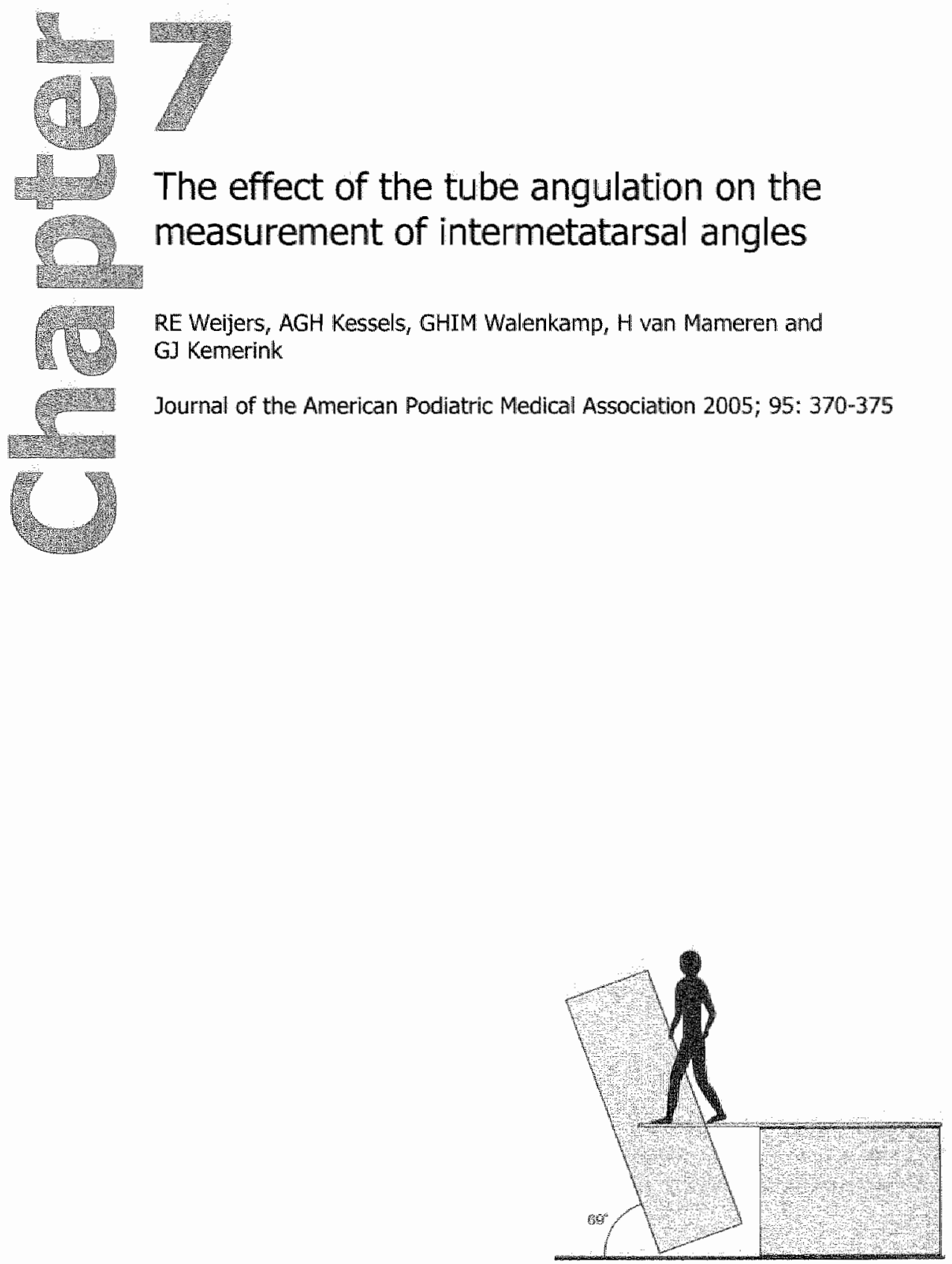




\section{Abstract}

We systematically investigated the effect of tube angulation on angular distortion for the antero-posterior radiograph of the foot. Three-dimensional data from the metatarsals originating from $C T$ scans of 10 healthy volunteers were projected on the supporting surface at various tube angulations to simulate radiography. The distortion of the intermetatarsal angles decreased from 1.20 to $3.5^{\circ}$ at $20^{\circ}$ tube angulation to 0.40 to 2.70 at $0 \%$ tube angulation.

The relatively small improvement in angular measurement using $0^{\circ}$ instead of $15 \circ$ tube angulation would not outweigh the adverse effect of changing the standard radiographic technique. Physician awareness of this source of error when planning surgical therapy seems more important. 


\section{Introduction}

In patients with foot deformities, radiographs are obtained as part of a clinical evaluation. on these radiographs, angular measurements are used to determine the severity of the deformation. In 1951, Venning and Hardy analysed sources of error in the production and measurement of radiographs of the foot. This publication illustrated the need for standardization of the radiograph. Since then, standardization of the anteroposterior (AP) or dorsoplantar view of the foot is widely advocated $2,3,4$. Despite this, various authors who described 'their' standard technique of the AP radiograph, used cranio-caudal tube angulations of $5^{\circ}, 15^{\circ}{ }^{3,6-10}$, or $20^{\circ}$. To our knowledge a systematic analysis of the relationship of tube angulation and the distortion due to the projection of the actual anatomy on the radiograph has not been performed. The goall of this study was to analyse this relationship using data from spiral computed tomographic (CT) scans of the forefoot of normall volunteers.

\section{Material and Methods}

Ten volunteers were included in the study: five men and five women (mean age, 34 years; age range, 19-51 years). The institutional review board of University Hospital Maastricht approved the study. All volunteers provided informed consent. They were healthy subjects, with no complaints of their feet, calluses, deformaties, or histories of previous trauma or surgery.

\section{Principle of the experiments}

The high contrast resolution of $\mathrm{CT}$ makes it an ideal modality to visualize bony structures in relation to their surrounding soft tissues. Therefore, the position and direction of the metatarsals in a scanned volume can be accurately determined. Using these data on spatial orientation of the metatarsals, conventional radiography can be simulated. The basis of the mathematical simulation of radiography was the projection of an arbitrary point in space on the plane of the radiographic film ('X-ray projection imaging'). An algorithm calculated the point of intersection of the line through the focus of the radiographic tube and an arbitrary point in space with the plane of the radiographic film. In this simulation, a vector represented a metatarsal. A vector can be characterized by its origin and its and point, and its projection is obtained by projecting the origin and the end point of the vector on the plane of the radiographic film.

\section{Imaging}

During the experiment, the volunteers stood upright on bare feet, with the right foot in front of the left, on the tabletop of a spiral CT scanner (Elscint; Oldelft, Delft, the Netherlands) with the gantry maximally tilted $\left(21.2^{\circ}\right)$. The right foot was positioned with the axis of the second metatarsal parallel to the lateral border of the tabletop. The spiral scan of the forefoot covered the area from the first tarsometatarsal joint to the most distal interdigital web.

For each volunteer, two consecutive scans of the right forefoot were performed. During the first scan, the volunteers placed their body weight on the left foot and gently laid the right foot on the tabletop (the nonweightbearing posture). Next, the volunteer loaded the right foot maximally during the scan (the weightbearing posture). Between the two scans, the sole of the foot did not lose contact with the tabletop. For technical details, we refer to previous publications $^{12,13}$. 


\section{Image processing}

To calculate the three dimensional (3D) position of the metatarsals, we first processed the images. On each image, the centroid of the metatarsals was noted, a centroid being the point with coordinates that are the average of the $y$ - and $z$-coordinates of all the pixels of that bone. We used the EasyScil 4.2.1 software package (Philips, Best, The Netherlands) for this semiautomated analysis. Knowing both the slice number and the thickness of the slices, an $x$-coordinate was added to the $y$ - and $z$-coordinates of each centroid. In analogy to the method of measurements on radiographs $1,6,10,11,14$, only the data relating to the shaft of the metatarsals were included; the data relating to the head were discarded.

On these data, two linear regression analyses were performed: one with the $y$-coordinate as the dependent variable and the $x$-coordinate as the independent variable and another with the $z$-coordinate as the dependent variable and the $x$-coordinate as the independent variable. These regression coefficients were the tangents of the angle of the metatarsal shaft with the $x-y$ and the $x-z$ planes respectively. From the two tangents, the 3D vector of each metatarsal shaft was calculated. To test the reproducibility, this procedure was carried out twice. The mean difference between the first and the second calculations was $.23^{\circ}$ with a SD of $.79^{\circ}$. Eighty-three percent of the differences we calculated were less than $1^{\circ}$. The average of the two measurements was used for further analysis.

\section{Definitions}

In this study, we used the following definitions:

- Anteroposterior or Dorsoplantar Radiograph: Radiograph of the foot of a standing individual using a central beam that is centred at the tarsometatarsal joint. The focus-film distance is $1 \mathrm{~m}^{4}$. In our simulations, we used a variable craniocaudal tube angulation in a sagittal plane $\left(0^{\circ}-20^{\circ}\right)$.

- Tube Angulation: The angle between the central ray of the radiographic tube and the longitudinal axis of the human body.

- Intermetatarsal Angle: the angle between the shafts of two metatarsals. We distinguished between two kinds:

1. measured intermetatarsal angle: the simulated intermetatarsal angle as could be measured on a standard anteroposterior radiograph of the foot; and

2. three-dimensional intermetatarsal angle: the 3D angle between two spatially oriented metatarsal shafts.

- Distortion: the difference the measured intermetatarsal angle and the 3D intermetatarsal angles (in degrees).

\section{Simulation of tube Angulation}

The optimal tube angulation was defined as the angulation that was associated with the smallest average distortion. To calculate the distortion, the following procedure was carried out (Fig. 1). First, the intermetatarsal angle between the 3D vectors of the two concerned metatarsàls was calculated (the 3D intermetatarsal angle, angle $1, A O B$ ). Next, the vectors were successively projected onto the $x-y$ plane (as if it were the radiographic film) with simulated tube angulations of $0^{\circ}, 5^{\circ}, 10^{\circ}, 15^{\circ}$, and $20^{\circ}$ (angle 3). Besides the varying tube angulation, the geometry of this projection was identical to the standard technique of an anteroposterior radiograph. Then the intermetatarsal angle between both projected vectors was calculated (the measured intermetatarsal angle, angle $2, A^{\prime} O^{\prime} B^{\prime}$ ). Finally, the distortion was calculated (angle 2 minus angle 1). This procedure was followed for intermetatarsal angles 1-2, 2-3, 3-4, 4-5, and 1-5 of all volunteers in both postures. We included the nonweightbearing and weightbearing data as two separate observations because loading of the foot changes the position of the metatarsals in relation to the central ray of the radiographic tube. 


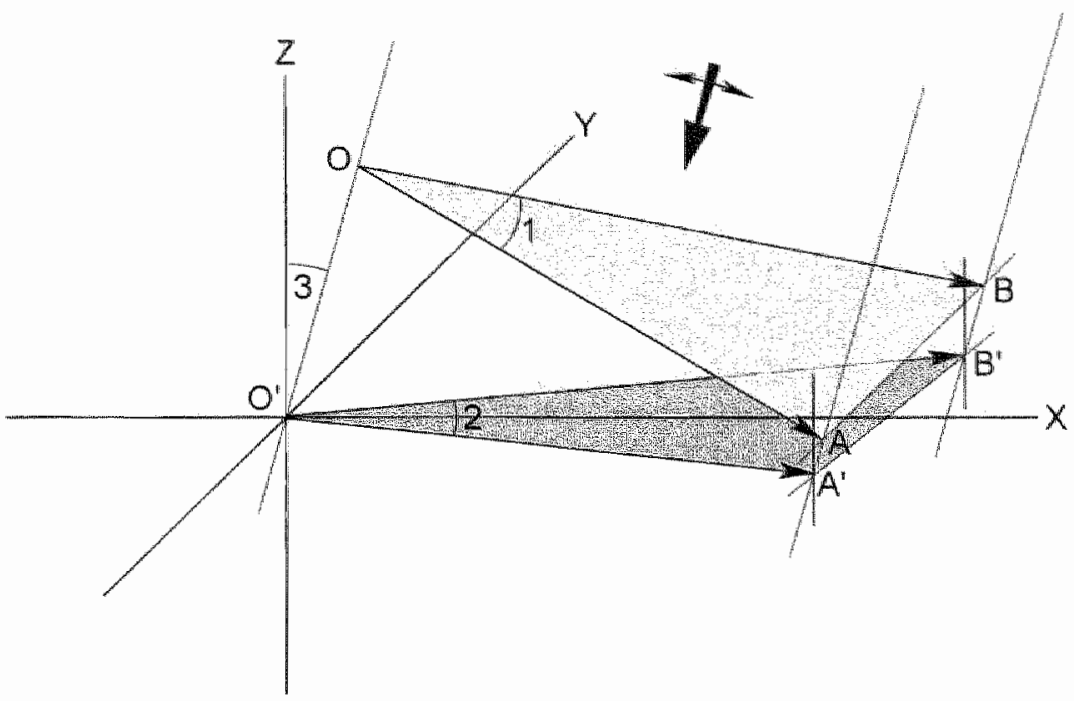

Fig. 1: Model of calculation. The metatarsals are represented as vectors. First, the angle between the two metatarsal vectors $D A$ and $O B$ (angle $1, A O B$ ) was calculated Next, both vectors were projected on the $x$ " $y$ plane

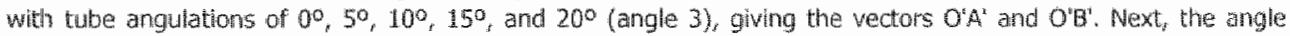
between the projected metatarsal vectors (angle $2, \mathrm{~A}^{\prime} \mathrm{O}^{\prime} \mathrm{B}^{\prime}$ ) was calculated. Finally, the distortion was calculated (angle 2 minus angle 1). The large black arrow indicates the variable craniocatudal tube angulation.

\section{Results}

Figure 2 shows the mean and the distribution of the distortion of the intermetatarsal angles at the various tube angulations. Generally speaking, the measured intermetatarsal angle underestimated the 3D intermetatarsal angle (measured angle $<3 \mathrm{D}$ angle). Onlly in $12 \%$ of the cases (31 of 250) was an overestimation observed at intermetatarsal angles 1-2, 4-5, and $1-5$, with a maximum of $0.9^{\circ}$. Increasing tube angulation gave fewer overestimations $\left(21,8\right.$, and 2 cases at $0^{\circ}, 5^{\circ}$, and $10^{\circ}$ tube angulation respectively) at the cost of larger underestimation.

As mentioned previously, measured intermetatarsal angles 1-2, 4-5, and 1-5 sometimes overestimated the $3 \mathrm{D}$ intermetatarsal angle (distortion $>0^{\circ}$ ) and sometimes underestimated the 3D intermetatarsal angle (distortion $<0^{\circ}$ ). In these cases, the mathematical mean underestimated the magnitude of distortion. Therefore, we also calculated the means of the absolute values of differences of the intermetatarsal angles (Fig. 3). The distortion at $0^{\circ}$ tube angulation increased in the following order: intermetatarsal angle $1-2<4-5<1-5<2-3<$ $3-4$ (range, $0.4^{\circ}-2.7^{\circ}$ ). As the tube angulation increased, the mean absolute distortions also increased. This effect was most pronounced for intermetatarsal angle $1-5$. At $20^{\circ}$ tube angulation, the distartion ranged from $1.2^{\circ}$ to $3.5^{\circ}$. 


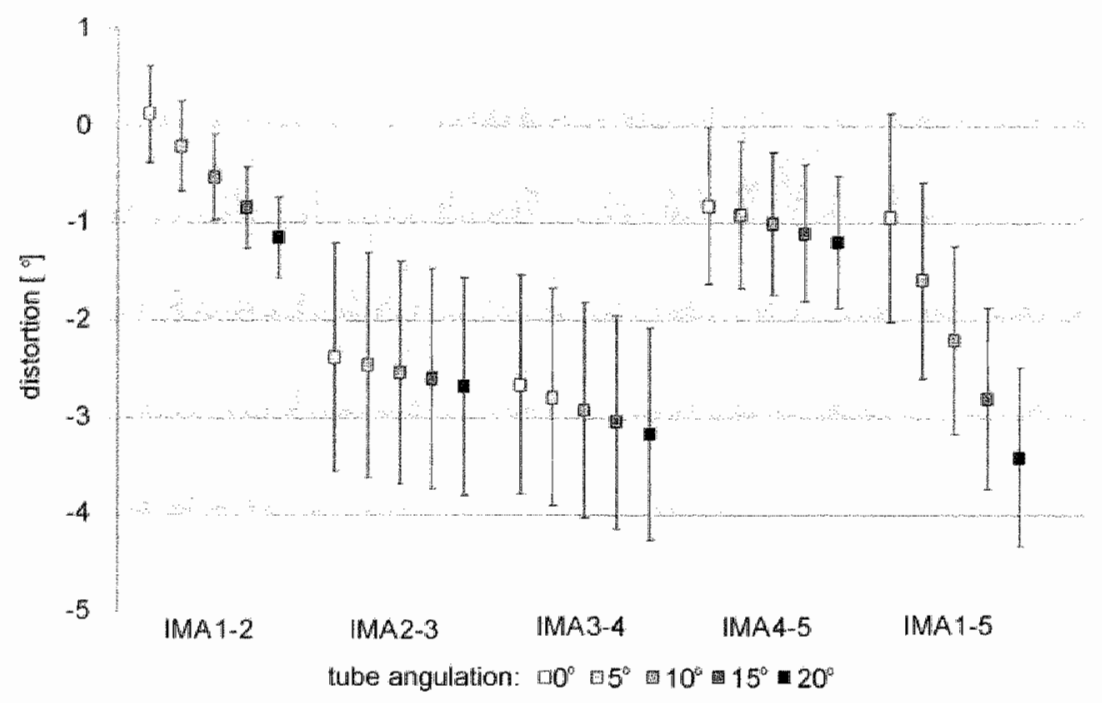

Filg. 2: Mean distortion of intermetatarsal angles (IMAs) $1-2,2-3,3-4,4-5$, and $1-5$ at $0^{\circ}, 50,10^{\circ}, 150$, and 200 tube angulation. A negatve number indicates that the measured angle undwestimated the three-dimensional intermetatarsal angle. Error bars represent $95 \%$ confidence intervals.

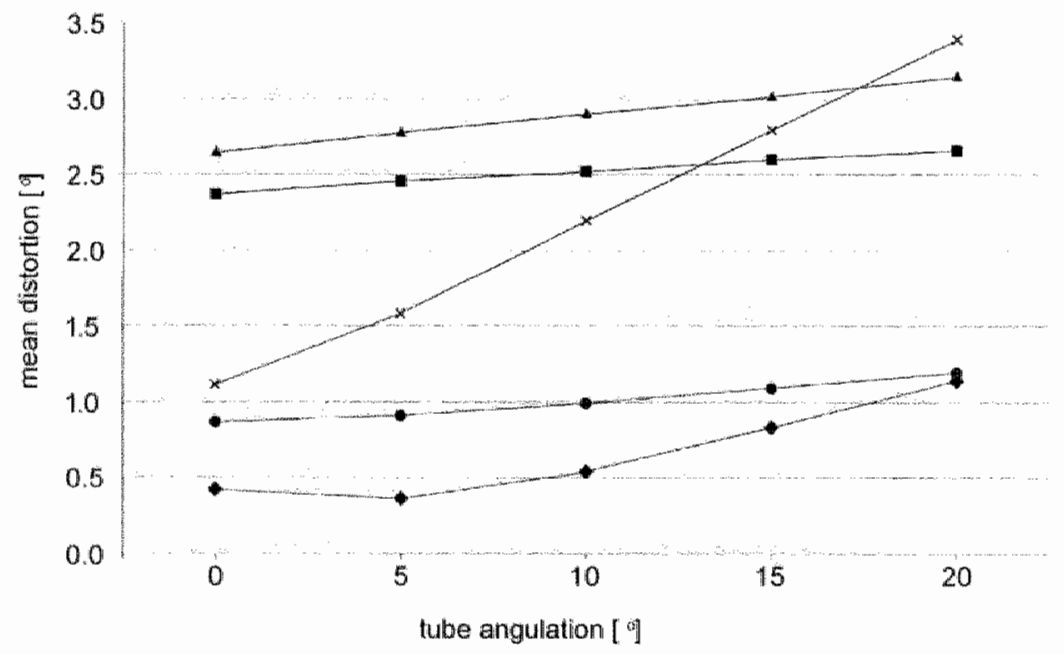

-IMA1-2 -IMA2-3 AMA3-4 -IMA4-5 XIMA1-5

Fig. 3i. The means of the absolute values of the distorions of intermetatarsal angles (MAS) $1-2,2-3,3-4,4-5$, and 1 - 5 at $00,5 \%, 100,150^{\circ}$ and $20^{\circ}$ thube angulation. 


\section{Discussion}

Most articles on the measurements of dorsoplantar radiographs report a $15^{\circ}$ or $20^{\circ}$ craniocaudal tube angulation. The American Orthopedic Foot and Ankle Society recommended a tube angulation of $15^{\circ}$ ". The length of the clinically most important first and second metatarsals would be minimally distorted at a tube angulation of $9^{\circ}$ to $15^{\circ}$ because the normal declination of these metatarsals are $18^{\circ}$ to $25^{\circ}$ and $18^{\circ}$, respectively ${ }^{15}$. Nevertheless, the metatarsal length as measured on radiographs overestimates the actual length by approximately $15 \%$ "Matters are different for angular measurements: the intermetatarsal angles to be measured not only depend on the tube angulation and declination of the metatarsals involved but also on the 3D intermetatarsal angle and the pronation/supination ${ }^{3}$. This makes the choice of an optimal tube angulation less obvious. In this study, we focused on the distorting effects of tube angulation on the angular measurements, not the linear ones.

Generally speaking, we found that the distortion of intermetatarsal angles was at a minimum when the radiograph was made without tube angulation. Even on the radiograph made without angulation, however, the intermetatarsal angle as measured by an observer underestimated the actual (3D) intermetatarsall angle. Overall, the underestimation increased from $-0.1^{\circ}$ to $2.7^{\circ}$ at $0^{\circ}$ tube angulation to $1.2^{\circ}$ to $3.5^{\circ}$ at $20^{\circ}$ tube angulation. This effect was most prominent for intermetatarsal angle $1-5$, which was to be expected because this is the largest of all the intermetatarsal angles.

Intermetatarsal angle $1-2$ is clinically the most relevant. The mean distortion of this intermetatarsal angle increased from $-0.1^{\circ}$ to slightly more than $1^{\circ}$ at $20^{\circ}$ tube angulation. Whether this is important depends on the point of view. Considering that the difference of the upper limit of normal and the lower limit of a severe deformity of hallux valgus is only $7^{\circ}$ ${ }^{17}$, the error is considerable. Compared with the reported interrater variations of the measurement itself, where one observer should be within $4^{\circ}$ of another observer $95 \%$ of the time ${ }^{11}$, the effect is relatively small.

In light of these findings, it seems desirable to make a standard anteroposterior radiograph of the foot without tube angulation. However, this would imply that first and second metatarsals would foreshorten on the radiograph because those metatarsals normally show the largest declination angle. Moreover, weightbearing during the radiographic examination is recommended to document maximum deformity ${ }^{3,4,6}$. A normal standing position of the patient during imaging makes it impossible to obtain a radiograph without tube angulation, because the pelvis/abdomen would obstruct the radiographic tube. Finally, almost all data that have been published, originated from radiographs that were made with $15^{\circ}$ or $20^{\circ}$ tube angulation. Using a different technique to make the radiographs would hamper comparison of these angular measurements with those of previous publications and thus would hinder clinical decision-making.

\section{Conclusion}

The tube angulation of an anteroposterior radiograph affects the intermetatarsal angles. However, the relatively small reduction in the distortion of the intermetatarsal angles would not outweigh the adverse effects of changing the standard positioning technique. Physician awareness of this source of error when planning surgical therapy seems to be more important than a change in technique. 


\section{References}

1. Menning $\mathrm{P}$ and Hardy $\mathrm{R}_{t} \mathrm{H}_{*}$ Sources of error on the production and measurement of standard radiographs of the foot. Br J Rediol 1951; 24: 18-26.

2. Stevens PM: Radicgraphic distortion of bones: a marker study. Onthopedics 1989; 12: 1457-1463.

3. Havac HF: Diferences in tay findings whith varied positioning of the foot. 3 Am Podiatry Assoc 1967; 57 : 4654711 .

4. Smith RW, Reynofds IC and Stewart M. Hallux valgus assessment: report of research committee of American Orthopardic Foot anid Arkkle Society. Foot Ankle 1984; 5: 92-103.

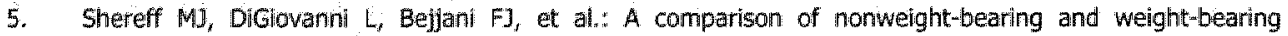
radiagraphs of the foot. Foot Ankle $1990 ; 10: 306-311$.

6. Kaschak TJ and Laine W: Surgical radiology. Clin Podiatr Med Surg 1988; 5: 797-829.

7. Schneider $W$ and Knahr Ko: Metatarsophalangeal and intermetatarsal angle: different values and interpretation of postoperative results dependent on the technique of measurement. Foot Ankle Int 1998; 19: $532-536$.

8. Bryant $A$, Tinley $P$ and Singer $K$ : $A$ comparison of radiographic measurements in normal, hallux valgus $s_{p}$ and hallitu limitus feet. J Foot Ankle Surg 2000; $39: 39-43$.

9. Karasick D and Wapner $\mathrm{KL}$ : Halluw walqus deformily: preoperative radiolagic assessment. Am J Roentgenol 1990; 155: 19-123.

10. Tamaka $Y$, Takakura $Y$, Takaoka $T$, et al.: Radiographic analysis of hallux vallgus in women on weightbearing and nonweightbearing. Clin Orthop 1997; 186 194 .

11. Saltaman $\mathrm{CL}$, Brandser EA, Berbaum $\mathrm{KS}$, et all: Reliability of standard foot radiographic measurements. Foot Ankle lint 1994; 15: 661-665.

12. Weijers RE, Wallenkamp $G H$, van Mameren $H$, et al: The relationship of the position of the metatarsal heads and peak plantar pressure. Foot Ankle Int 2003; 24: 349-353.

13. Weijens RE, van Mameren $H$, Walenkamp $\mathrm{GH}_{\text {, }}$ et al.: Changes of the soft tissue of the forefoot during loading: a volumetric study. Foot 2003; $13: 70-75$.

14. Gentill A, Masih S, Yao L, et al.: Pictorial review: foot axes and angles. Br I Radiol 1996; 69: 968-974.

15. Saraffian SK: Functional anatomy of the foot and ankle, in Anatomy of the Foot and Ankle, ed by Saraffian SKK. Lippincot J.B., Philadelphiti; 1993, 532-534.

16. Camasta CA, Pontious J and Boyd RB: Quantifying magnification in pedal radiographs. I Am Podiatr Med Assoc 1991; 81: 545-548.

17. Condon $F$, Kaliszer $M$, Conhyea $D$, et all: The first intermetatarsal angle in hallux valgus: an analysis of measurement reliability and the error involved. Foot Ankle Int 2002; 23: 717-721. 


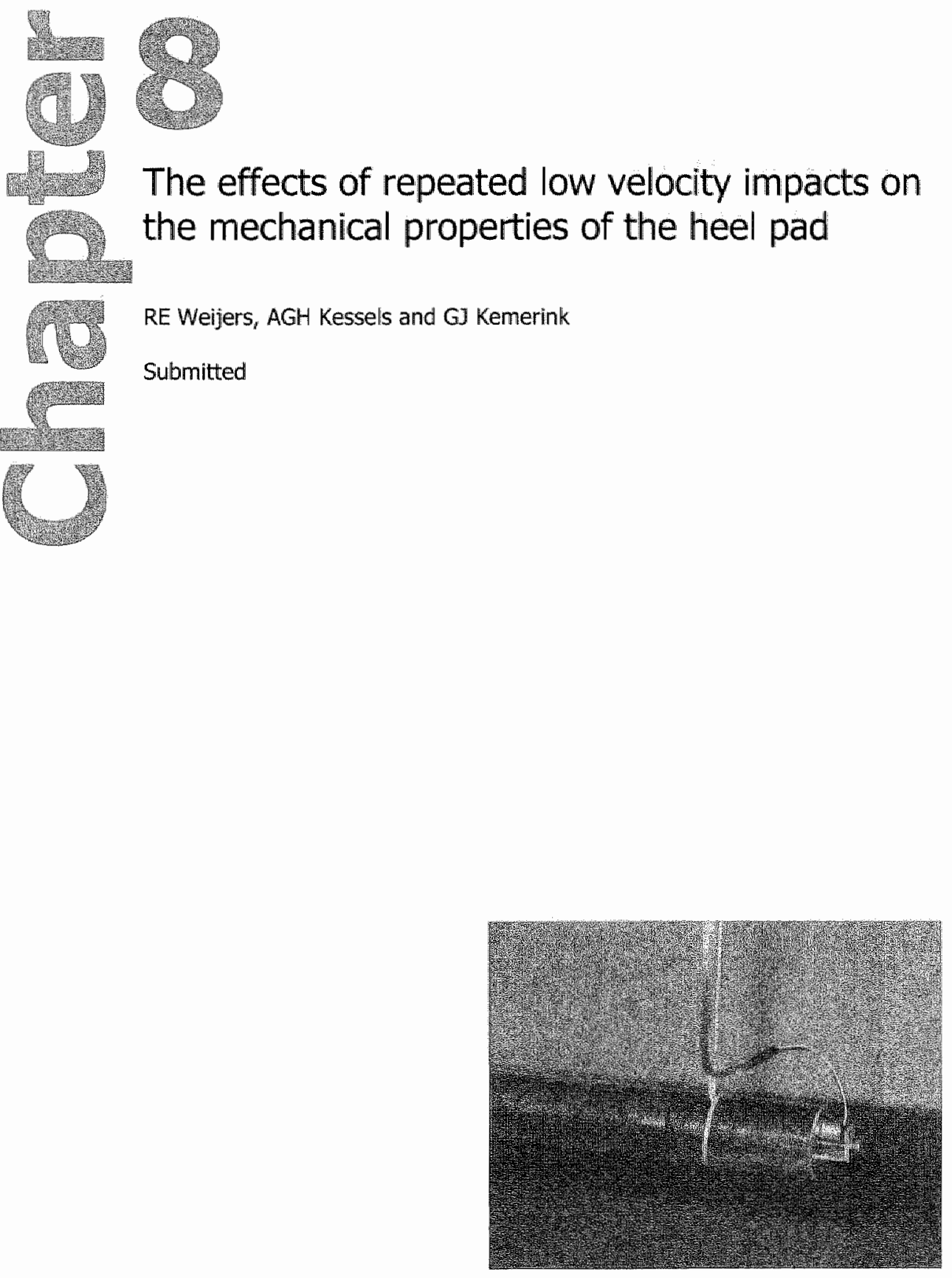




\section{Abstract}

The heel pad is said to be the most important damper of the impact force, but its behaviour is still not fully understood. In vitro examinations on isolated heel pads with a material-testing machine showed that the heel pad became more resilient after repeated impact at loading tates comparable to walking. On the other hand, in vivo examinations on lower legs with repeated pendulum impacts failed to show changes in the mechanical properties at impact speeds pertained to running. To contribute to the understanding of the mechanical properties of the heel pad and its relation to the lower leg, we simulated the impact force of wallking in vivo by means of a pendulum impact test. The pendulum consisted of a suspended iron bar that was instrumented with a mono-axial accelerometer. Only normal forces were measured. Eleven healthy male subjects were subjected to impacts with a velocity of $0.2,0.4$, and $0.6 \mathrm{~m} / \mathrm{s}$. To detect changes with increasing number of impact, sequences of 18 impacts were acquired. Of each impact the following parameters were considered: (1) the dissipated energy, (ii) the maximum force, (iii) the stiffness of the soft tissue and (iv) the maximum indentation. To the 18 values of each parameter in a sequence, an exponential function was fitted. From this function, the relative change of the parameters between the first and the last impact of a series was calculated. Repeated impacts resulted in a rise of the maximum force and the maximum indentation and a decline of the dissipated energy and the stiffness (all in the order of $3 \%$ ). If a parameter showed changes in value, most of the change was alleady reached after the very first impacts. At higher impact velocities, consecutive impacts showed a gradual fall of the maximum force and stiffness that could not be explained from the visco-elastic properties of the heel pad. We suggested that these observations expressed the adaptation of the muscles of the calf to the impact force, also referred to as 'tuning'. We conclude that the damping effect of the lower leg more than compensated the loss of damping power of the heel pad on repeated impacts. These observations are of theoretical interest and may increase the insight in the physlology of locomation. 


\section{Introduction}

The heel pad is said to be an important damper of the impact force during the landing phase of locomotion, the heel strike ${ }^{{ }^{1} 2}{ }^{2}$. The question was whether repeated impacts affected its damping power (in this context defined as the ability to decrease an impact force). During the heelstrike, the heel pad becomes flatter and wider. With this deformation, the heel pad absorbs part of the energy of the impact. At heel rise, the heel pad can recover from the deformation. If the heel pad would behave completely elastic, all the stored energy would be returned, and the heel pad would regain its original shape. However, the soft tissue of the heel pad combines elastic and viscous properties ${ }^{3}$. Therefore, at heel rise only part of the energy that was absorbed during heelstrike will be returned (the elastic property), the rest. will be dissipated (the viscous property). Eventually, the heel pad will recover from the deformation, but as the rest time (i.e. between toe-off and swing phase) pertained to walking is $0.5 \mathrm{sec}^{4}$, the pad is far from relaxed ". In the next gait cycle with the heel pad already flattened, the ability of the heel pad to dissipate energy will diminish. In vitro studies on isolated heel pads confirmed the decrease in energy dissipation on repeated loadings ${ }^{5,6}$. However, energy dissipation merely expresses the relation between the energy input at: (simulated) heelstrike and the energy output at (simulated) heel-off. It does not give information on how the decrease is attained or on a possible loss of damping power on repeated impacts.

In a material testing machine, the actuator of the machine imposes a fixed sinusoidal indentation (described by amplitude and frequency) in an isolated heel pad. The related force is recorded. As the heel pad changes on cyclic loading, the energy input necessary to reach the fixed indentation will also change. The results are therefore difficult to translate in terms of loss of damping power. At heelstrike, a certain amount of energy is transferred to the ground through the heel pad. Therefore, to simulate the heel strike, we prefer pendulum impact testing because not the indentation but the amount of kinetic energy of the impact is chosen. By a double integration of the acceleration signal during impact, a load-compression curve was constructed. It gives the dissipated energy. Repeated impacts give information on the theoretically expected decrease of the dissipated energy and on a possibly associated decrease in damping power. However, in vivo pendulum impact tests failed to show any effect of repeated loadings 2,7 . These studies used impact velocities that are mostly observed during running (about $1.0 \mathrm{~m} / \mathrm{s}$ ). From an evolutionary point of view, it is more likely that the biomechanical properties of the human body are tuned in to the lower impact velocities of walking. Therefore, the goal of our study was to imvestigate the expected decrease in energy dissipation on repeated impacts using pendulum impact testing at lower impact velocities, and if present, how the decrease is attained. Moreover, to our knowledge, presently hardly any in vivo data are available at the lower impact velocities of walking. The results are of theoretical interest and may increase insight in mechanical properties of the heel pad at lower impact velocities.

\section{Material and methods}

Impact tests with a pendulum can measure the mechanical properties of heel pads. To answer the question whether the mechanical properties of heel pads change after repeated loadings, we studied sequential impacts of an instrumented pendulum on heel pads in vivo. To analyse velocity dependence, we conducted three series of sequential impacts. Each series had a different starting amplitude of the pendulum. 


\section{Pendulum}

The pendulum consisted of a cylindrical steel bar suspended by four thin inextensible synthetic cords in a stainless steel frame with an adjustable height. By using two pairs of cords, instead of simply two single ones, transwerse oscillations were largely suppressed. The pivot points of a pair of cords on the frame were $30 \mathrm{~cm}$ apart in the lateral direction. The effective length of the suspending cords was $1.5025 \mathrm{~m}$. The bar had a length of $67.0 \mathrm{~cm}$, a cross section of $9.62 \mathrm{~cm}^{2}$ and a mass of $5.1175 \mathrm{~kg}$. The free swing period was $2.46 \mathrm{~s}$.

The steel bar was instrumented with a band-pass mono-axial accelerometer (model PB3ACW, Oceana Sensor, Virginia Beach, WA) with a nominal sensitivity of $100 \mathrm{mV} / \mathrm{g}$, with $\mathrm{g}$ the gravitational constant. The frequency response was within $\pm 5 \%$ from $1 \mathrm{~Hz}$ to $4 \mathrm{kHz}$. A multifunction PC board (AT-MIO-16E-10, National Instruments, Austin, TX) digitised the signal of the accelerometer. This board had an adjustable gain amplifier and a 12-bit analog to digital converter. The sample rate during data acquisition was $5 \mathrm{kHz}$; the duration of the sampling period was 5 seconds. The signal of the actual measurements on human heels had to be corrected for the (single pole) response fall-off of the accelerometer at low frequencies; this was done in the frequency domain. Corrections to be applied due to high frequency fall-off were negligible. For data correction (low-off compensation) and data analysis, we used Matlab wersion 6.1 (The MathWorks Inc., Natick, MA, USA). For a detailed description of the calibration of the accelerometer, the compensation for the low-frequency fall-off, and the process of calculation the force, velocity and position of the pendulum, we refer to a previous publication ${ }^{8}$.

\section{Subjects}

The subjects were healthy males, without physical complaints of their feet. No plantar callus formation was present. Eleven subjects were included (mean age: 38.1 years, range 29-54; mean weight: $79.1 \mathrm{~kg}$, range 70-89; mean length: $1.82 \mathrm{~m}$; range $1.74-1.93)$. The Institutional Ethic Committee approved the study. All subjects gave informed consent.

\section{Subjects and pendulum position}

of all subject the left heel pad was investigated. The subjects lay supine on the ground beside a massive rectangular concrete pillar with a bare left leg. The hip was flexed a little over $90^{\circ}$ and slightly abducted; the knee was flexed a little over $90^{\circ}$. Thus, the subjects had to place the knee against the flat surface of the pillar with the lower leg in horizontal position.

Because we wanted to measure the damping properties of the heel, we had to minimize unwanted additional damping of the soft tissue over the knee. The wall only supported the lateral femoral condyle because not only the skin but also the quadriceps muscle covered the medial condyle. To reduce the unwanted damping furthermore and at the same time minimize axial translation of the lower leg during pendulum impacts, we preloaded the lower leg with $70 \mathrm{~N}$ in the axial direction. For this purpose, we used a 'stirrup' with the straps fixed to the pillar. With an interposed spring based weighing device, we adjusted and monitored the load with which the lower leg was axially "sandwiched" between the stirrup and the wall (Fig. 1). A removable plaster cast served as a stirrup and covered the foot and the distal lower leg with the exception of the plantar side of the heel.

To secure the pasition and enable the subject to relax throughout the experiment, the leg with the stirrup was placed in a homemade 'vice', which was mainly constructed out of wood. The rubber jaws of the vice compressed the lower leg over the imedial and lateral side of the tibia plateau and the malleoli (Fig. 1). A separate block of wood supported the heel at the insertion of the Achilles tendon to secure a horizontal position of the leg throughout the experiment without confining the sole at the posterior side.

Prior to the experiment, the skin over the tuber calcanei was marked under ultrasound guidance. The mark served as the centre of the impact region. First, we leveled the centre of 
the bar with the mark on the heel by adjusting the height of the bearing frame. Next, we aligned the bar with the axis of the tibial bone. In equilibrium, the centre of the end of the bar just touched the marked skin of the heel through the opening in the plaster (Fig. 1). Finally, the frame was clamped between two walls to prevent oscillations of the frame. To monitor the position of the leg during the course of the experiment, the circumference of the touching bar was marked on the skin. Although we put considerable effort into stabilizing the leg and foot during the experiment, some degree of movement during impact was unavoidable, especially in the axial direction of the leg.

\section{Measurement protocol}

We measured at three impacts velocities: $0.2,0.4$ and $0.6 \mathrm{~m} / \mathrm{s}$. The velocity of the impacting bar was determined by the difference in height (h) between the starting and the impact position (the change from potential in kinetic energy; velocity $=\sqrt{ }(2 \mathrm{gh})$, with $g=9.82 \mathrm{~m} / \mathrm{s}^{2}$ ). At each velocity, we measured a sequence of 18 limpacts over six minutes, at a rate of three impacts per minute. Between two sequences, we checked the alignment of the leg, foot and bar and adjusted the amplitude of the pendulum. This took about two minutes.

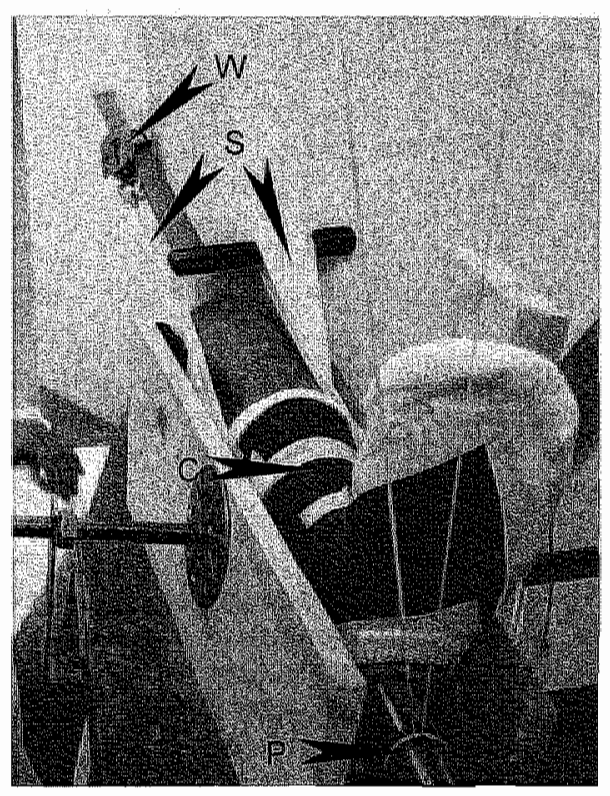

A

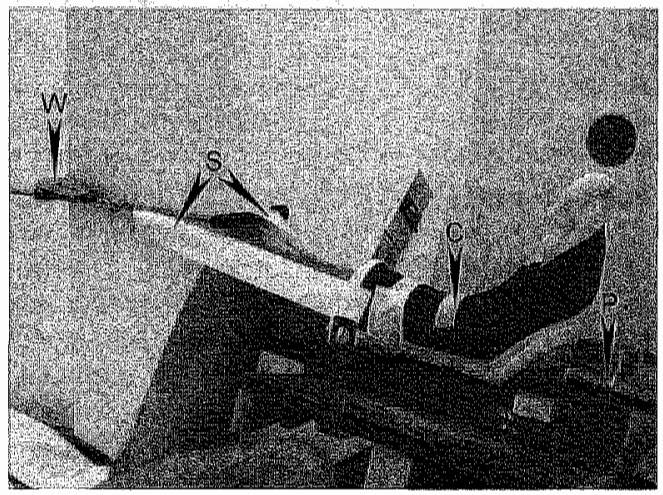

Fig. 1: A view from above (A) and atdelong wew (B) of the postion of the wolunteer and the pendulum duning the experiment. Wote the axial confinement of the lower leg between the conctete pilat and the casto for the

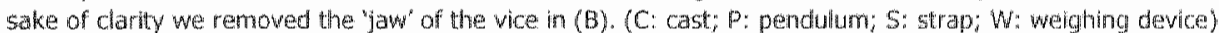

\section{Definition of the parameters}

By means of a double integration of the deceleration signal, a load-compression curve was constructed. Details of this procedure were previously described in Weijers, in press. From this curve, the following parameters were callculated (Fig. 2):

- $F_{\text {max }}$ : maximum Force ( $=$ mass of pendulum * maximum acceleration) [N]

- $D_{\text {max }}$ : maximum compression [mm]

- Stiffness $[\mathrm{kN} / \mathrm{m}]$ : the coefficient of the linear regression equation of the linear part of the load-compression curve. 
- Edis: dissipated energy: the lost energy expressed as a percentage of the kinetic energy of the pendulum at the beginning of the impact. It is defined as: (area under the load-compression curve during compression minus the area during recoll)*100/ area under the load-compression curve during compression.

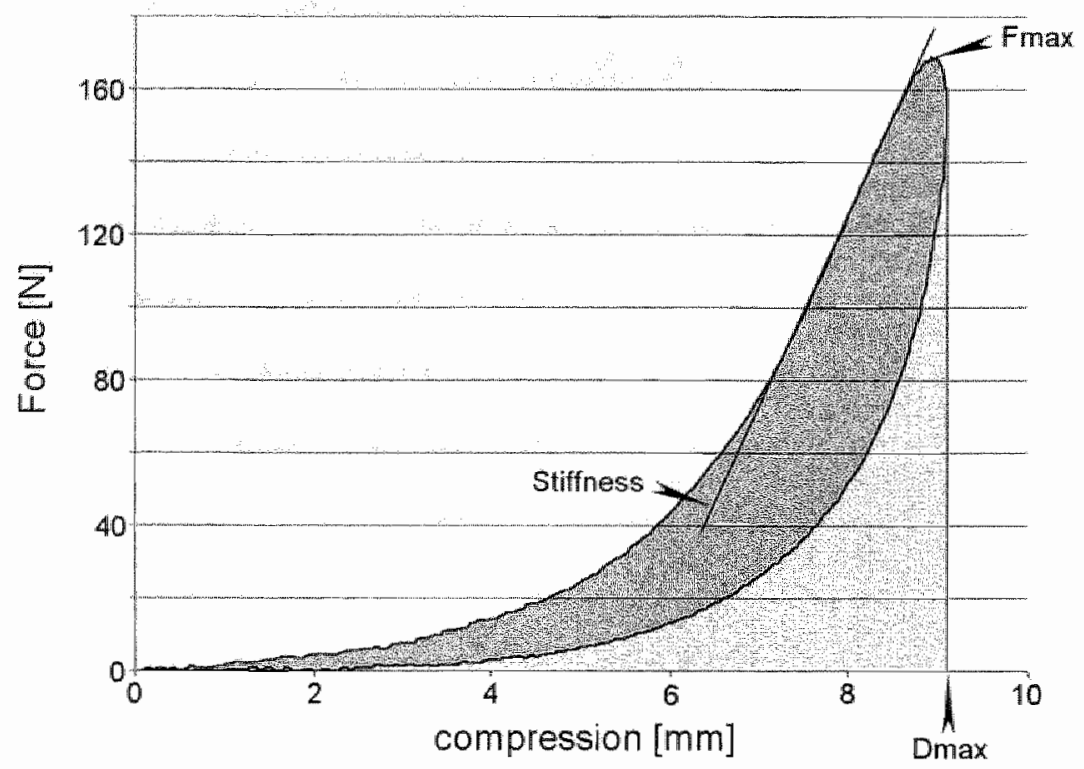

Fig: 2: An exantple of a load-compression curve of a volunteer at $0.4 \mathrm{~m} / \mathrm{s}$. The Fas was directly calculated from the acceleration data, the $\mathrm{D}_{\text {max }}$ Stiffness and $\mathrm{E}_{\text {dit }}$ wetre derived from the curve. The stiffress was deftied as the regression coefictent of the regression line over the linear part of the curve. The Eals was defined as: the dark grey area* $100 /$ (light ghey plus dark grey area).

\section{Statistical analysis}

At each velocity, we measured a sequence of 18 consecutive impacts. Four parameters that characterized a single impact were calculated. Thus, of each subject at each impact velocity, four series of sequential values were available. Previous reports on in vitro tests only showed a change in the magnitude of parameters at the first few impacts of a sequential loading ${ }^{5,6}$. To the series of sequential data of all parameters, we fitted an exponential function. The general form of this function was $f(n)=a+b^{*} e^{t^{*} n}$, with ' $n^{\prime}$ ' the $n^{\text {th }}$ impact of a sequence. Next, we calculated the relative change between the first and the $18^{\text {th }}$ impact: $(f(18)-f(1))^{*} 100 / f(1)$. Finally, these relative changes were statistically analysed with two tests. The two-tailled one sample t-test was used for each parameter at each velocity separately to test the null-hypothesis that there was no difference in magnitude of the parameter at the first and the $18^{\text {th }}$ impact. The repeated measure ANOVA-test was used to test whether there was a relation between a parameter and the impact velocities. We used SPSS 10 (SPSS Inc., Chicago, Illinois, USA) statistical software. Considering the slopes of the curves, an inverse function might also work well. Therefore, we also carried out the calculations for $f(n)=a+b / n$ and $f(n)=a+b /(n+c)$. Based on a smaller average $R-s q u a r e$ of the fitted inverse functions, we preferred the exponential model. 


\section{Precision of the measurement}

Two aspects of the precision of a method of measurement can be distinguished: its accuracy and its reproducibility. In this study, reproducibility was most important, because we primarily focused on the differences of a parameter over time in a single individual. To estimate the reproducibility, we needed parameters that were not affected by the possible effects of repeated impacts.

To meet this requirement in testing the reproducibility of the method (the measurements, the correction, and integration process), we reasoned as follows. The velocity and the kinetic energy of the pendulum at impact ( $v_{\max }$ and $E_{k i n}$ ) should be constant throughout a series of impacts. On one hand, the $v_{\max }$ and $E_{\text {kin }}$ can be calculated by means of basic physics from the starting conditions. On the other hand, $v_{\max }$ was found after the first integration of the original signal, $E_{k i n}$ after the second integration from the load-compression curve. Therefore we defined the mean standard deviation (calculated as the square root of the mean of the variances of all the subjects) of $V_{\max }$ and $E_{\text {kin }}$ as a standard of reproducibility of respectively the first and the second integration. These were $0.005 \mathrm{~m} / \mathrm{s}, 0.006 \mathrm{~m} / \mathrm{s}$, and $0.006 \mathrm{~m} / \mathrm{s}$ (respectively $2.3,1.5$ and $0.9 \%$ ) for the first integration and $0.005 \mathrm{~J}, 0.015 \mathrm{~J}$ and $0.022 \mathrm{~J}$ (respectively $4.3,3.3$ and $2.1 \%$ ) for the second integration at respectively $0.2,0.4$ and $0.6 \mathrm{~m} / \mathrm{s}$ impact velocity.

To test the reproducibility of the parameters we determined the interclass correlation coefficient (ICC) of each parameter at each velocity separately, with the random effect model. As the effect of repeated impacts has already died out after the first few impacts ${ }^{5,6}$, we only included the last five impacts of a series of measurements. The ICC of $F_{\text {max }}$ were $0.999,0.999$ and 0.998 , of $E_{\text {dis }} 0.548,0.840$ and 0.955 , of Stiffness $0.991,0.995$ and 0.995 , and of $D_{\max } 0.808,0.950$ and 0.929 at $0.2,0.4$ and $0.6 \mathrm{~m} / \mathrm{s}$, respectively.

\section{Results}

The descriptive statistics of each parameter at $0.2,0.4$ and $0.6 \mathrm{~m} / \mathrm{s}$ show large inter-individual differences (Table 1). With higher impact velocity, the mean values of $F_{\text {max }}$ $D_{\max }$, Stiffness, and $E_{\text {dis }}$ increase. Figure 3 illustrates the results of the relative changes of the values of the parameters on the repeated impacts (and the corresponding p-values). Most of the observed changes take place within the first two to five successive impacts. Subsequent impacts contribute much less to the effect. Figure 4 illustrates the relationship between these changes among the parameters.

The repeated ANOVA-test shows a relation between the impact velocity and the changes of $F_{\max }(p=0.002)$ with a decreasing trend across the impact velocities $(p=0.003)$. For Stiffness the impact velocity was marginally significant $(p=0.071)$, with a decreasing trend $(p=0.057)$. The $D_{\max ,}$ and $E_{\text {dis }}$ showed no significant relation $(p=0.165$ and 0.331 , respectively) (see Fig. 3). 


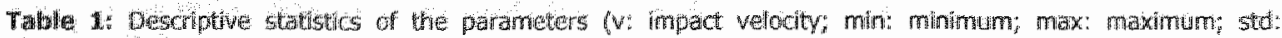
standard deviation).

\begin{tabular}{|c|c|c|c|c|c|}
\hline & $\mathrm{v}[\mathrm{m} / \mathrm{s}]$ & mean & $\min$ & $\max$ & std \\
\hline \multirow{3}{*}{$F_{\text {mats }}[N]$} & 0.2 & 48.2 & 35.4 & 56.3 & 6.6 \\
\hline & 0.4 & 147,6 & 102.2 & 177,3 & 20.0 \\
\hline & 0,6 & 2750 & 177.9 & 340.3 & 39.4 \\
\hline \multirow{3}{*}{$E_{d i s}[J / j]$} & 0.2 & 50.2 & 39.6 & 60.9 & 6.6 \\
\hline & 0.4 & 67.5 & 56.3 & 77.6 & 7.3 \\
\hline & 0.6 & 79.8 & 64.6 & 91.6 & 8.5 \\
\hline \multirow{3}{*}{$\begin{array}{l}\text { Stiffness } \\
{[\mathrm{kN} / \mathrm{m}]}\end{array}$} & 0.2 & 13.3 & 9.1 & 19.4 & 3.9 \\
\hline & 0.4 & 39.0 & 22.4 & 61.3 & 13.9 \\
\hline & 0.6 & 65.7 & 42.0 & 102.5 & 20.3 \\
\hline \multirow{3}{*}{$\mathrm{D}_{\mathrm{m}}$ [mom] } & 0.2 & 7.3 & 6.4 & 8.3 & 0.5 \\
\hline & 0.4 & 9.6 & 8.1 & 11.7 & 1.1 \\
\hline & 0.6 & 11.1 & 9.1 & 13.5 & 1.5 \\
\hline
\end{tabular}

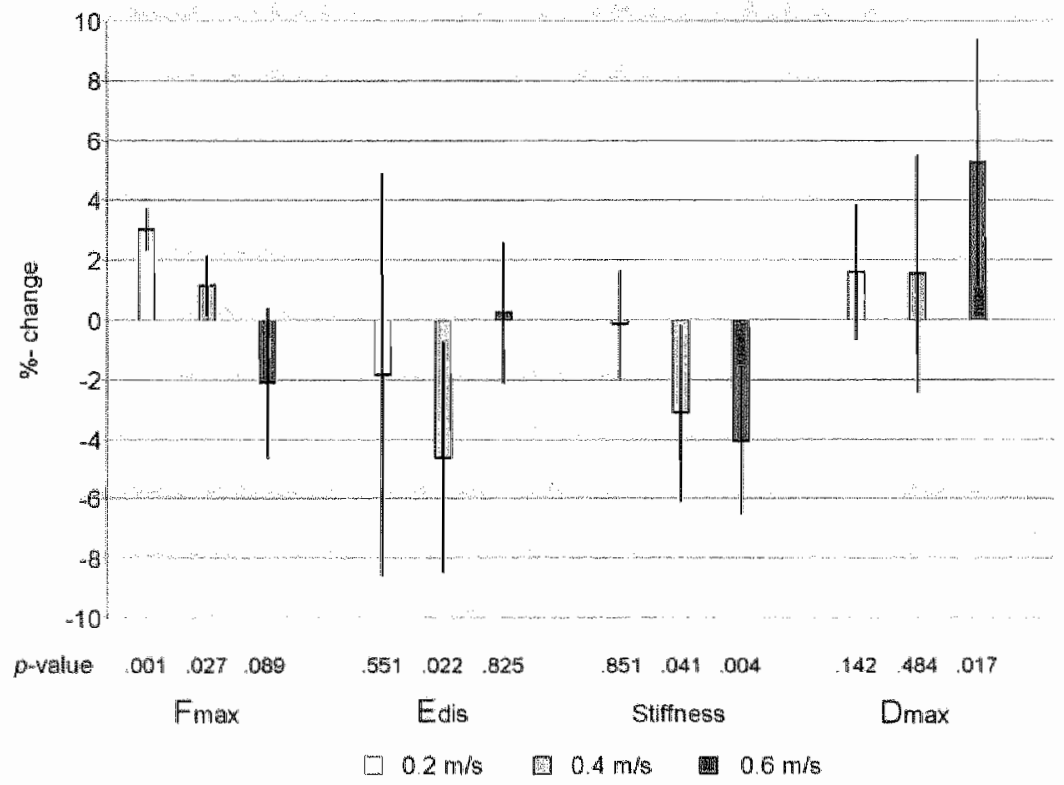

Fig. 3: The mean differences, the $95 \%$-confidence intenal and the p-value of all the parameters at the three impact velocites. The y-axis (\%-change) gives the difference between the first and the eighteenth value of the fitted curve in terms of a percentage. The pwalues denote the of change of a parameter in each volunteer at the same velocity. 


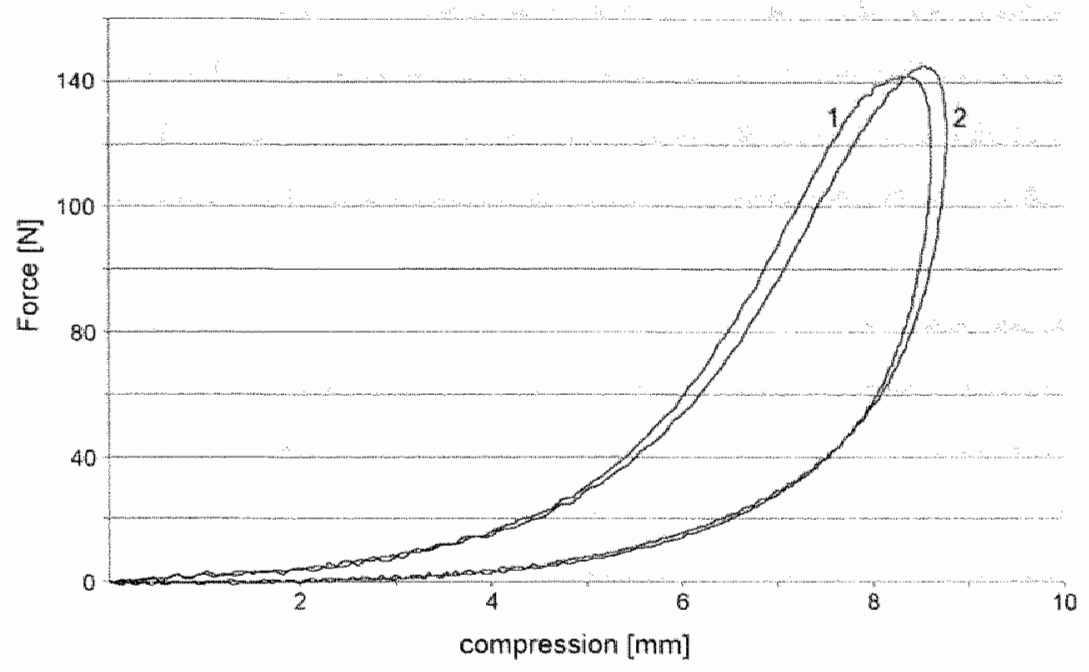

Fig. 4: An example of first (1) and last (2) load-deformation curves of a volunteer at an inpact welocity af 0 . m/s. Note increases in $F_{\text {max }}$ and $D_{\text {max }}$ and decreases in Stiffness and Ess with repeated impacts.

The results on $F_{\max }$ deserved special attention. The effect of repeated impacts on $F_{\max }$ significantly decreases across the velocities from $3.0 \%, 1.2 \%$ to $-2.1 \%$, at $0.2 \mathrm{~m} / \mathrm{s}, 0.4 \mathrm{~m} / \mathrm{s}$ and $0.6 \mathrm{~m} / \mathrm{s}$ respectively. As mentioned above, this trend was significant ( $\mathrm{p}: 0.003$ ). Inspecting the individual curves, at $0.2 \mathrm{~m} / \mathrm{s} F_{\max }$ increased in all subjects (Fig. 5A). At $0.4 \mathrm{~m} / \mathrm{s}_{\mathrm{r}}$ six cases showed the expected shape, which was similar to the curves at $0.2 \mathrm{~m} / \mathrm{s}_{\text {, }}$ but in three cases, at the second impact $F_{\text {max }}$ increased as expected and than started to fall back to the initial value at consecutive impacts (Fig. 5B). At $0.6 \mathrm{~m} / \mathrm{s}$, in six out of eleven cases, $F_{\text {max }}$ increased between the first and the second impact, but then started to decrease even beyond the initial value. In four out of eleven cases, $F_{\text {max }}$ already decreased at the second impact (Fig. 5C). In the cases that were not mentioned, the pattern was undetermined. The inspection of the individual curves of Stiffness at 0.4 and $0.6 \mathrm{~m} / \mathrm{s}$ only showed a gradual fall, without an initiall rise.

\section{Discussion}

The results of this study on one of the fundamental properties of the heel pad confirmed the hypothesized decrease of the $E_{\text {dis }}$ after repeated impacts, but the effect was smal. . This decrease was associated with an increase in $F_{\text {max }}$ inferring a decrease in damping power. To the best of our knowledge, so far only Cavanagh et al. (1984) and Kinoshita at al. (1993) used in wivo impact testing to address the question at hand. In contrast to our results, both authors concluded that there was no effect. Differences in methodology could explain this. Kinoshita et al. discarded 'several practice impact trials' before starting to measure a series of trials. Moreover, they averaged the initial five trials that were included. We found that the effect was largely completed within the first trials. Finally, both Cavanagh et al. and Kinoshita et al. used an impact velocity of about $1.0 \mathrm{~m} / \mathrm{s}$. Most probably, at high impact velocities the effects are too small in relation to the level of energy input. 

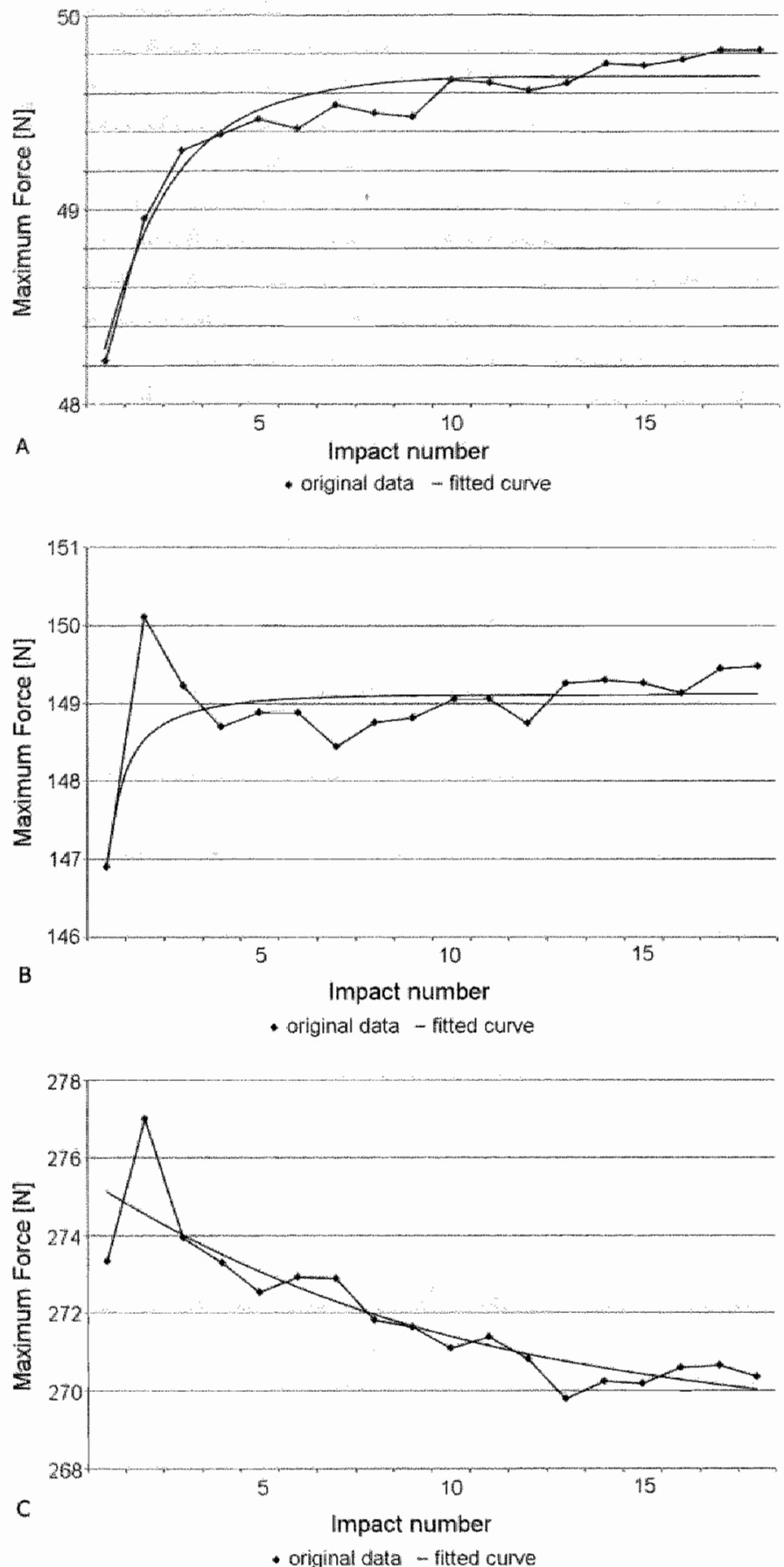

Hig. Th: Thanges of the Fux on repeated impacts of the pendusm at an mpact velocity of $0.2 \mathrm{~m} / \mathrm{s}(\mathrm{A})$, $0.4 \mathrm{~m} / \mathrm{s}(\mathrm{B})$ and $0.6 \mathrm{~m} / \mathrm{s}(\mathrm{C})$. The $\mathrm{n}^{\text {th }}$ polnt of a curve represented the mean watue of the $\mathrm{n}^{\text {th }}$ irmpact in the sequence across all volunteers. Fitted curves are added to illustrate the process of fitung: the actual fitting vas execkited on the curves of each volunteap separately. 
Previously, in vitro experiments with isolated heel pads also reported on the effect of repeated loadings ${ }^{5.6}$. Aerts et al. reported that energy dissipation decreased from $48 \%$ at the first impact to $31 \%$ after many impacts at an "impact" velocity comparable to $0.4 \mathrm{~m} / \mathrm{s}$. Ker reported a decrease from $42 \%$ to $36 \%$, at an impact welocity of $0.15 \mathrm{~m} / \mathrm{s}$ and a rest-time of $20 \mathrm{sec}$. In both investigations, the stiffness increased on repeated impacts. We also found a decrease of the dissipated energy from $68 \%$ to $66 \%$ and from $80 \%$ to $76 \%$ at $0.2 \mathrm{~m} / \mathrm{s}$ and $0.4 \mathrm{~m} / \mathrm{s}$, respectively. The difference in the magnitude of the energy dissipation is a well known phenomenon, that is attributed to the presence of the lower leg "Our decrease in $E_{\text {dis }}$ on repeated impacts was in line with their results, but smaller. The associated decrease in Stiffness was in contradiction with the increase they reported. We are not aware of a biomechanical model of the heel pad that gives a decisive answer to this matter.

At $0.2 \mathrm{~m} / \mathrm{s}_{\mathrm{f}}$ the gradual increase of $F_{\max }$ on consecutive loadings stops after the first impacts. This is in line with the in vitro experiments ${ }^{5}$ and the concept of the visco elastic properties of the heel pad. However, the results of the $F_{\text {max }}$ across the impact velocity show a significantly decreasing trend with even a decrease of the $F_{\max }$ at repeated impacts at $0.6 \mathrm{~m} / \mathrm{s}$ (Fig. 3) that seems to contradict the concept of the visco-elastic properties. Closer inspection of the individual curves at $0.6 \mathrm{~m} / \mathrm{s}$ indeed shows the expected initial increase at the second impact. However, in fitting the exponential function to the data, a decrease of $F_{\max }$ at consecutive impacts overshadowed this initial increase (Fig. $5 \mathrm{C}$ ). This phenomenon is also observed in a few volunteers at $0.4 \mathrm{~m} / \mathrm{s}$ (Fig. 5B). Therefore, we concluded that at all impact velocities the heel pad initially lost the damping power on repeated impacts. The magnitude of this loss was about $3 \%$.

The gradual fall of $F_{\text {max }}$ at 0.4 and $0.6 \mathrm{~m} / \mathrm{s}$ and the contradictory results of stiffness cannot be explained from the material properties of the heel pad only. Therefore, we took a closer look at the possibility of the additional effect of the lower leg. Pain and Challis (2001) constructed a mathematical model to analyse the effect the soft tissue of the shank as a wobbling mass that could move relative to the bones. They concluded that the soft tissue not only dissipated additional energy but also lowered the stiffness of the 'lower leg-system'. This may explain the differences in magnitude of $E_{\text {dis }}$ and Stiffness between the in-vivo and in-vitro experiments, but, as the mass of the lower leg did not change due to repeated impacts, it still could not explain the gradual fall in $F_{\max }$ and stiffness with increasing impact velocity.

Much of the soft tissue mass of the lower leg is made of muscle that might play an active role in the damping of an impact. The heelstrike of locomotion is typically short of duration $(5-20 \mathrm{~Hz})$ and initiates a shockwave that propagates up throughout the body ${ }^{\mathrm{g}-11}$. In the soft tissue, the shockwave induces vibrations. The soft tissues can dampen these vibrations efficiently. The vibration characteristics were changed by muscle activity suggesting an additional strategy to actively adapt to a shockwave ${ }^{12}$. Muscle activity allso couples the soft tissue and the bone to a variable degree. The level of muscle activity in the leg adjusted the loading rate of the impact forces ${ }^{13}$. Remarkably, the change in muscle activity occurred both before and after the impact. These investigations suggest that muscles actively can tune to the repeated impact forces ${ }^{14}$.

Thus, 'tuning" could play a role in the protection of the musculo-skeletal system from detrimental impact forces. In this context, our results support these alternative strategies to cope with impact forces. At an impact velocity of $0.2 \mathrm{~m} / \mathrm{s}$, the effect of repeated impacts on the parameters was in line with the visco-elasticity and the impact force did not provoke active tuning. At 0.4 and $0.6 \mathrm{~m} / \mathrm{s}$ in some volunteers, visco-elasticity cannot explain the effect and active tuning likely occurred. Note that in our study $0.6 \mathrm{~m} / \mathrm{s}$ was the upper limit of tolerance and it would be natural to provoke a reaction. In addition, the gradual decrease on consecutive impacts in some subject rather points to fine-tuning than to a material property. 


\section{Limitations}

In the pendulum impact tests, the direction of the reaction force is ideally directed along the axis of the bar of the pendulum. In the actual situation, the impact region on the heel pad is not everywhere parallel to the front face of the bar. Moreover, the internal structure of the heel paid and the shape of the underlying calcaneal bone are not identical at every spot. Thus, in the real experiments the reaction force might not always be fully aligned with the bar's axis, as previously was observed should the non-axial force arise, it could give axial rotation and a lateral swing to the bar and as a consequence unaccountable loss of energy. Based on the physics of inertia and a valuation of the lateral deflection (amplitude) at $1 \mathrm{~cm}$, we estimated that the loss in such cases would be less than $2 \%$ of the kinetic energy input of the pendulum.

We incidentally observed a lateral swinging movement of the pendulum after impact. Nevertheless, we included these impacts. As the energy is lost in the out-going phase, the reproducibility of the $E_{\text {dis }}$ would be affected most. This is in line with the results of the ICC.

We put quite an effort in stabilizing the lower leg and ankle. After a series of measurement, we verified the match in position of the pendulum in equilibrium and the mark on the skin. However, this did not exclude movement during a single impact. Especially, this went for the movement in the axilal direction. We had no means to verify this. As this movement can be considered as an unavoidable systematic error, we do not think that it affected the results, other than increasing their standard deviation.

Between the series of consecutive impacts, the preparation time was about two minutes only. This rest-time may not have been sufficient for the heel pad to fully relax. If so, the observed decrease in clamping and energy dissipation are underestimated.

\section{Clinical context}

The heelstrike transient or impact force is a short spike of force superimposed on the flatter upslope of the ground reaction force, that follows immediately after initial contact of the heel with the ground ${ }^{15}$. Because this impact force characteristically is shorter of duration (in running $10-20 \mathrm{~ms}$ ) than the latency time of the skeletal muscle ${ }^{15}$, the impact force is only passively damped. However, the above is a simplification of the real situation. The repetitive nature of locomation, gives the musculaskeletal system the opportunity to anticipate on the expected next impact to minimize the associated "shockwave". This consideration leaves the observation unimpeded, that in the skeleton the impact force causes a 'shockwave' that propagates upward, and even reaches the skull ${ }^{\$, 10,17}$. Clinically, this was supposed to be important because improper attenuation of the impact force was said to be an etiological factor in the development of conditions such as degenerative joint disease and low back pain ${ }^{11}$. More recent publications contradict this ${ }^{18}$. As this discussion is not concluded, our abserved decrease in damping power on repetitive impacts is potentially detrimental. However, pendulum impact studies merely are one-dimensional investigations of the three dimensional phenomenon of the heelstrike. Thus, our results are a simplification of reality. In reality, every step differs slightly from the previous or the next one. At heelstrike, the contact area will be slightly different at every step. Moreover, during the stance phase the heel is deformed in a different way. It is therefore likely that part of the loss of the damping power is preserved at repetitive activities with a low impact velocity, such as walking. The previous remarks do not make our observations meaningless. Shoes tend to limit the ability for the heel to move around and avoid repetitive impacts on the same spot. Therefore, is seems prudent to wear shoes that provide sufficlent space. Moreover, even in walking, the peak transient deceleration of the tibia at heell strike reached approximately $2-7 g^{11_{*} 19}$. Minor loss of damping of the impact force may give considerable effect certainly when the repetitive character of locomotion is considered. 


\section{Conclusion}

Based on the visco-elastic properties of the heel pad we hypothesize a decrease in energy dissipation of the heel pad on repeated impacts. Our resuits confirm the hypothesis and show that the decrease in energy dissipation that is associated with a decrease in the maximum force and stiffness on consecutive impacts. However, visco-elasticity cannot explain the relation of increasing impact velocity and decreasing maximum force and stiffness. The relation suggests an additional damping action of the muscles of the lower leg. Most likely, this is due to tuning of the muscles to repeated impacts, in order to decrease the impact force. If this assumption is correct, this damping mechanism of the lower leg more than compensates the loss in damping power of the heel pad. Although our observations on the lower leg in relation to the heel pad primarily are of theoretical interest, they may improve insight in the physiology of locomotion.

\section{Acknowledgements}

We thank Martin van der Wolf and Emiel Arnoldussen of the IDEE of the University Maastricht for their technical support and the workers of the orthopaedic outpatient ward for their assistance.

\section{References}

1. Jorgensen $U$ and Bojsen-Moller $F$ : Shock absorbency of factors in the shoe/heel interaction with special focus on role of the heel pad. Foot Ankle 1989; 9: 294-299.

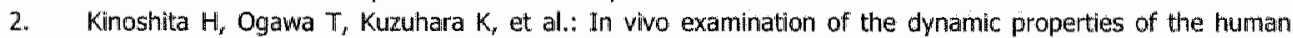
heel pad. Int I Sports Med 1993; 14: 3:12-319.

3. Miller-Young JE, Duncan NA and Baroud G: Material properties of the human calcaneal fat pad in compression: experiment and theory. J Biomech 2002; 35: 1523-1531.

4. Sarrafian SK: Functional anatomy of the foot and ankle. In Anatomy of the Foot and Ankle, edited by Sarrafian SK. second edition. Philadelphia; Lippincot J.B. 1993. 474-602.

5. Ker RF: The time-dependent mechanical properties of the human heel pad in the context of locomotion. J Exp Biol 1996; 199: 1501-1508.

6. Aerts P, Ker RF, De Clercq D, et al.: The mechanical properties of the human heel pad: a paradox resolved. J Biomech 1995; 28: 1299-1308.

7. Cavanagh PR, Valiant GA and Misevich KW: Biological aspects of modeling shoe/ foot interaction during running. In Sport shoes and playing surfaces, edited by Frederick CA. Champaign, Illinois; Human Kinetics Publishers, Inc. 1984, 24-46.

8. Weijers RE, Kessels, A.G.H. Kemerink, G.J.: The damping properties of the venous plexus of the hael region of the foot during simulated heel strike. J Biomech in press;

9. Voloshin AS: Shock absorption during running and walking. J Am Podiatr Med Assoc 1988; 78 : 295-299,

10. Wosk J and Voloshin A: Wave attenuation in skeletons of young healthy persons. J Biomech 1981; 14: $261-267$.

11. Collins $\mathrm{JJ}$ and Whittle MW: Impusive forces during walking and their clinical implications. Clin Biomecth 1989; 4: 179-187.

12. Wakeling JM and Nigg BM: Modification of soft tissue vibrations in the leg by muscular activity. J Appl Physiol 2001; 90: 412-420.

13. Wakeling JM, Von Tscharner $V$, Nigg BM, et al.: Muscle activity in the leg is tuned in respanse to ground reaction forces. J Appl Physiol 2001; 91: 1307-1317.

14. Boyer KA and Nigg BM: Muscle activity in the leg is tuned in response to impact force characteristics. 3 Biomech 2004; 37: 1583-1588. 
15. Whitue MW: Generation and attenuation of transient impulsive forces beneath the foot: a rewiew. Gat Posture 1999; 10: $264 \times 275$.

16. Nigg BM, Denoth , Kerr B; et al: Load sport shoes and playing surfaces. In Sport shoes and playing surfaces, edited by Frederick CA. Champaing, Illinois; Human Knetics Publishers, InC. 1984, 1-23.

17. Vallant GA Transmission and attenuation of heelstrike accelarations. In Biomechanics of distamce runming, edited by Cavanagh PR. Champagn, Illinois, fimman Kinetics Publishers, InC. 1990. 225-247.

18. Nigg BM and Wakeling JM, Impact forces and muscle tuning: a new paradigm. Exerc Sport So Rev 2001; 29: 37.41 .

19. Maclellan GE; Skeletal heel strike transelents, measurement; implications, and modif cation by footwear. In Sport shoes and playing surfaces, edted by Frederick CA. Champaign, Illinois; Human lKinetics Publishers, Inc $1984,76-86$. 


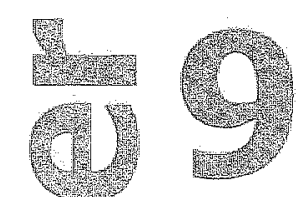

The damping properties of the venous plexus of the heel region of the foot during simulated heelstrike

RE Weijers, AGH Kessels, and GJ Kemerink

Journal of Biomechanics, in press

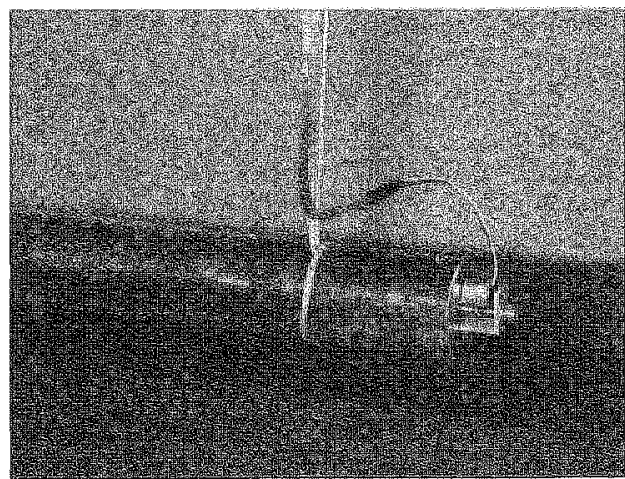




\section{Abstract}

The damping mechanisms that are operational in the heel pad during the impact phase of locomotion have the important function to protect the musculo-skeletal system from njuries. How this is achieved is still not fully understood, as is for instance illustrated by the heel pad paradox, the observation that in in-vivo and in-vitro experiments yielded widely different results. This paradox could so far only partially be explained. In the light of this paradox, and a previous study by our group, we conjectured that the venous plexus might contribute as a hydraulic shock absorber to the damping properties of the heel pad To investigate this hypothesis in wivo, heel pads of 11 volunteers were subjected to pendulum impact tests, using velocities of $0.2,0.4$, and $0.6 \mathrm{~m} / \mathrm{s}$, and three physiologically different, consecutive conditions: (0) a relatively empty venous plexus, (ii) a congested venous plexus, and (ii) a decongested venous plexus. At congestion, the maximum impact force decreased slightly but significantly by $2.6 \%$ at $0.2 \mathrm{~m} / \mathrm{s}$ and $1.8 \%$ at $0.4 \mathrm{~m} / \mathrm{s}$. This effect was no longer found at $0,6 \mathrm{~m} / \mathrm{s}$. Although these effects are rather small, they confirm the fundamental hypothesis that the venous plexus contributes to the damping properties of the heel pad during walking. It is likely that some underestimation of the effect has occurred. 


\section{Introduction}

Neutralization of the effects of the impact forces that arise during heel strike in locomotion is essential in preventing damage to the musculo-skeletal system " Notwithstanding several studies, the mechanisms that damp the impact are still not fully understood. Based on the results of a prevlous study ${ }^{2}$, we hypothesised that the venous structures in the soft tissue of the heel pad might contribute to the damping by functioning as a hydro-mechanical shock absorber. In a search in the available literature on the mechanical properties of the heel pad no systematic study on this matter was found. We did find controversial results in the reported mechanical properties of the heel pad, however. Most striking were the differences between studies conducted on healthy volunteers and studies conducted on isolated cadaver heel pads, "the heel pad paradox" ${ }^{3}$. Although methodological factors explained most of these differences, a functional venous plexus in the in vivo test might also explain part of the 'paradox'. The reported haemolysis in long distance runners corroborates a relation between mechanical loading and the presence of blood in the sole of the foot ${ }^{4}: 5$. With this in mind, we conducted this study to quantitatively answer the question whether the venous plexus influenced the mechanical properties of the heel pad. The results would be of fundamental interest in basic science, and might also improve the insight in the patho-physiology linked to impact forces during locomotion.

\section{Material and methods}

\section{Subjects}

The subjects were healthy males, without physical complaints of their feet. No plantar callus formation was present. Eleven subjects were included (mean age: 38.1 years, range: 29-54; mean weight: $79.1 \mathrm{~kg}$, range: $70-89$; mean length" $1.82 \mathrm{~m}$; range: $1.74-1.93$ ). The study was approved by our Medical Ethic Committee. All volunteers gave informed consent.

\section{Principle of the experiments}

The effect of the venous plexus was investigated by comparing pendulum impact experiments on heel pads with empty veins and heel pads with blood-filled veins. During the experiment, the volunteers lay supine on the floor with their lower leg above the level of the body. In this position, the venous bload pressure approaches $0 \mathrm{~mm} \mathrm{Hg}$, instead of about: $80 \mathrm{~mm} \mathrm{Hg}$ in an erect person, standing perfectly still. Thus, a relatively empty venous plexus was obtained. Filling of the venous plexus of the calf was realised by restriction of the venous outflow with a sphygmomanometer, inflated with a pressure of $80 \mathrm{mmHg}$.

\section{Position of the volunteers}

Before positioning, the volunteers uncovered their left lower leg to avoid unintentional venous congestion. A cuff of a sphygmomanometer was wrapped around the lower leg just below the knee. During the experiment, the volunteers lay supine on the ground beside a massive rectangular concrete pillar. The hip was flexed a little over $90^{\circ}$ and slightly abducted; the knee was flexed a little over $90^{\circ}$. Thus, the lateral femoral condyle could be placed against the flat surface of the rectangular concrete pillar with the lower leg in horizontal position. To secure this position, the leg was placed in a homemade 'vices. The jaws of the vice compressed the lower leg over the medial and lateral side of the tibia plateau and the malleoli (Fig. 1A). Moreover, it supported the dorsal part of the heel in such a way that the calf was free from any contact with the vice to avoid unwanted venous congestion. To reduce the damping effect by the soft tissue between the lateral femoral condyle of the knee and the concrete wall, the lower leg was pulled against the pillar with 
about 70 N. For this purpose we used a removable plaster cast that served as a 'stirrup' and a spring-based weighing device to adjust the force. A strap connected the cast to the weighing dewice that was fixed to the pillar (Fig. 1B). The cast covered the foot and the distal lower leg with the exception of the plartar side of the heel.

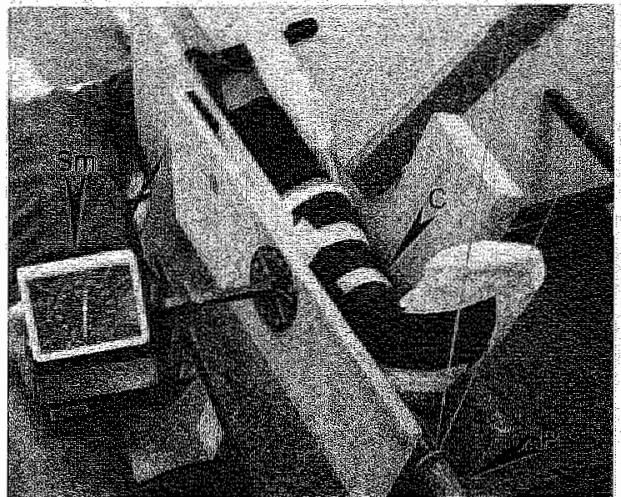

A

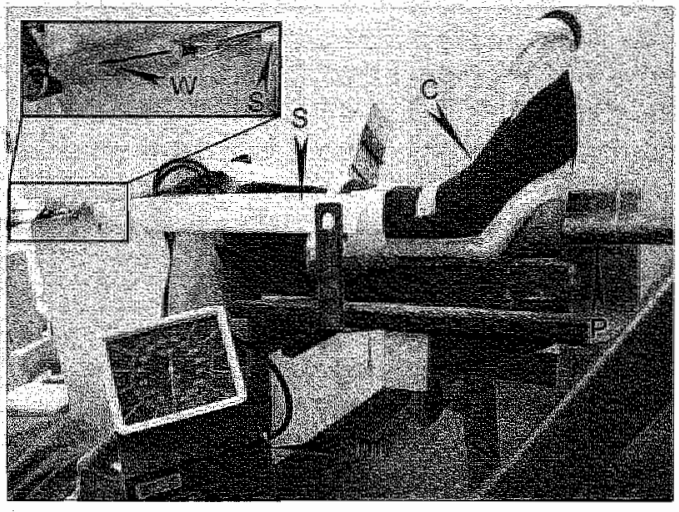

.

Fig. 1: A view from above (A) and a view side long (B) of the position of the wolunteer and the pendulum during the experiment. For the sake of darity we removed the "jaw" of the vice in (B). (C: cast; p: pendulum; 5 : strap; sm= sphygromanometer: W: whenging device).

\section{Pendulum}

The pendulum consisted of a cylindrical steel bar suspended by cords in a frame on wheels. The bar had a length of $67.0 \mathrm{~cm}$, a cross section of $9.62 \mathrm{~cm}^{2}$ and a mass of $5.1175 \mathrm{~kg}$. The effective length of the suspending cords was $1.5025 \mathrm{~m}$, its free swing period $2.46 \mathrm{~s}$. From each end of the bar, two cords were connected to points on the frame that were $30 \mathrm{~cm}$ apart in the lateral direction. By using these four cords, instead of simply two, transwersal oscillations could largely be suppressed. The steel bar was instrumented with a band-pass mono-axial accelerometer (model PB3ACW, Oceana Sensor, Virginia Beach, VA) with the frequency response within $\pm 5 \%$ from $1 \mathrm{~Hz}$ to $4 \mathrm{kHz}$. The signal of the accelerometer was digitised using a multifunction PC board (AT-MIO-16E-10, National Instruments, Austin, TX). This board had an adjustable gain amplifier and a 12-bit analog to digital converter.

The accelerometer's sensitivity and low-off time-constant were calibrated in two different ways. (i) The calculated acceleration from a freely oscillating pendulum was compared with measured values. (ii) The pendulum was allowed to impact on a thick felt layer, while the initial amplitude and amplitude after impact were measured. Using the equation of the pendulum, the expected accelerations were calculated and compared with the measured results. The former method tested the behaviour at small accelerations; the second method was more representative for the actual measurements. These calibrations resulted in a sensitivity of $99.0 \mathrm{mV} / \mathrm{g}$, with $\mathrm{g}$ the gravitational constant and a time constant (RC) of $0.53 \mathrm{~s}$ for the low frequency roll-off. Both values were close to the nominal values specified by the manufacturer $(99 \mathrm{mV} / \mathrm{g}, 0.5 \mathrm{~s})$. The calibration experiments also showed that sample rates between $1 \mathrm{kHz}$ and $10 \mathrm{kHz}$ gave identical results for all parameters later used in the clinical experiments. To be on the safe side, $5 \mathrm{kHz}$ was used in all experiments. The duration of the sampling period was 5 seconds, including $1 \mathrm{~s}$ for measurement of the baseline signal.

\section{The measurements}

Prior to the experiment, the skin over the tuber calcanei was marked under ultrasound guidance. The mark served as the centre of the impact region. After the pendulum was aligned with the axis of the lower leg, its bearing structure was clamped between two walls 
to prevent ascillations of the frame. In equilibrium, the centre of the end of the bar just touched the marked skin of the heel through the defect in the plaster (Fig. 1), The velocity $(0.2,0.4$ and $0.6 \mathrm{~m} / \mathrm{s})$ of the impacting bar was chosen by the out of equilibrium amplitude of the bar at the moment it was released. At each velocity we measured the impacts of the pendulum for six minutes, at a rate of about three impacts a minute. Such a sequence (18 trials in total) was divided into three stages. In Stage 1 , three baseline trals were made. Next, the cuff around the lower leg was inflated to $80 \mathrm{mmHg}$, which should be ample to induce venous congestion in all healthy volunteers. For a period of $2.5 \mathrm{~min}$ the cuff remained inflated (Stage 2,8 trials). Finally, the cuff was deflated and we continued measuring for another $2.5 \mathrm{~min}$ (Stage 3,7 trials).

Because repeated impacts of the pendulum on exactly the same spot of the heel might have an effect of their own, we performed a 'dummy' procedure within two weeks of the 'congested' procedure. This 'dummy' procedure was a copy of the 'congested' procedure except for Stage 2 in which we now did not inflate the cuff.

\section{Data pre-processing}

Data analysis was done in Matlab version 6.1 (The MathWorks Inc, Natick, MA, USA). The only user interaction consisted in specifying the data point where the pendulum had been released.

Data pre-processing started with a correction for the (single pole) response fall-off at low frequencies. This was done in the frequency domain according to

$$
F V_{\text {corr }}(f)=F V_{\text {uncorr }}(f) / H_{\text {singisepole }}(f) \text {, }
$$

with $\mathrm{FV}_{\text {uncorr }}(\mathrm{f})$ the fast Fourier transform (FFT) of the measured signal voltage, at frequency $f$. $F V_{\text {corr }}(f)$ the corrected signal in the frequency domain, and $H_{\text {singlepole }}(f)$ the function describing the frequency response of the transducer at low frequencies:

$$
H_{\text {singlepose }}(f)=\frac{1}{1+\frac{1}{i 2 \pi / R C}},
$$

with RC the time constant ( $0.53 \mathrm{~s})$ describing the fall-off, and $i=\sqrt{ }(-1)$. In this correction the zero frequency component, corresponding to the DC component in the measured voltage, was set to zero (i.e. $F V_{\text {uncorr }}(0)=0$ ), and we set $H_{\text {singlepole }}(0)=1$ to prevent division by zero. After transforming back to the time domain, the DC signal was restored by calculating a correction that resulted in a zero average of the baseline signal and applying this correction to all measured points. Corrections to be applied due to high-frequency fall-off were negligible and were therefore not implemented.

The next steps consisted in calculating the acceleration, the force, the velocity and the position of the pendulum. In the following ' $n$ ' indicates the number of the sample in the acquired time series:

$$
\text { Acceleration }(n)=\left(V_{\text {corr }}(n) / \text { transducer sensitivity }\right)^{*} g_{\text {grawty, }}
$$

with $V_{\text {corr }}$ the corrected transducer signal and $g_{\text {gravity }}=9.82 \mathrm{~m} / \mathrm{s}^{2}$.

$$
\text { Force }(n)=\text { mass_pend * Acceleration( } n \text { ) }
$$

Velocity and position were obtained by integration using the trapezoidal rule:

Velocity $(0)=0$; Velocity $(n)=\operatorname{Velocity}(n-1)+0.5 *($ Acceleration $(n)+$ Acceleration $(n-1))$ * $d_{t}$ with dt the time between two samples during acquisition ( $0.2 \mathrm{~ms})$.

$$
\text { Position }(0)=\text { start_amplitude }
$$

$$
\operatorname{Position}(n)=\operatorname{Position}(n-1)+0.5 * \text { Velocity }(n)+\text { Velocity }(n-1)) * d t
$$

From these results force-position (or load-deformation) curves were constructed (Fig. 2). The energy transferred to the heel was obtained by integration of the force over the path followed during the in-going part of the collision. Similarly, the energy returned to the 
pendulum was calculated over the out-going path. The integration intervals (in-going and out-going) were determined fully automatically. The square root of the force-position curve upon impact is linear over a considerable range. This was exploited by fitting this curve with a line over the range corresponding to $1.5 \%$ to $15 \%$ of the maximum force. The intercept with the baseline (force-position curve just before impact) was taken as the point of impact. Similarly, we calculated the position where contact is lost between pendulum and object. The depth of impact was calculated as the difference in distance between the point of impact and the greatest depth reached by the pendulum.

\section{Definition of the parameters}

From a load-deformation curve (Fig. 2) the following parameters were calculated:

- Finax maximum force ( $=$ mass of pendulum * maximum deceleration) [N]

- Dmax: maximum compression [mm]

- Stiffiness: the coefficient of the linear term in the regression equation fitted to the linear part of the load-compression curve $[\mathrm{kN} / \mathrm{m}]$.

- $E_{\text {dfis: }}$ dissipated energy: the lost energy expressed as a percentage of the kinetic energy of the pendulum at the beginning of the impact. It is defined as (area under the loaddeformation curve during compression minus the area during recoil)*100/ area under the laad-deformation curve during compression.

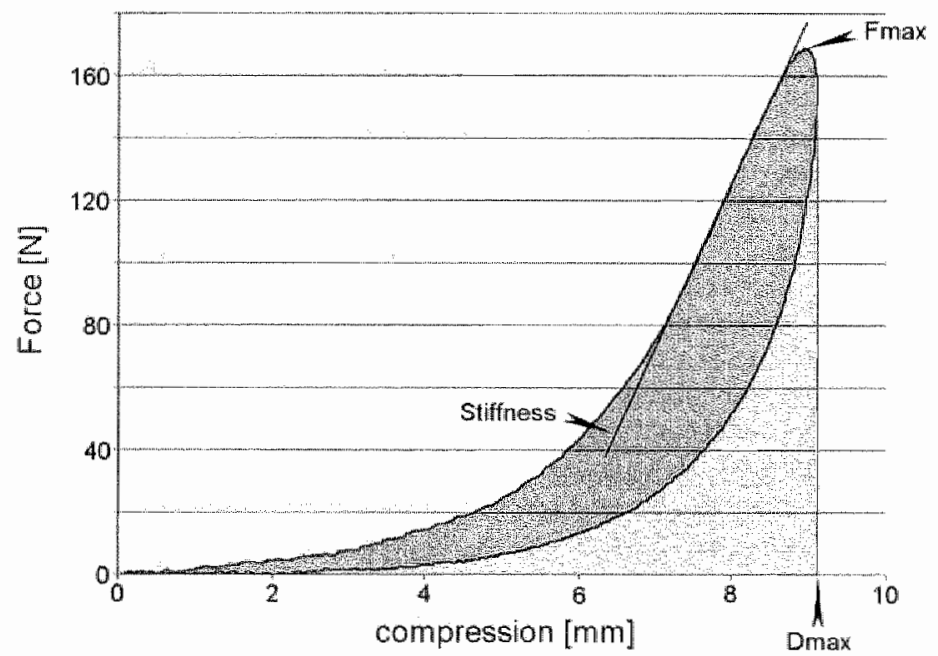

Filg. 2: An axmple of a load-defomation curve of a volunteer at $0.4 \mathrm{~m} / \mathrm{s}$. The Fmax watrectly catculated from

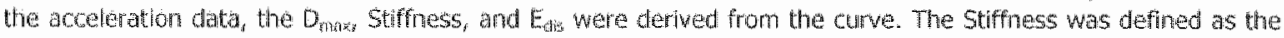
regression coeffictent of a regression line fitted to the linear part of the curve. The E. was defined as: the dark gatey area 100 : llight grey plus dark grey area).

\section{Statistical analysis}

Of each parameter, the averages of the measurements in Stage 1, Stage 2, and Stage 3 of a sequence were calculated (Fig. 3). To find the gross effect of the congestion, the average of Stage 1 was subtracted from Stage 2 . To find the gross effect of decongestion, we subtracted Stage 2 from stage 3. To correct for possible effects of repeated impacts, we treated the data of the dummy procedure the same way. The outcomes of the congested (the gross effect) and the dummy procedure (the correction) of each volunteer were paired (Fig. 4). For each parameter, this algorithm was applied to the data of all volunteers at the three impact velocities. Finally, the results were statistically analysed. 
The null hypothesis, that there were no effects of venous congestion (difference between the means of Stage 1 and 2) or decongestion (difference between the means of Stage 2 and 3), was tested. This was done for all four parameters at the three impact velocities Two tests were used:

- the two-tailed studient t-test on all four parameters at all three impact velocities.

- the ordinary least squares (OLS) test as described by $\mathrm{O}^{\prime} B \mathrm{Brien}$ and modified by Lauter ${ }^{6}$ : In this tests the outcomes of the three impact velocities were pooled for each parameter to increase the power. It gives the overall tendency of the parameter and the $p$-value.

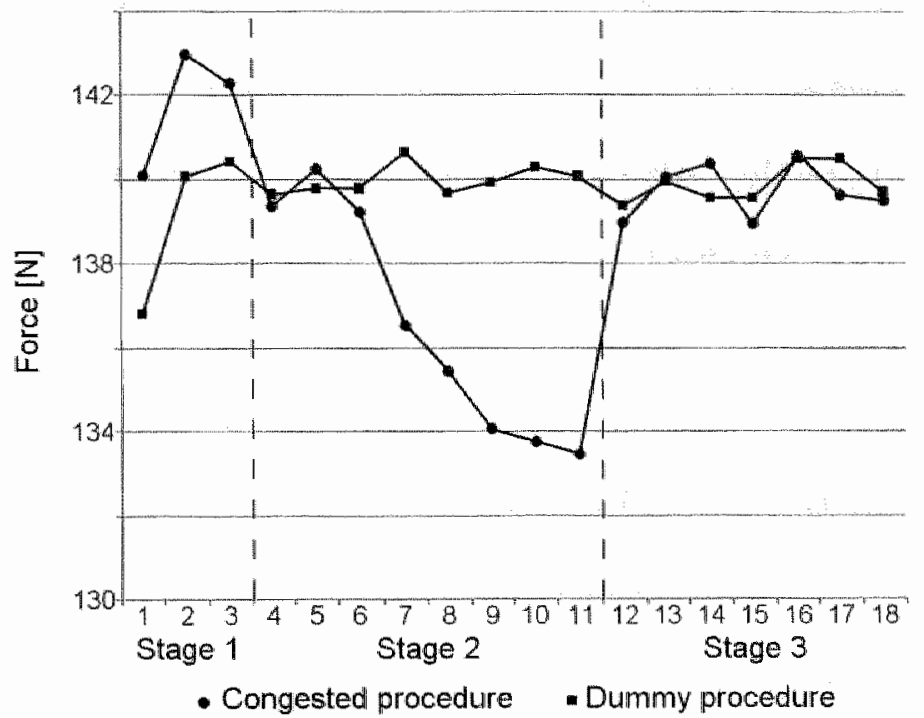

Fig. 3: An example of a complete examination in one stubject. It illustrated the changes over time of the maximum force at an impact velocity of $0.4 \mathrm{~m} / \mathrm{s}$. The $\mathrm{n}^{\mathrm{th}}$ point on the curve rastesented the $\mathrm{n}^{\text {th }}$ impact in the sequence. Only in the 'congested' procedure the ouf was inflated between the $3^{\text {sid }}$ and the $4^{\text {th }}$ impact and deflated between the $1^{\text {th }}$ and $12^{\text {th }}$ impact.

\section{Congested procedure Dummy procedure}

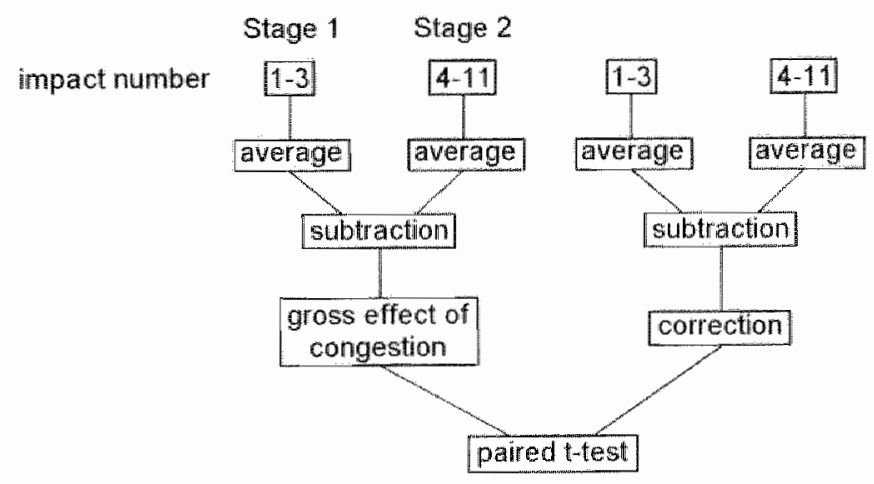

Fig. 4: Sthematic representation of the andysis of the effert of congestion. This algorithm was carried out for each subject and parameter at all the dhree impact velocites. The analysis of decongeston was done in a sirnili way. 


\section{Reliability of the measurement}

Two aspects of the reliability of a method of measurement can be distinguished: its accuracy and its reproducibility. As we primarily focused on the differences of a parameter over time in a single individual, reproducibility was most important. To indicate the reproducibility, we reasoned as follows.

By their origin, the parameters could be split up into two groups. The $F_{\max }$ was directly related to the digitised acceleration signal. The $D_{\text {max }}$ Stiffness and $E_{\text {dis }}$ were calculated from the load-deformation curve that was derived from the original signal by way of a double integration. It was to be expected that the relative error of these parameters would be different from the original signal.

Basic physics describes the relation between the pendulum"s initial position and acceleration due to gravity on the one hand, and the velocity and the kinetic energy of the pendulum at the moment of impact ( $V_{\max }$ and $E_{k m}$ ) on the other. Variations in e.g. the amplitude at the start of a series of impacts could cause differences between individuals. But in a single individual, the $v_{\max }$ and the $\mathrm{E}_{\text {kin }}$ should be constant throughout a series of impacts. The $\mathrm{v}_{\max }$ was found after the first integration of the original signal, the $E_{k i n}$ after the second integration. Therefore, we could define the mean standard deviation (calculated as the square root of the mean of the variances of all the volunteers) of the $v_{\max }$ and the $E_{k i n}$ as a standard of reproducibility of respectively the first and the second integration. These were $0.005,0.006$, and $0.006 \mathrm{~m} / \mathrm{s}$ (respectively $2.3,1.3$ and $0.9 \%$ ) for the first integration and $0.005,0.013$, and $0.021 \mathrm{~J}$ (respectively $4.6,2.9$ and $2.1 \%$ ) for the second integration at, respectively, 0.2, 0.4 and $0.6 \mathrm{~m} / \mathrm{s}$ impact velocity. Quite some effort was spent in optimising the experimental set-up to reach this reproducibility.

\section{Results}

The statistical descriptions of the parameters at the three impact velocities were summarised in Table 1. To test the effect of venous (de)congestion on the parameters at the three impact velocities separately, the two-tailed student $t$-test was used. The results were summarised in Table 2. At congestion, the $F_{\max }$ decreased significantly from $2.6 \%$ at $0.2 \mathrm{~m} / \mathrm{s}$ to $0 \%$ at $0.6 \mathrm{~m} / \mathrm{s}$. Decongestion gave a (smaller) opposite effect, although not significant at all velocities.

Table 1: The deschptwe statisto for 11 subjects of the means in stage 1 (emptr venous piexus), 2 (venots

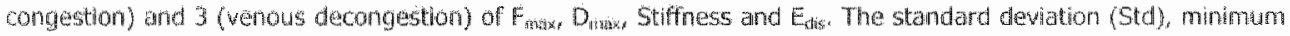
(mn), and maximumax) redated to stage 1.

\begin{tabular}{|c|c|c|c|c|c|c|c|}
\hline & velocity & Stage 1 & Stage 2 & Stage 3 & Std & $\min$ & $\max$ \\
\hline \multirow{3}{*}{$\begin{array}{l}F_{\text {max }} \\
{[N]}\end{array}$} & 0.2 & 50 & 49 & 50 & 8 & 39 & 67 \\
\hline & 0.4 & 149 & 147 & 1.48 & 25 & 103 & 186 \\
\hline & 0.6 & 271 & 268 & 268 & 53 & 172 & 364 \\
\hline \multirow{3}{*}{ 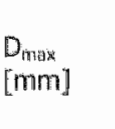 } & 0.2 & 7.7 & 7.6 & 7.7 & 0.8 & 6.4 & 8.5 \\
\hline & 0.4 & 9.7 & 9.8 & 9.8 & 1.1 & 7.3 & 10.8 \\
\hline & 0.6 & 11.1 & 11.5 & 11.3 & 1.5 & 7.9 & 12.6 \\
\hline \multirow{3}{*}{$\begin{array}{l}\text { Stiffness } \\
\text { [kN/mim] }\end{array}$} & 0.2 & 13.7 & 13.5 & 13.7 & 4.0 & 10.0 & 22.3 \\
\hline & 0.4 & 39.3 & 37.3 & 38.0 & 14.7 & 25.0 & 68.3 \\
\hline & 0.6 & 65.7 & 62.6 & 62.7 & 27.2 & 33.8 & 119.4 \\
\hline \multirow{3}{*}{$E_{d i s}$} & 0.2 & 53 & 51 & 52 & 6 & 46 & 63 \\
\hline & 0.4 & 65 & 63 & 64 & 4 & 59 & 71 \\
\hline & 0.6 & 77 & 77 & 78 & 4 & 72 & 85 \\
\hline
\end{tabular}


The Stiffness decreased on congestion, and increased on decongestion. Only the data at: $0.4 \mathrm{~m} / \mathrm{s}$ were significant. The effect probably will be about $3 \%$, but a velocity dependency cannot be concluded from these data. The results of $D_{\text {niax }}$ and Edis were not statistically significant.

The overall results of the OLS-test (Table 3) showed a decrease in the $F_{\text {max }}$ and the Stiffness at congestion $(p=0.003$ and $p=0.068)$ and a tendency towards the opposite effect at: decongestion $(p=0.096$ and $p=0.065)$. On the $E_{d i s}$ and the $D_{n \text { max, }}$ no effect of venous congestion was found.

Table $2:$ The effect of wenous congestion (Stage 2 minus Stage 1 ) and deomgestion (Stage 3 minus stage 2 ) or

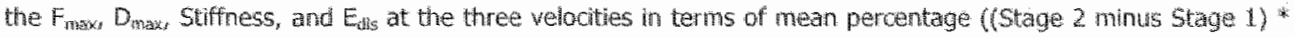
$100 /$ Stage 1). (v: velocity [nv/s]). P-values $<0.05$ are printed in bold.

\begin{tabular}{|c|c|c|c|c|c|}
\hline & & con & & decon & ted \\
\hline & v & $\%$ & $p$ & $\%$ & $p$ \\
\hline & 0.2 & -2.6 & 0.000 & 1.5 & 0.041 \\
\hline $\begin{array}{l}F_{\text {matix }} \\
{[N]}\end{array}$ & 0.4 & -1.8 & 0.003 & 0.7 & 0.207 \\
\hline & 0.6 & 0.2 & 0.972 & -0.7 & 0,525 \\
\hline & 0.2 & -1.7 & 0.229 & 0.2 & 0.885 \\
\hline$D_{\max }$ & 0.4 & 1.2 & 0.295 & -0.4 & 0.635 \\
\hline & 0.6 & 1.8 & 0.394 & -2.7 & 0.033 \\
\hline & 0.2 & -1.3 & 0.316 & 2.3 & 0.179 \\
\hline $\begin{array}{l}\text { Stittness } \\
{[\mathrm{kN} / \mathrm{m}]}\end{array}$ & 0.4 & -3.7 & 0.024 & 2.7 & 0.027 \\
\hline & 0.6 & -1.9 & 0.229 & 1.1 & 0.292 \\
\hline & 0.2 & -1.5 & 0.592 & -0.2 & 0.957 \\
\hline Edis & 0.4 & 1.1 & 0.514 & 0.7 & 0.560 \\
\hline & 0.6 & -0.2 & 0.877 & 0.8 & 0.152 \\
\hline
\end{tabular}

Table $3:$ The results of the oL 5 test. Congestion: Stage 2 minus Stage 1 dedongestion: Stage 3 minus Stage 2). P-values $<0.05$ are printed in bold.

\begin{tabular}{|c|c|c|c|c|}
\hline & \multicolumn{2}{|c|}{ congestion } & \multicolumn{2}{|c|}{ decongestion } \\
\hline & $t$ & $p$ & $\mathrm{t}$ & $p$ \\
\hline$\overline{F_{\text {max }}}$ & -3.8 & 0.003 & 1.8 & 0.096 \\
\hline $\mathrm{D}_{\max }$ & 0.3 & 0.758 & -1.5 & 0.177 \\
\hline Stiffness & -2.0 & 0.068 & 2.1 & 0.065 \\
\hline$\underline{E}_{\text {dis }}$ & -0.01 & 0.992 & -1.9 & 0.081 \\
\hline
\end{tabular}

\section{Discussion}

The results of this study support the fundamental hypothesis that part of the damping properties of the heel pad comes from a distended venous plexus. Nevertheless, its: contribution appears to be limited, even if some underestimation is taken into account.

At the end of the swing phase of gait, the foot contacts the ground and generates a reactive force. This force starts with a short spike with a duration of $10-20 \mathrm{~ms}$ that is superimposed on the slower upslope of the ground reactive force. This high-frequency force travels up the skeleton and is called the impact force or heelstrike transient 7,8 . There is evidence that this force is linked to many pathological conditions, such as low back pain and osteoarthritis, as reviewed by collins " Damping the impact force might reduce the incidence of these 
conditions. The human heel pad has been shown to be the most important damper of the impact force $\%$. Because of this importance, several authors have studied and described its anatomy and mechanical properties. Striking was the difference in results on the mechanical properties in experiments that were done in vivo and in vitro. The in vivo tests that were conducted by way of impact experiments (e.g. ${ }^{10,11}$ ), gave energy losses up to $95 \%$, peak force values of $800 \mathrm{~N}$ and a heel pad stiffness of $150 \mathrm{kN} / \mathrm{m}$ at one bodyweight loading. The in vitro tests were conducted on an isolated part of a cadaver heel using a material testing machine (the 'Instron') (e.g. ${ }^{3}, 12$ ). In vitro, the energy loss was only about $30 \%$; peak force values were about $1800 \mathrm{~N}$, and heel pad stiffness $900-1500 \mathrm{kN} / \mathrm{m}$ at one bodyweight loading. Aerts et al. (1995) called the discrepancy between the in wivo and in vitro data "the heel pad paradox. They could contribute part of the differences to the way the two types of experiments were conducted. As an explanation of the remaining differences, the presence of a lower leg in the in vivo testing was suggested. Pain et al. (2001) made this suggestion plausible by studying the effect of the soft tissue of the lower leg using a two-dimensional mathematical model of the shank and heel pad. We hypothesized that the absence of a functional venous plexus in the heel pad in the in vitro experiments might be another explanation.

This hypothesis is substantiated by several other observations. In the intradermal and subcutaneous tissue of the sole of the foot, an extensive plexus of veins is present. At the swinging phase the plexus gets distended, at the stance phase the plantar pressure is ample to empty it. The impact force at heelstrike characteristically is of short duration. As shock damping is proportional to deformation, a hydro-mechanical action of the distended plexus could be expected. Moreover, the direct relation between the impact force and presence of blood in the heel is illustrated by the haemolysis that occurs in long-distance runners ${ }^{4,5}$.

The results of our study showed a small but significant decrease of the $F_{\max }$ at venous congestion. This made it plausible that the venous plexus did work as a hydro-mechanical shock absorber. The reduction of the $F_{\text {max }}$ was about $3 \%$ at 0.2 and $2 \%$ at $0.4 \mathrm{~m} / \mathrm{s}$. At $0.6 \mathrm{~m} / \mathrm{s}$ a damping effect was no longer observed. This frequency dependence also corroborated the hypothesis, because the damping properties of a system are related to the rate of deformation. The vertical touch-down velocities at locomotion are highly variable, interindividually as well as intraindividually ${ }^{8}$. In one study, the mean value of the vertical velocities in 10 subjects was $0.70 \mathrm{~m} / \mathrm{s}$, ranging from 0.16 to 1.2 at a running velocity of $3.6 \mathrm{~m} / \mathrm{s}^{13}$. In another study, touch-down velocities ranged from 0.8 to $1.2 \mathrm{~m} / \mathrm{s}$ at a running velocity of $3.5 \mathrm{~m} / \mathrm{s}^{14}$. Therefore, the impact velocities of the present study are representative for walking. The results suggest that in most of the individuals a filled venous plexus would work during walking, but would loose its effect during running. The Stiffness merely showed a tendency to decline at congestion, and to rise at decongestion. $D_{\max }$ and $E_{\text {dls }}$ did not show any significant effect of venous congestion. The most probable reason why these three parameters did not show a significant effect is that the error of the measurements is only slightly smaller than the effect we tried to measure.

The observed effects might be an underestimation. First of all, in the average adult more than $1 \mathrm{~m}$ water pressure distends the veins of the foot during locomotion. In our experiment, the induced venous congestion lasted for two and a half minutes only. Secondly, the only practical way to induce a relative empty venous plexus at the start of the measurements was to raise the leg above the level of the heart. Although we applied this measure, we do not know whether it was sufficient. Finally, as reported elsewhere (Weijers et al., simultaneously submitted), the repetitive character of the impacts had an effect of its own on the mechanical properties of the heel pad (Fig. 3). To take this effect into account, we averaged the trials of each stage of both the congested and the dummy series. As illustrated in Figure 3 , the magnitude of the effect of (de)congestion was not constant within the different stages. Thus, averaging reduced the noise at each stage at the expense of an underestimation of the effect of (de)congestion. 
Even though some underestimation of the effects of venous congestion might have occurred, the effects would remain relatively small. However, the repetitive character of locomotion makes that it probably contributes to damping in a valuable way. Even in walking, a peak transient deceleration of the tibia of approximately $2-7 \mathrm{~g}$ can be reached at heel strike ${ }^{1.15}$. Up to what extent the observed effect plays a role in patho-physiology remains to be seen.

Initially we planned to measure impacts of $0.2-1.0 \mathrm{~m} / \mathrm{s}$. However, when the impact velocity exceeded $0.6 \mathrm{~m} / \mathrm{s}$ (equal to an input energy of $0.9 \mathrm{~J}$ ), the impact gave such discomfort over the lateral condyle that we gave up the 0.8 and $1.0 \mathrm{~m} / \mathrm{s}$ trials. Theoretically, a subject should tolerate an input energy of at least $3.5 \mathrm{~J}$ easily. Cavanagh (1984) and Kinoshita (1993) reported on upper limits of subject tolerance of 2.1 and $3.2 \mathrm{~J}$ 10, 1.3. These authors used a contact area of 60 and $12.6 \mathrm{~cm}^{2}$, respectively, while we used $9.6 \mathrm{~cm}^{2}$. Moreover, in their experiments both condyles in the knee made contact with the supporting surface, while we only used part of the lateral condyle. Besides, in our study the soft tissue over the lateral femoral condyle was compressed between the cortex of the femur and the concrete wall with $70 \mathrm{~N}$ to avoid a damping side effect. All this will have contributed to the difference in the limit of tolerance. Conspicuously, our subjects complained of pain over the femoral condyle, not the heel pad region. This illustrated that the heel pad is a structure specialised to sustain the transient impact force at heelstrike.

To place the present study in the context of previous reports some additional remarks should be made. At heel strike, the kinetic energy, that has to be absorbed by the body to halt the descending motion of the foot, depends on the touch-down velocity and the effective mass of the foot. At the pendulum impact test, the equivalents are the impact velocity and the mass of the pendulum. The values of these two parameters varied considerably among studies. The estimates of the effective mass that is decelerated at heelstrike varied from 3.6 to $9.4 \mathrm{~kg}{ }^{16,17}$. The reported mass of the pendulum varied at least from $1.6^{9}$ to $11.6 \mathrm{~kg}$ 11. In the present study a pendulum of $5 \mathrm{~kg}$ was used. The range of impact velocities of the pendulum also varied, but as most reports addressed running-related matters, usually values of $1.0 \mathrm{~m} / \mathrm{s}$ or higher were used. We used impact velocities of $0.2-0.6 \mathrm{~m} / \mathrm{s}$. The combination of the mass of the pendulum and the impact velocity determined the energy input. It varied from $0.2 \mathrm{~J}^{3}$ to $6.5 \mathrm{~J}^{11}$, we used up to $0.9 \mathrm{~J}$. To make matters even more complicated, the reported contact surfaces of the impacting pendulum are also variable. For instance Jorgensen et al. used a contact area of $9 \mathrm{~cm}^{2}$ and Aerts et al. of $32.2 \mathrm{~cm}^{2}$ and Cavanagh et al. of $60 \mathrm{~cm}^{2}, 3,9,13$. We used a contact area of $9.6 \mathrm{~cm}^{2}$. From the foregoing, it is obvious that comparison of previous reports with the present one is not straightforward. However, this did not hamper the interpretation of our results because we looked for changes of parameters over time, not so much for the magnitudes of them.

In summary, the results of the present study supported the fundamental hypothesis that the venous plexus damps the impact force of the heelstrike. Its effect is limited although some degree of underestimation might have occurred. Most likely, the damping effect will work at walking, not at running.

\section{Acknowledgements}

We thank Martin van der Wolf and Emile Arnoldussen of the IDEE of the University Maastricht for their technical support. 


\section{References}

1. Collins 13 and whittle MW: Impulswe forces during walkng and tweir chincal implications. Cin. Biomech. 1989; 4: 179-187.

2. Weijers RE, van Mameren H, Wallenkamp GH, et al.: Changes of the soft tissue of the forefoot during loading: volumetric study: Foot 2003; 13: 70-75.

3. Aerts $P_{*}$ Ker $R F$, De Clerca $D$, et al: The mechanical properties of the human heel pad: a paradox resolved. J Biomech 1995; $28 ; 1299-1308$.

4. Dang $\mathrm{CV}$ : Runner"s anemia, Jama 2001; $286: 714-716$.

5. Telford $R D, S l y, G$, Hahn $\mathrm{AG}_{\mathrm{g}}$ et al: Footstrike is the major cause of hemolysis during rurining. I. Appl PHysiol 2003; 94:38-42.

6. OBrier PC: Procedures for comparing samples with multiplie endpoints. Biometrics 1984; 40: 1079-1087.

7. Valiant GA: Transmission and attemuation of heelstrike accelarations, in Blomechanics of distance running, ed by Cavanagh PR. Human Kinetics Publistiers, Inc, Champaign, Illinois; 1990. 225-247.

3. Whittle MW: Generation and atteruation of transient impulsive forces beneath the foot: a rewlew. Gat Posture 1999; 10: 264-275.

9. Jorgensen $U$ and Bojsen-Moller F" Shock absorbency of factors in the shoe/heel interaction-with special focus on role of the heel pad. Foot Ankle 1989; 9: 294-299.

10. Kinoshita $H$, Ogawa $T$, Kuzuhara $K_{\text {, }}$ et al.: In viwo examination of the dynamic properties of the human heel pad. Int J Sports Med 1993; 14: 312-319.

11. Aerts $P$ and De Clerch D: Deformation characteristics of the heel region of the shod foot during a simulated heel strike: the effect of varying midsole hardness. I Sports Sci 1993; 11: 449-461.

12. Bennett $M B$ and Ker RF: The mechanical properties of the human subcalcaneal fat pad in compression. J Anat 1990; 171: 131-138.

13. Cavanagh PF, Valiant $\mathrm{GA}$ and Misewich $\mathrm{KW}$ : Biological aspects of modeling shoe/ foot interaction during funning, in Sport shoes and playing surfaces, ed by Frederick CA. Human Kinetics Publishers, Inc., Champaign, Illinols; 1984. 24-46.

14. Nigg BM: Experimental techniques in running shoe research, im Biomechanics of running shoes, ed by Nigg BM. Human Kinetics Publishers, Inc., Champaign, Illinois; 1986. 27-61.

15. Maclellan GE: Skeletal heel strike transcients, measurement, implications, and modification by footwear, in Sport shoes and playing surfaces, ed by Frederick CA. Human Kinetics Publishers, Inc., Champaign, Illinois; 1984. $76-86$.

16. Nigg BM: Factors influeneing kinetic and kinematic variables in riunning, in Biomechanics of running shoes, ed by Nigg BM. Human Kinetics Publishers, Inc, Champaign, Illinois; 1986. 139-159.

17. Ker RF, Bennett MB, Alexander RM, et al.: Foot strike and the properties of the human heel pad. Proc Inst Mech Eng [H] 1989; 203: 191-196. 


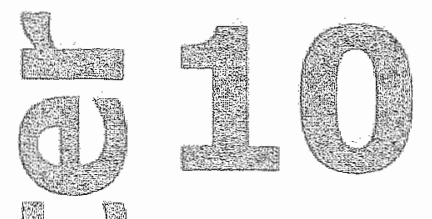

\section{Pendulum impact testing on the forefoot: a comparison with the heel}

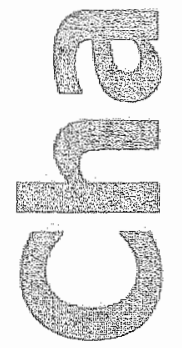

RE Weijers, AGH Kessels, and GJ Kemerink

Submitted

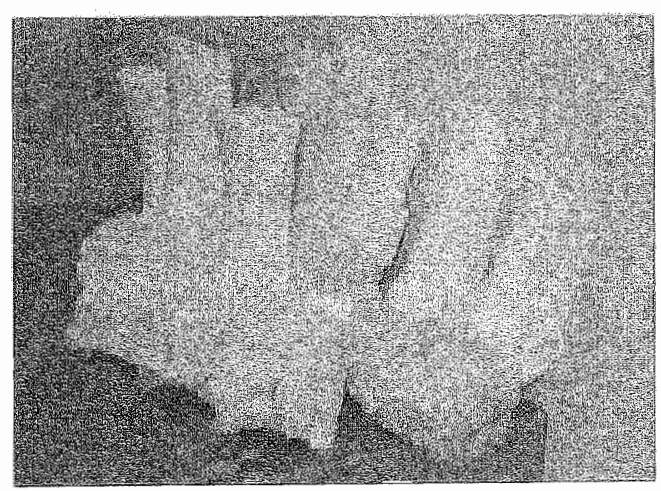




\begin{abstract}
The mechanics at the heel pad during locomation has been extensively investigated and yet it is not fully understood. As the anatomy and functian of the heel region and the forefoot differ, differences in mechanical behaviour could be expected. To our knowledge, presently no data are available on the forefoot. Therefore, we conducted pendulum impact tests on the forefoot similar to previous experiments on the heel with one and the same group of eleven subjects. The goal of the present study was to explore whether the forefoot also showed effects of venous congestion and repeated impacts as observed in the heel region. Moreover we wanted to compare the magnitude of the parameters that described the impacts on the heel region with thase on the forefoot. Of all subjects, two sequences of 18 impacts each were acquired at impact velocities of $0.2,0.3$, and $0.4 \mathrm{~m} / \mathrm{s}$. One sequence was used to explore the effect of venous congestion. This sequence was divided in three physiologically different, consecutive conditions: (i) a relatively empty venous plexus, (ii) a congested venous plexus and (ii) a decongested venous plexus. The other sequence was executed without lintervention and was used to analyse the effect of repeated impacts. The results did not show a significant contribution of venous congestion to the damping abilities of the sole. The effect of repetitive impacts was confirmed and results paralleled those of the heel. The mechanical characteristics were significantly different at the forefoot compared to the heel region. Most likely, the differences in anatomy of the forefoot and the heel explain this result rather than an intrinsic difference in the mechanical properties of the soft tissue of the sole of the foot. The results illustrate that the damping of an impact is not a llocal phenomenon, but involves the underlying structures as well.
\end{abstract}




\section{Introduction}

The heel pad is the most impoitant damper of the impact force during locomiotion ${ }^{1}{ }^{2}$, Several authors described its mechanical properties with a variety of impact tests under conditions comparable to running. At impact velocities similar to the descending velocity of the foot at walking, the behaviour of the heel pad was more complex, as recently reponted. The venous plexus of the sole modestly contributed to the damping properties of the heel pad ${ }^{3}$. Moreover, repeated impacts also influenced the mechanical properties " In contrast to the heel, the forefoot not only contains a superficial, subcutaneous venous plexus, but also deep veins ${ }^{5}$. The primary goal of this study was to explore the effect of congestion of the venous structures and repeated loadings on the damping power of the forefoot. of the forefoot, the mechanical properties of the padding were not reported yet. As the heel differs from the forefoot in several aspects, we wondered whether the mechanics of the two reglons also differed. To answer this second question we compared the data from the present experiment with those of our previous studies on the heel pad in one and the same group of subjects. The data may provide more insight in the mechanics of locomotion.

\section{Materials and methods}

\section{Principles of the experiments}

An instrumented pendulum impacted the sole of the forefoot with a chosen kinetic energy. After a double integration of the deceleration signal of a single impact, the load-deformation curve of that impact was constructed (Fig. 1). From this curve the magnitude of the maximum force $\left(F_{\text {max }}\right)$, maximum compression ( $D_{\text {max }}$ ), Stiffness and energy dissipation ( $E_{d i s}$ ) of each impact was calculated. By studying the changes in the magnitude of the parameters over time after appropriate intervention, the effects of venous congestion and of repeated impacts could be explored. The effect of the venous plexus was investigated by comparing pendulum impact experiments on empty veins with experiments on blood filled veins.

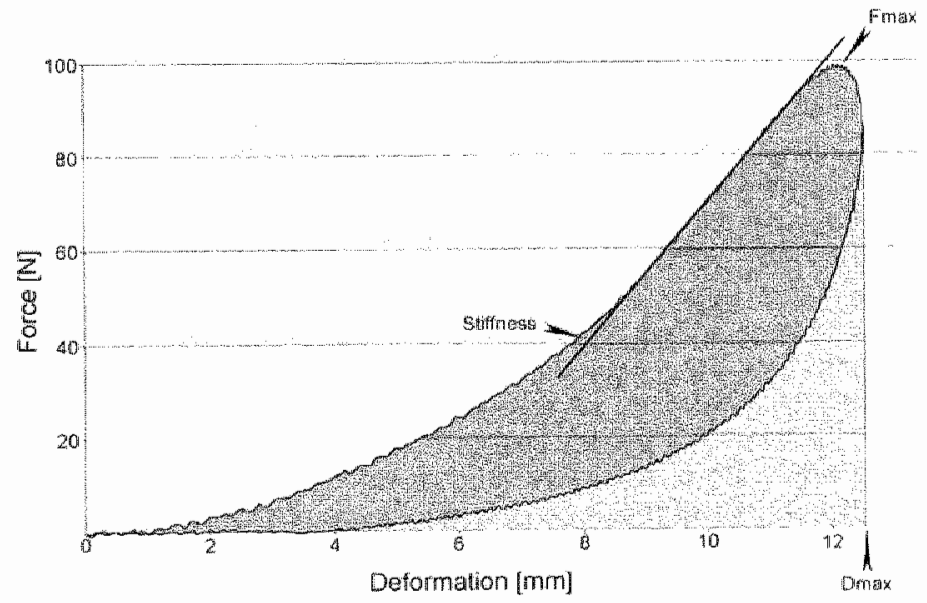

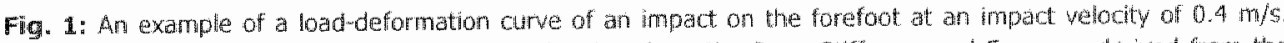

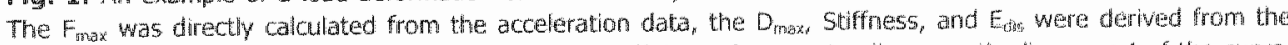
curve. The Stiffress was defined as the regression coefficient of regression line over he linear part of the curve. The $\mathbb{E}_{\text {was }}$ waned as: the dark grey area $100 /$ (light grey plus dark grey area). 
A relatively empty venous plexus was obtained by positioning the volunteer supine on the floor with his lower leg above the level of the body. Filling of the venous plexus was realised with an inflated cuff of a sphygmomanometer around the call. To measure the effect of repeated impacts a sequence of 18 consecutive impacts was measured. The results of this study were compared with the results of previous studies on the heel in the same subjects. For additional details on the execution and calibration of the experimental set-up we refer to previous studies ${ }^{3,4}$.

\section{The pendulum}

The pendulum consisted of a cylindrical steel bar suspended by cords in a frame on wheels. The bar had a length of $67.0 \mathrm{~cm}$, a cross section of $9.62 \mathrm{~cm}^{2}$ and a mass of $5.1175 \mathrm{~kg}$. The effective length of the suspending cords was $1.5025 \mathrm{~m}$; from each end of the bar two cords were connected to points on the frame that were $30 \mathrm{~cm}$ apart in the lateral direction. The steel bar was instrumented with a mono-axial accelerometer (model PB3ACW, Oceana Sensor, Virginia Beach, VA) with a nominal sensitivity of $100 \mathrm{mV} / \mathrm{g}$, with $\mathrm{g}$ the gravitational constant $\left(9.82 \mathrm{~m} / \mathrm{s}^{2}\right)$. The frequency response was within $\pm 5 \%$ from $1 \mathrm{~Hz}$ to $4 \mathrm{kHz}$. The actual measurements of the impacts had to be corrected for the (single pole) response fall-off at low frequencies; this was done in the frequency domain. Corrections to be applied due to high frequency fall-off were negligible. The signal of the accelerometer was digitised using a multifunction PC board (AT-MIO-16E-10, National Instruments, Austin, TX, USA). This board had an adjustable gain amplifier and a 12-bit analog to digital converter. The sample rate during data acquisition was $5 \mathrm{kHz}$; the duration of the sampling period was 5 seconds. Data correction (low-off compensation) and data analysis were done in Matlab version 6.1. (The MathWorks Inc., Natick, MA, USA). The calibrations of the accelerometer resulted in a sensitivity of $99.0 \mathrm{mV} / \mathrm{g}$ and a time constant of $0.53 \mathrm{~s}$ for the low frequency roll-off. Both values were close to the nominal values specified by the manufacturer ( 99 $\mathrm{mV} / \mathrm{g}, 0.5 \mathrm{~s})$.

\section{Subjects}

The subjects were healthy males, without physical complaints of their feet. No plantar callus formation was present. Eleven subjects were included (mean age: 38.1 years, range: 29-54; mean weight: $79.1 \mathrm{~kg}$, range: $70-89$; mean length: $1.82 \mathrm{~m} ;$ range: $1.74-1.93$ ). Our Medical Ethic Committee approved the study. All subjects gave informed consent.

\section{Position of the subjects and stabilization of the foot}

The subjects lay supine on the ground beside a massive rectangular concrete pillar. The hip and knee were flexed over $900^{\circ}$. A cuff of a sphygmomanometer was wrapped around the uncovered lower leg just below the knee. The stabilization of the foot posed a special problem. To reliably measure the force during the impact, a rigid support was required. Moreover, at the same time the dorsum of the foot should be supported to avoid dorsiflexion of the foot during impact. Therefore, we developed the following approach. In preparation to the experiment, we first made a plaster cast of each volunteer covering the dorsum of the left foot and the anterior side of the lower third of his lower leg. Then, we moulded a truly flat surface on the dorsal part of these casts at the region of the forefoot, in such a way that it formed a stablle interface between the foot and a wood block $(30 * 10 * 10 \mathrm{~cm})$ that was rigidly fixed to the concrete pillar. Two elastic slings pulled the foot and cast together against the block. To secure this position, the foot and cast were place in a homemade "vice" (Fig. 2). 

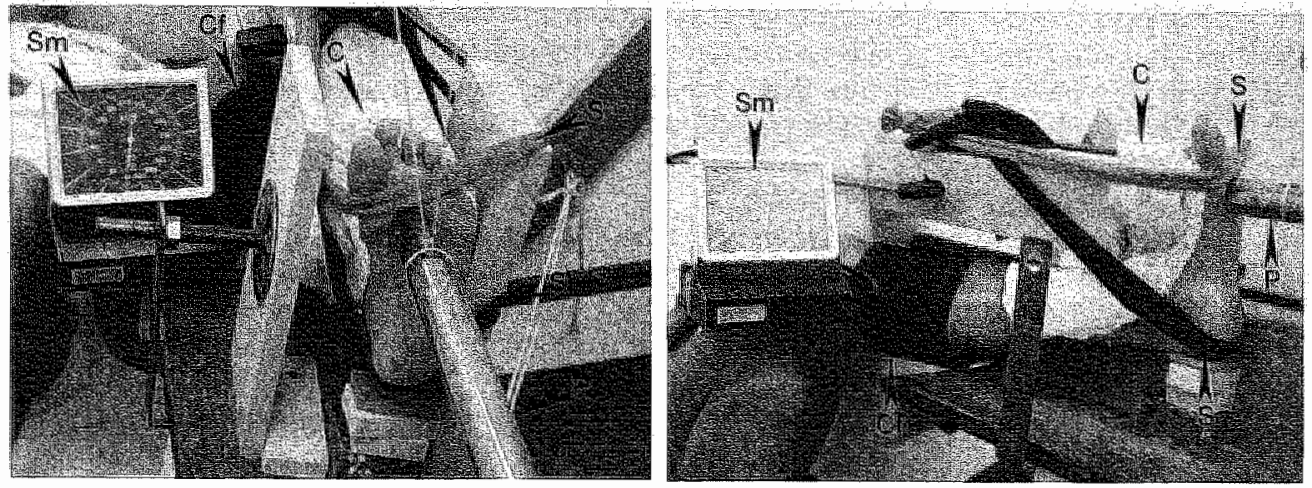

$A_{4}$

B

Fig. 2: A wew from above (A) and a view side long (B) of the position of the foot and the perndulun during the experment. For the sake of clarty we removed the 'jaw' of the vice in $(8)$. (C: cast; cr: cuff; pi pendulum; S: elastic silng: Sm= sphygmomanometer)

\section{The centre of impact}

Prior to the positioning of the pendulum, the fibular sesamoid, the first, and the second metatarso-phalangeal joints were located by palpation and marked on the skin. These markings were to be positioned on the edge of the circle that formed the impact area of the bar of the pendulum. Thus, the centre of impact was positioned over the first intermetatarsal space and proximal to the first and second metatarso-phalangeal joint. After the pendulum was aligned, its bearing structure was clamped between two walls to prevent oscillation of the frame. In equilibrium, the centre of the end of the bar just touched the marked skin.

\section{The measurement protocol}

We executed the measurements at three impacts velocities: $0.2,0.3$ and $0.4 \mathrm{~m} / \mathrm{s}$. The out of equilibrium amplitude of the bar at the moment it was released, was used to adjust the velocity of the impacting bar. At each velocity, we measured a sequence of 18 impacts: six minutes, at a rate of three impacts per minute; the dummy procedure. Between two sequences, we checked the alignment of the leg, foot and bar and adjusted the amplitude of the pendulum. This took about two minutes.

To evaluate the effect of venous congestion, a sequence of 18 impacts was divided into three stages, the congested procedure. In Stage 1 , three baseline impacts were made. Next, the cuff around the lower leg was inflated to $80 \mathrm{mmHg}$ to induce venous congestion. For a period of two and a half minutes the cuff remained inflated (Stage 2, 8 impacts). Finally, the cuff was deflated and we continued measuring for another two and a half minutes (Stage 3,7 impacts). The congested and the dummy procedure were measured at the same three impact velocities $(0.2,0.3$, and $0.4 \mathrm{~m} / \mathrm{s})$. We separately executed the dummy and the congested procedure in random order. Both procedures were completed within one day.

\section{Definition of the parameters}

By means of a double integration of the deceleration signal, a load-deformation curve of each impact was constructed. This procedure was previously described ${ }^{3,4}$. From the curve the foliowing parameters were calculated (Fig.1):

- $F_{\text {max: }}$ maximun Force (= mass of pendulum * maximum acceleration) [N]

- $D_{m i x}:$ maximum compression [mm]

- Stiffness: the coefficient of the linear regression equation of the linear part of the loadcompression curve $[\mathrm{kN} / \mathrm{m}]$ 
- Edy dissipated energy: the lost energy expressed as a percentage of the kinetic energy that the pendulum had at the beginning of the impact. It is defined as; (area under the load-deformation curve during compression minus the area during recoil)*100/ area under the compression part of the load-deformation curve.

\section{Statistical analysis}

For the statistical analysis of the effect of (i) venous congestion, (ii) the effect of repeated impacts and (iii) the comparison of the magnitude of the parameters between the forefoot and the heel, we used SPSS 10 (SPSS Inc, Chicago, Illinois; USA) software.

\section{(1) The effect of venous congestion}

To analyse the effect of venous congestion, the averages of the measurements in Stage 1 , Stage 2, and Stage 3 of a sequence were calculated. To find the gross effect of the congestion, the average of Stage 1 was subtracted from Stage 2. To find the gross effect of decongestion, we subtracted Stage 2 from Stage 3. To correct for possible effects of repeated impacts, we treated the data of the dummy procedure the same way. The outcomes of the conglested (the gross effect) and the dummy procedure (the correction) of each volunteer were paired (Fig. 3). For each parameter, this algorithm was applied to the data of all volunteers at the three impact velocities. Finally, the null hypothesis, that there were no effects of venous congestion (difference between the means of Stage 1 and 2) or decongestion (difference between the means of Stage 2 and 3), was tested. Two tests were used:

- the two-tailed student t-test on all four parameters at all three impact velocities.

- the ordinary least squares (OLS) test as described by O'Brien and modified by Lauter ${ }^{6}$. In this test the outcomes of the three impact velocities were pooled for each parameter to increase the power. It gives the overall tendency of the parameter and the p-value.

\section{Congested procedure Dummy procedure}

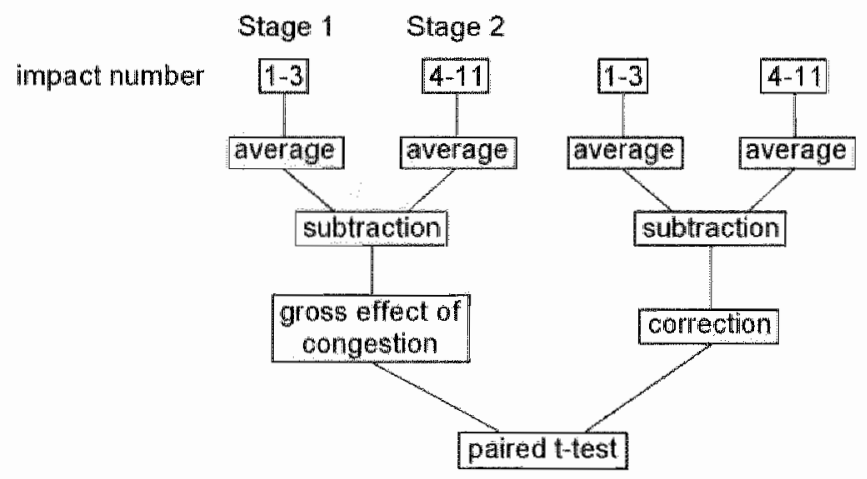

Fig. 3: Schematic representation of the analysis of the effect of congestion. This flow chat was followed for each subjef and parameter at all the three impact velocties. The analysis of decongestion (stage 2, impact $4-11$ and stage 3 impact 12-18) was done in an identica way.

(ii) The effect of repeated impacts

To analyse the effect of repeated impacts, we fitted an exponential function on sequences of 18 consecutive impacts of the dummy procedure. The general form of this function was $f(n)=a+b^{*} e^{e^{*} n}$, with ' $n$ ' the $n^{\text {th }}$ impact of a sequence. Next, we calculated the relative 
change between the first and the $18^{\text {th }}$ impact: $\left.(f(18)-f(1))^{*} 100 / f(1)\right)$. This was done for all four parameters at all three impact velocities and for each subject. Finally, these relative changes were statistically analysed with the one sample t-test, for each parameter at each velocity separately, the OLS-test for each parameter and the repeated measure ANOVA-test for each parameter across the velocities.

\section{(iii) The comparison between forefoot and heel}

To test the differences in the magnitude of $F_{\text {maxx }} D_{\text {miax, }}$ Stiffness, and $E_{\text {dis }}$ between the forefoot and the heel, we used the two-tailed student t-test on the $18^{\text {th }}$ value of the fitted curves of both dummy procedures for each subject at all impact velocities.

\section{Precision of the measurement}

Two aspects of the precision of a measurement can be distinguished: its reproducibility and its accuracy. Reproducibility is most important for the analysis of the effects of the venous congestion and the repeated impacts, accuracy for the analysis of the differences between the magnitude of the parameters at the forefoot and the heel. As previously explained ", the mean standard deviation (calculated as the square root of the mean of the variances of all the subjects) of the maximum velocity $\left(v_{\max }\right)$ and the kinetic energy $\left(E_{k i n}\right)$ can be defined as a standard of reproducibility of respectively the first and second integration. These were 0.016 , 0.020 , and $0.024 \mathrm{~m} / \mathrm{s}$ (respectively $6.9,5.7$, and $4.7 \%$ ) for the first integration and 0.018 , 0.020 , and $0.024 \mathrm{~J}$ for the second integration at $0.2,0.3$, and $0.4 \mathrm{~m} / \mathrm{s}$ impact velocity.

To indicate the accuracy, we calculated the InterClass Correlation (ICC) for each parameter at all velocities. The ICC expresses the subject explained variance as a percentage of the total variance of the observations. The ICC of the $F_{\text {max }}$ were $0.978,0.989$, and 0.997 , of $D_{\max } 0.810,0.903$, and 0.906 , of Stiffness $0.934,0.986$, and 0.994 , and of $E_{\text {ats }} 0.654,0.667$, and 0.798 at $0.2,0.3$, and $0.4 \mathrm{~m} / \mathrm{s}$ respectively. The ICC of the heel were only marginally better. For data on the ICC of the experiment of the heel we refer to previous work ${ }^{4}$.

\section{Validation of the comparison}

A comparison of the fore foot and the heel was one of the objectives of this study. It was reported that the supporting surface of the impacted body could influence the magnitude of the parameters of the impact ${ }^{7}$. In the heel experiments, a concrete pillar supported the knee and lower leg. In the fore foot experiment a combination of a plaster cast and a block of wood was rigidlly interposed between the forefoot and the concrete pillar. To evaluate a possible effect of the difference in set-up of the experiments we did additional validation experiments. At each impact velocity, the pendulum collided with a $2 \mathrm{~cm}$ thick felt layer that was successively attached to the pillar and to the combination of the plaster cast and the block of wood fixed to the pillar. At all three impact velocities, the means of the values of the parameters of five impacts at each condition were calculated. The results only showed a consistent and significant lower $F_{\max }$ on wood/ plaster than on the pillar of $1.8,1.3$ and $2.1 \mathbb{N}$ $(2.9,1.1$ and $1.1 \%$ ) at respectively $0.2,0.3$ and $0.4 \mathrm{~m} / \mathrm{s}$ impact velocity ( $\mathrm{P}$ values: <.001, .02 and .04 using a Student $t$-test). The results on the other parameters were not significant.

\section{Results}

The results of the effects of venous (de)congestion were summarized in Figure $4 \mathrm{~A}$ and $\mathrm{B}$. Although the results suggested a consistent decrease in $F_{\text {max }}$, Stiffness, and $E_{\text {dis }}$ at all impact velocities at congestion, and an increase in $F_{\text {пах }}$ and Stiffness at decongestion, the results were not significant. Also the OLS-test on the pooled data did not show a significant result (Table 1). Compared with the heel region, the effects were-generally speaking- of the same magnitude (Table 2). 

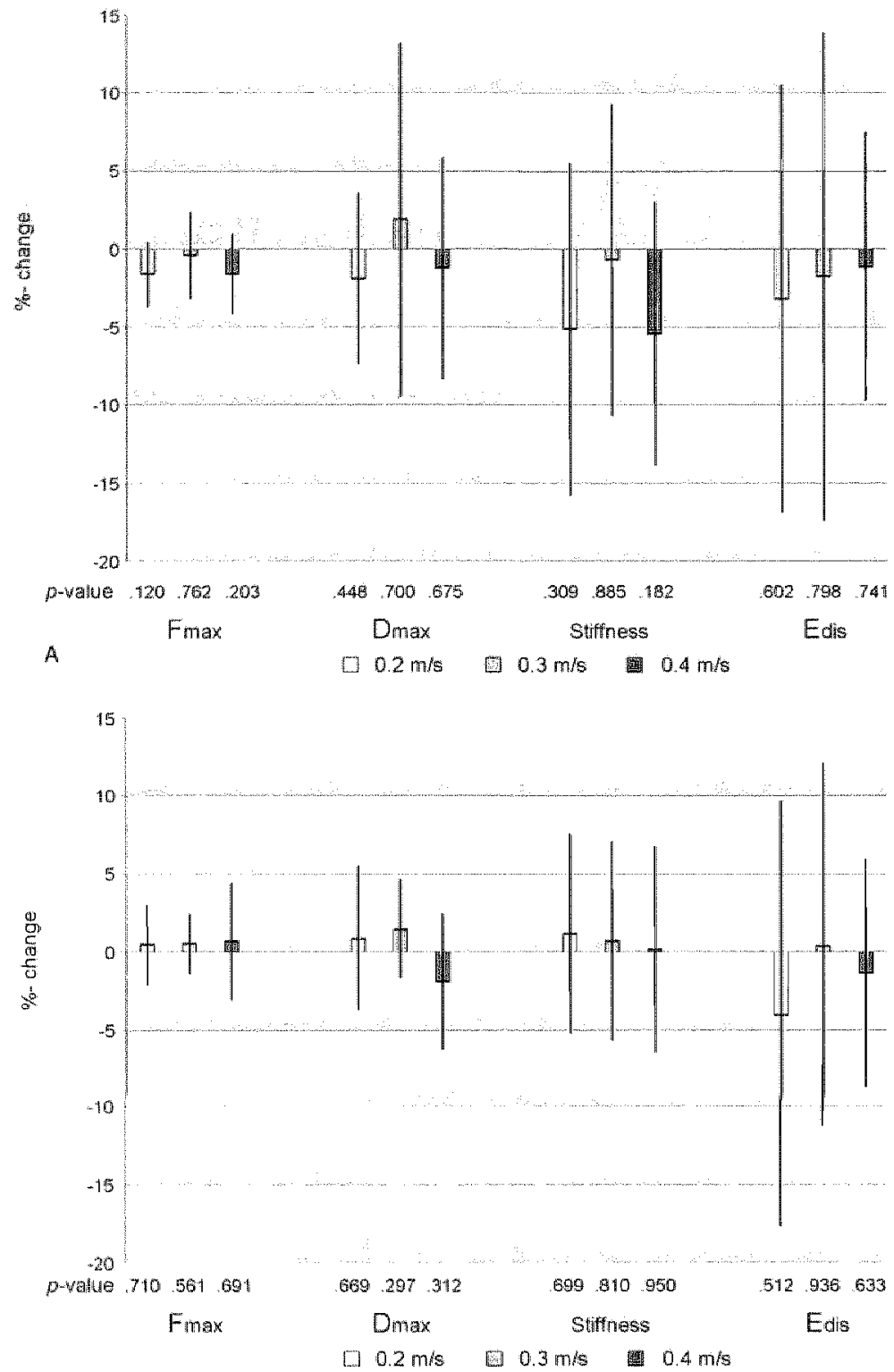

B

Figl. 4: The efrect of (de) congestion. The mean differences, the 95\%-confidence interval and the $p$-value of all the parameters at the three impact velocities at congestion (A) and decongestion (B) were depided. 


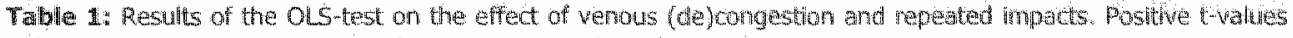
indicate an increase of the value of the parameter after interwention.

\begin{tabular}{lcccccc} 
& \multicolumn{2}{c}{ congestion } & \multicolumn{3}{c}{ decongestion } & \multicolumn{2}{c}{ repeated impacts } \\
\cline { 2 - 7 } & $\mathrm{t}$ & $\mathrm{p}$ & $\mathrm{t}$ & $\mathrm{p}$ & $\mathrm{t}$ & $\mathrm{p}$ \\
\hline $\mathrm{F}_{\text {max }}[\mathrm{N}]$ & -1.5 & 0.174 & 0.9 & 0.398 & 4.5 & 0.001 \\
$\mathrm{D}_{\max }[\mathrm{mm}]$ & -0.4 & 0.730 & 0.4 & 0.676 & -0.2 & 0.864 \\
Stiffness $[\mathrm{kN} / \mathrm{m}]$ & -1.1 & 0.283 & 0.3 & 0.737 & 3.7 & 0.004 \\
$E_{\text {dis }}[\mathrm{J} / \mathrm{J}]$ & -0.5 & 0.663 & -1.4 & 0.211 & -1.0 & 0.362 \\
\hline
\end{tabular}

Table 2: Comparson helween the results of the heel and the Forroot. The bffect of venous conguston ( 2 minus stage 1) * 100/ Stage 1), decongestion (Stage 3 minus 5tage 2) 100/Stage 2), and rapated inpatts

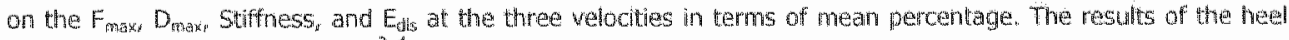
originated from previous studies ${ }^{3+\frac{4}{4}}$.

\begin{tabular}{|c|c|c|c|c|c|c|c|}
\hline & \multirow[b]{2}{*}{ velocity } & \multicolumn{2}{|c|}{ congested } & \multicolumn{2}{|c|}{ decongested } & \multicolumn{2}{|c|}{ repeated impacts } \\
\hline & & forefoot & heel & forefoot & heel & forefoot & theel \\
\hline$F_{\max }$ & 0.2 & -1.6 & -2.6 & 0.4 & 1.5 & 4.3 & 3.0 \\
\hline \multirow[t]{2}{*}[\mathbb{N}]{} & 0.3 & -0.4 & & 0.5 & & 1.9 & \\
\hline & 0.4 & -1.6 & -1.8 & 0.7 & 0.8 & 4.0 & $\mathbb{1 . 1}$ \\
\hline$D_{\max }$ & 0.2 & -1.9 & -1.7 & 0.9 & 0.2 & -0.4 & 1.6 \\
\hline \multirow[t]{2}{*}[\mathrm{mm}]{} & 0.3 & 1.9 & & 1.5 & & 5.0 & \\
\hline & 0.4 & -1.2 & 1.2 & -1.9 & -0.4 & -5.7 & 1.5 \\
\hline Stiffness & 0.2 & -5.1 & -1.3 & 1.1 & 1.7 & 4.3 & -0.2 \\
\hline \multirow[t]{2}{*}[\mathrm{kN}/\mathrm{m}]{} & 0.3 & -0.7 & & 0.7 & & 3.2 & \\
\hline & 0.4 & -5.4 & -3.7 & 0.2 & 2.5 & 8.5 & -3.1 \\
\hline$E_{\mathrm{dis}}$ & 0.2 & -3.2 & -1.5 & -4.0 & -0.2 & -1.7 & -1.8 \\
\hline \multirow[t]{2}{*}[\mathrm{J}/\mathrm{J}]{} & 0.3 & -1.8 & & 0.4 & & -3.4 & \\
\hline & 0.4 & -1.1 & 1.1 & -1.4 & 0.9 & -3.8 & -4.6 \\
\hline
\end{tabular}

The results of the effects of repeated impacts were summarized in Figure $5 . F_{\text {max }}$ and Stiffness increased on repeated impacts. For $F_{\text {max }}$, this was significant at 0.2 and $0.4 \mathrm{~m} / \mathrm{s}$ and for Stiffness at $0.4 \mathrm{~m} / \mathrm{s}$. Although $E_{\text {dis }}$ showed a decrease at all velocities, the results were not significant. The OLS-test showed a significant overall increase in $F_{\text {max }}$ and Stiffness (Table 1). Compared with the heel region the results on the Stiffness were opposed (Table 2). The results on the $D_{\text {max }}$ were indifferent and not significant. The ANOVA-test did not show a significant relation between the impact velocity and the magnitude of the changes of the parameters ( $p$-values of $F_{\max }$ Stiffness, $D_{\max ,}$ and $E_{\text {dis }}$ were $0.07,0.07,0.08$, and 0.80 , respectively). Nor did the results of the ANOVA-test suggest a trend across the impact velocities, as was already suggested in Figure 5. 


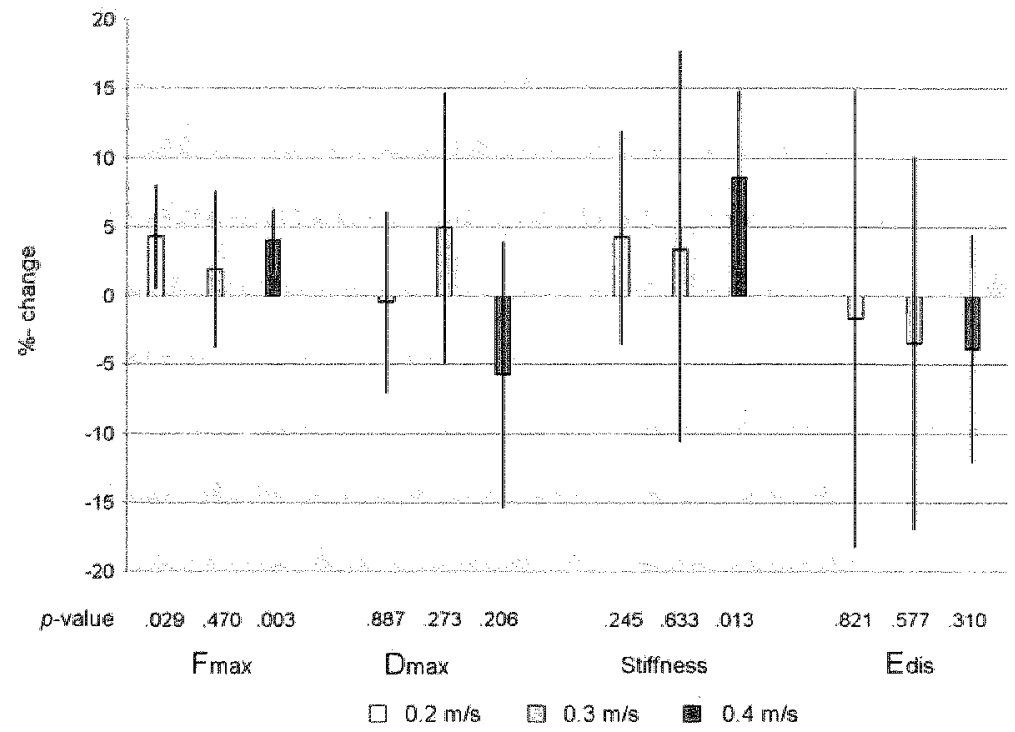

Fig. si: The effect of repeated impacts. The mean differences, the $95 \%$-confidence interval and the p-watue of all the parameters at the three inpact velocities were depicted.

Table 3: Comparison between values of the parameters at the forefoot and the heel. The p-values were the resuls of the student t-test on the paired values of a parametar at the forefoot and the theol in all subjects. The

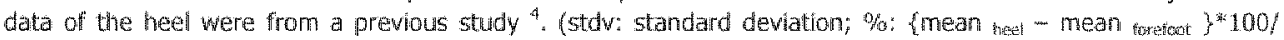
mean med.

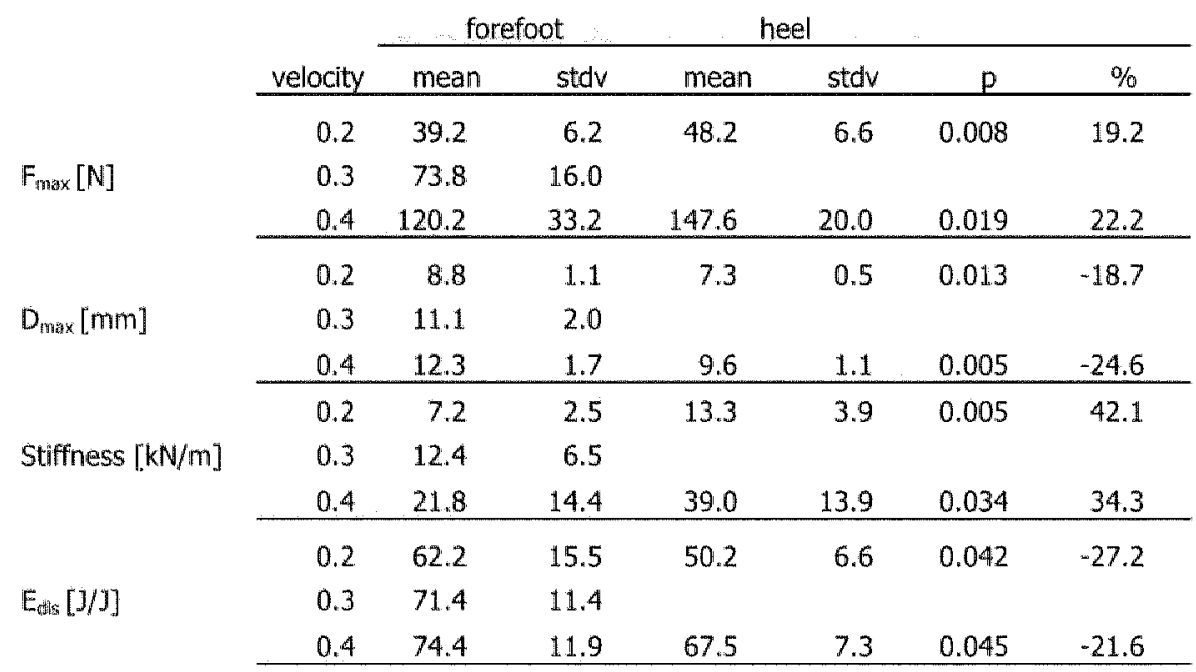




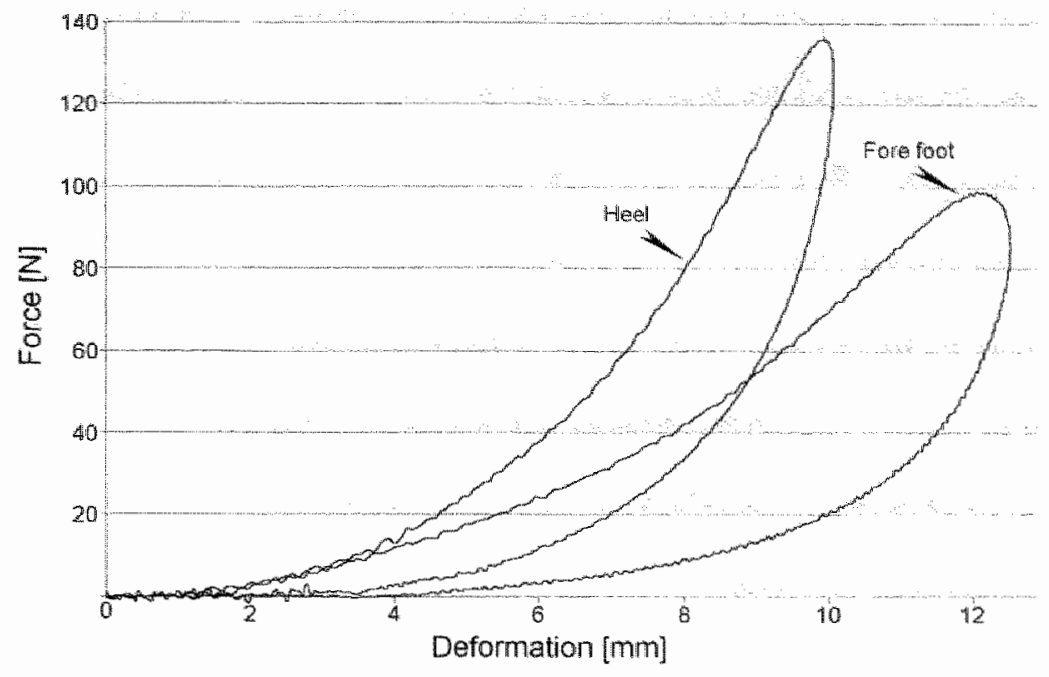

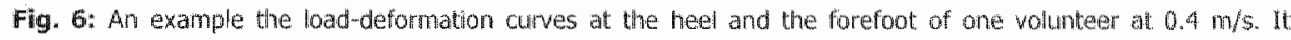
illustrates the differences of the characteristics.

The magnitude of the average values of the parameters was summarized in Table 3. The impact on the forefoot induced a $20 \%$ lower force than on the heel. The indentation of the soft tissue of the forefoot was about $20 \%$ deeper, the dissipated energy about $25 \%$ more and the Stiffness about $40 \%$ less. All differences were significant. The characteristic difference between an impact on the forefoot and on the heel were illustrated in Figure 6.

\section{Discussion}

In contrast to the heel, the forefoot did not show a significant effect of venous congestion. Repetitive impacts resulted in a significant increase of $F_{\max }$ and Stiffness. The magnitude of all parameters of the impact on the padding of the forefoot and the heel significantly differed.

In a previous study on the heel pad "the venous structures in the subcutaneous tissue showed to contribute only moderately to its damping power. However, in addition to the subcutaneous superficial venous structures similar to that of the heel pad, in the forefoot: also larger veins are positioned centrally in the foot ${ }^{5}$. The assumption that therefore the contribution of veins to the damping power would be bigger in the forefoot could not be confirmed by our results. Most likely, the reproducibility of the impact test was not sufficient: to detect the small effect of venous congestion in the forefoot. This may justify the conclusion that if there would be any effect at all, it was less than $5 \%$. The difficulities in stabilizing the foot probably caused the decrease in reproducibility compared to the heel.

Repeated impacts on the forefoot showed a significant increase in Fmax and Stiffness. This increase reflected the wisco-elastic properties of the sole of the foot ${ }^{4}$. Both parameters increased in magnitudie about $5 \%$ which is similar to the results on the heel region ". In contrast to the heel region, we could not detect a velocity dependence in the forefoot. In the heel region repeated impacts were associated with and increase of $F_{\max }$ of $3 \%$ at $0.2 \mathrm{~m} / \mathrm{s}$, $1.2 \%$ at $0.4 \mathrm{~m} / \mathrm{s}$ and a decrease of $2.1 \%$ at $0.6 \mathrm{~m} / \mathrm{s}$. We suggested that this observation reflected the adlaptation of the muscles of the calf to the impact force, also referred to as 
tuning ${ }^{-10}$. As the forefoot lacks such an additional possibility to damp the impact force, the consistent increase was in line with what could be expected. Another possible explanation for the lack of velocity dependence was that perhaps reproducibility of the measurements was insufficient to detect those relatively small effects.

The magnitude of the parameters that characterize the impacts on the forefoot and the heel significantly differed. $F_{\text {unax }}$ and Stiffness in the forefoot were smaller, whereas $D_{\max }$ and $E_{\text {dis }}$ were bigger. These differences could not be explained from the structure or thickness of the sole. At the heel, the basic pattern of compartments of fibro-elastic tissue filled fat cells is said to account for the damping properties ${ }^{11,12}$. The fibro-elastic sheets loosely connect the skin to underlying bone. Although different in detall, the padding of the forefoot resembles this pattern so much ${ }^{12,13}$ that it is not likely that this could account for a substantial part of the difference in the mechanical properties. As measured with ultrasound, the thickness of the padding at the heel was on average $16.5 \mathrm{~mm}$. At the forefoot, the average thickness was $12 \mathrm{~mm}$ in another group of subjects ${ }^{14}$. This difference in thickness would rather lead to a lower than the observed higher damping power at the forefoot.

Although the pendulum collided with the skin, the impact would also be influenced by the deeper layers of the foot and in case of the heel even the presence of a lower leg. In view of the foregoing, it was therefore likely that the differences in magnitude came from the deeper lavers. At the forefoot, a constellation of more or less mobile metatarsals and toes support the sole, while at the heel it is a single, rigid bone. As suggested earlier ${ }^{15}$, this essential difference in anatomy could explain the difference in damping power. During the loading of the forefoot the metatarsals shift: the medial longitudinal arch lowers and the metatarsals splay ${ }^{16}$. By itself, this will dissipate energy. Moreover, as the intermetatarsal spaces widen, they provide a gateway from the plantar to the dorsal side of the foot. During the relatively rapid loading of the forefoot during locomotion, plantar soft tissue can move dorsally in the intermetatarsal space at the cost of energy. At the heel, the padding is supported by the calcaneus, lacking the additional possibilities of energy dissipation.

Some remarks on the design of study should be made. We intended to position the centre of the region of impact over the first intermetatarsal space, as the first is the widest of all intermetatarsal spaces. We had to compromise because in that case the fibular sesamoid would be within the region of impact. The choice of the location in antero-posterior direction was made because the deep veins are most abundant proximal to the MTP-joints. Furthermore, the setting of the experiments on the forefoot and the heel were not identical. The validation test showed that maximally $2 \%$ of the difference in the magnitude of the impact on the fore foot and the heel could be attributed to the difference in set-up. This $2 \%$ is far less than the observed differences between the forefoot and heel.

We tested the forefoot up to $0.4 \mathrm{~m} / \mathrm{s}$ only. Because this appeared to be the upper limit of tolerance, we could not compare the forefoot with the heel at $0.6 \mathrm{~m} / \mathrm{s}$. At the heel region the tolerance limit was $0.6 \mathrm{~m} / \mathrm{s}$. Physiologically seen, this is not surprising, because at normall locomotion the vertical landing velocity at the heel is higher than at the forefoot in most individuals.

The measurements on the forefoot were less accurate than on the heel. Likely a combination of factors caused this. Although we paid a lot of attention to stabilize the forefoot, we did not succeed as well as at the heel. Moreover, the surface of the heel is flatter than that of the ball of the foot. This will cause a somewhat asymmetrical impact, giving unaccountable loss of energy. This subject was elucidated elsewhere ${ }^{4}$.

In conclusion, the mechanical characteristics were significantly different at the forefoot compared to the heel region. It was more probable that the difference in anatomy explained this rather than the intrinsic mechanical properties of the soft tissue of the sole of the foot. 
This illustrated that the damping of an impact is not a strictly local phenomenon, but involves the underlying structures as well. The effect of repetitive impact was confirmed and paralleled that at the heel. The hypothesis of the venous congestion contributing to the damping ability of the sole could not be confirmed.

\section{References}

1. Jorgensen $U$ and Bojsen-Moller $F$ : Shock absorbency of factors in the shoe/heel interaction with special focus on role of the heel pad. Foot Ankle 1989; 9: 294-299.

2. Kinoshita $H$, Ogawa $T$, Kuzuhara $K$, et al.: In vivo examination of the dynamic properties of the human heel pad. Int I Sports Med 1993; 14: 312-319.

3. Weijers RE, Kesseis AGH and Kemerink GJ: The damping properties of the wenous plexus of the heel region of the foot during simulated heelstrike. $J$ Biomech in press in press;

4. Weijers RE, Kessels AGH and Kemerink G]: The effects of repeated low velocity impacts on the mechanical properties of the heel pad. I Biomech resubmitted resubmitted;

5. Saraffian SK: Chapter 7: Angiology, in Anatomy of the Foot and Ankle, ed by Saraffian SK. Lippincot J.B., Philadellphia; 1993. 89-294-356.

6. O'Brien PC: Procedures for comparing samples with multiple endipoints. Biometrics 1984; 40: 1079-1087.

7. Aerts $\mathrm{P}, \mathrm{Ker} \mathrm{RF}_{j}$ De Clercq $\mathrm{D}$, et al.: The mechanical properties of the human heel pad: a paradox resolved. J Biomech 1995; 28: 1299"1308.

8. Wakeling JM and Nigg BM: Modification of soft tissue vibrations in the leg by muscular activity. J Appl Physiol 2001; 90: 412-420.

9. Wakeling JM, Von Tscharner $V_{f}$ Nigg BM, et al.: Muscle activity in the leg is tuned in response to ground reaction forces. J Appl Physiol 2001; 91: $1307-1317$.

10. Nigg BM and Wakeling JM: Impact forces and muscle tuning: a new paradigm. Exerc Sport Sci Rev 2001; 29: $37 \times 41$.

11. Jahss $\mathrm{MH}_{r}$ Michelson $\mathrm{JD}$, Desal $\mathrm{P}_{\mathrm{r}}$ et al.: Investigations into the fat pads of the sole of the foot: anatomy and histology. Foot Ankle 1992; 13: $233-242$

12. Saraffian 5K: Chapter 10: Functional anatomy of the foot and ankle, in Anatomy of the Foot and Ankle, ed by Saraffian SK. Lippincot J.B., Philadelphia; 1993. 89-294-356.

13. Bojsen-Moller $F$ and Flagstad KE: Plantar aponeurosis and intermal architecture of the ball of the foot. $J$ Anat 1976; 121: 599-611.

14. Weijers RE, Walenkamp GHIM, Kessels AGH, et all.: Plantar pressure and sole thickness of the forefoot. Foot and Ankle Int 2004; in press:

15. Weijers $R E$, van Mameren $\mathrm{H}_{*}$ Walenkamp $\mathrm{GH}_{\text {, }}$ et al.: Changes of the soft tissue of the forefoot during loading: a wolumetric study. Foot 2003; 13: 70-75.

16. Shereff $M J$, DiGiowanni $L$, Bejgani $F J$, et al.: A comparison of noinweight bearing and weight-bearing radiographs of the foot. Foot Ankie 1990; 10: 306-311. 

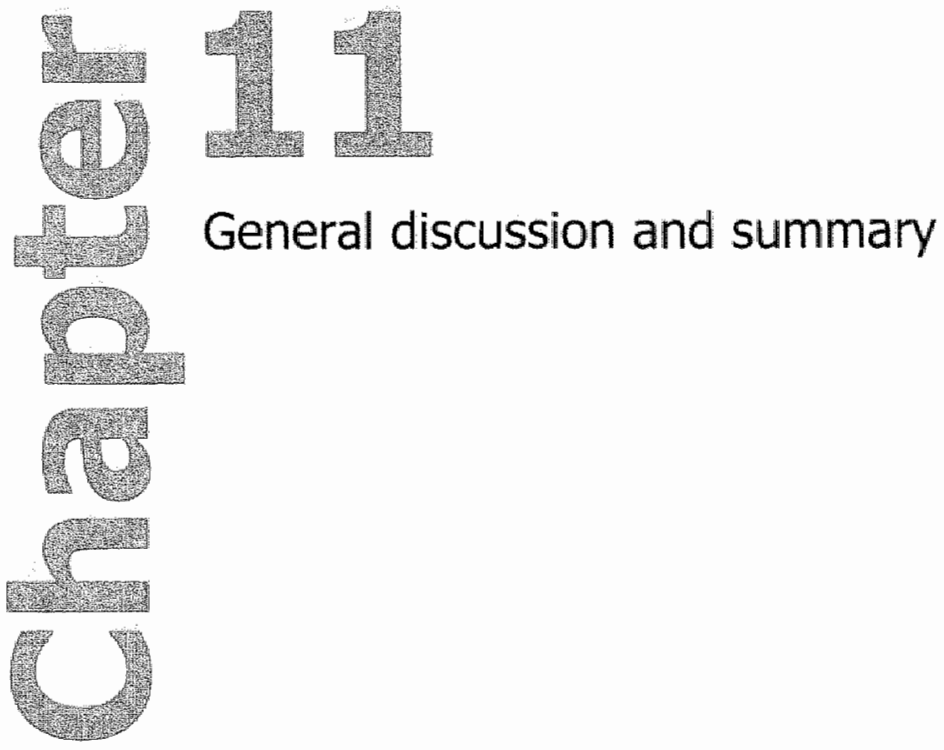


\section{General discussion}

Despite a multitude of publications on the morphology and function of the sole of the foot, the resulting contemporary image is incomplete and sometimes confusing. Besides, some fundamental concepts that are used in interpreting (diagnostic) tests and in planning therapeutic interventions, were never systematically tested. The possible value of this thesis lies not so much in the fundamental issues it addresses or in information it adds to the contemporary knowledge of the sole, but more in the change of the general concept on the function of the sole it offers. Putting the various elements of the previous chapters together, the sole of the foot emerges as a highly organized and differentiated structure that helps to protect the rest of the body against injury. It proved to be an integral part of the foot, where regional differences in functional requirements are expressed in morphological and mechanical differences.

During locomotion, the bones of the fore foot "freely" move around in the soft tissue of the sole in both vertical and horizontal directions. In the horizontal directions (including the deformation by torque), the extent of this movement is limited by various connective tissue structures that tether the skin and subcutaneous soft tissue of the sole to the deeper layers of the foot (chapter 2). MRI displayed this limitation in the antero-posterior direction. Thus, the internal connective tissue structures provide stability to the sole of the foot with the preservation of the necessary mobility to adapt to irregularities of the ground. Moreover, mobility helps to disperse the repetitive loadings of locomotion over a larger area. In this sense, mobility of the skin relative to the bones helps to protect the skin from injury. In addition to the already mentioned properties, the internal connective tissue structures seem to be able to (passively) deflect the loadings away from the most vulnerable parts of the bones of the foot (chapter 4).

The vertical down shift of the bones into the soft tissue of the sole is important in damping the impact forces of locomotion. In the unloaded situation, the thickness of the soft tissue under the metatarsal heads increases from lateral to medial. At progressive loadings of the forefoot, the sole of the foot progressively gets thinner, but the mutual position of the metatarsal heads persists. The loadings on the forefoot are centrally and medially higher than laterally. The soft tissue keeps the metatarsal heads in place; its thickness illustrates the principle of adequate cushioning, not the existence of a functional distall transverse arch (chapter 5). To facilitate the vertical down shift of the metatarsals, the pliable subcutaneous tissue is displaced to the borders of the foot, similar to the widening of the heel pad during loading as was reported by others. Moreover, some tissue is shifted dorsally in the intermetatarsal spaces, as the loading causes the metatarsal bones to sink into the sole. This mechanism may add to the damping power of the forefoot (chapter 3).

Also compression of the venous plexus of the forefoot added to the vertical down shift of the metatarsal heads as made plausible by the decrease in volume of the plantar side of the forefoot after loading. Because of the large magnitude of the decrease, we hypothesized that the venous plexus functions as a hydro-mechanical damper. Although we confirmed this hypothesis at the heel region with pendulum impact tests, the venous plexus only marginally contributed to the damping power of the sole (chapter 9). In the forefoot, also deep venous structures are present. However, a similar test on the forefoot did not demonstrate a contribution of the venous plexus to the damping power. Probably, this was due to the difficulties in stabilizing the forefoot during impact, which limited the accuracy (chapter 10). The fundamental visco-elastic properties of biomaterial predict a change in the mechanical behaviour on repeated impacts. Because we used repeated impacts to evaluate the effects of venous congestion, we also had to analyse the effect of repeated impacts as such. Earlier studies, using a similar type of in vivo experiments, reported no effect of repeated impacts. At lower impact velocities, we confirmed the visco-elastic properties in the increase of the elasticity of the padding on consecutive impacts (chapter 8). Moreover, we found a velocity 
dependency that could not exclusively be explained by the fundamental properties. This velocity dependency seemed to support the recent suggestion that the muscles of the lower leg can actively anticipate and adapt to the next impact in a series of impacts as in locomotion. This 'tuning' of the muscles is directed to increase the damping power of the lower leg system.

We also compared the mechanical properties of the forefoot and the heel (chapter 10). The impacts on the heel were significantly more elastic. Because the structure of the sole at the forefoot and the heel differ only in detail, the presence of the deeper lavers should explain the difference. Together with the observations that the presence of the lower leg increases the damping of an impact, we have come to the insight that attenuation of an impact not only involves the padding of the foot, but also involves a much larger area. This observation explains the sometimes contradictory reports on the mechanics of the heel, but also illustrates the complexity of the field of the mechanics of locomotion.

Although we painted a dynamic picture of a continuously adapting sole of the foot on loading, we realise that some caution in this interpretation is in place. In fact, the foregoing description originates from sequential static positions, not from truly dynamic events of normal locomotion. Moreover, the presented results originate from normal subjects. Therefore, one should be cautious to extrapolate our observations to pathological conditions such as diabetes mellitus or to the field of designing shoes or inlays. Nevertheless, the results can be used as a starting point of future investigations.

\section{Summary}

To meet the requirements of locomotion, the sole of the foot is pliable, resillient, and tough. In chapter 2 , the underlying internal structures were described as visualized by MRI and they were compared with contemporary anatomic insights. In general, the anatomy that MRI displayed largely was in line with the descriptions of traditional anatomy. However, MRI not only showed some additional structures, but also gave more insight in the relation between the internal structures, and stability and integrity of the sole of the foot.

The third to the seventh chapter deal with one experiment that was carried out on ten healthy volunteers, from different perspectives. The experiment combined morphology as depicted by computed tomography with plantar pressure distributions as registered by a pressure measuring inlay.

In chapter 3 we described the changes in the distribution of soft tissue in the forefoot on loading. The most prominent result was a decrease of the volume on the plantar slide $(4.8 \%)$ that was most likely due to the compression of plantar venous structures. Besides, a small increase in volume (1.6\%) on the dorsal side likely reflected a dorsal shift of soft tissue in the intermetatarsal spaces. Both observations are potentially important in relation to shock absorption and they initiated the complementary study on which chapter eight to ten reported.

In clinical and experimental studies, the unspoken premise that peak plantar pressure is located directly under the bony prominences in the forefoot is used. Even therapeutic interventions are based on this premise. In chapter 4 the lhypothesis was put to the test. It: showed that the position of the metatarsal heads 1,4 , and 5 and the position of local peak plantar pressure differed significantly. The findings suggest that normal plantar soft tissue is able to deflect a load away from the most vulnerable structures. The observations improve 
insight in the function of the normal forefoot and might direct further research on pathogenesis and therapeutic intervention of complaints of the forefoot.

Although the existence of a transverse arch at the level of the heads of the metatarsals is generally denied, it is still a matter of debate. In chapter 5 , we investigated the matter by describing the relation between the position of the metatarsal heads as indicated by the thickness of the sole underneath, and the corresponding plantar pressures during different degrees of loading. Excluding the sesamoids, the thickness of the sole under the bones of the forefoot increased from lateral to medial. This relation persisted at all degrees of loading. Including the sesamoids in the load bearing bony structures, the bones were placed in geometrical arch; just the same they did not form a functional arch. The soft tissue underneath the heads kept them in place; its thickness reflected the principle of adequate cushioning.

In preparation to study the effect of loading of the forefoot on the position of the metatarsals with the data of our CT experiment, it was noticed that most reports on metatarsal splaying used angular measurements on radiographs. However, the distortion that comes with radiographic projection of bones was never systematically investigated. In chapters 6 and 7 radiography was simulated to quantify radiographic distortion in angular measurements in the forefoot. We used a mathematical model for a theoretical analysis and our CT-data to study distortion in dailly practice.

The distortion of the declination angles of the metatarsals on the lateral radiograph was practically negligible. The variability of distortion of the intermetatarsal angles on the anteroposterior radiograph was only about one third of the interobserver variability. However, they may have cumulative effects on the error of measured intermetatarsal angles and should be considered (chapter 6 ).

The distortion due to angulation of the roentgen tube was so small, that for practical reasons, a $15^{\circ}$ cranio-caudal tube angulation was recommended as the standard technique to obtain an antero-posterior radiograph of the forefoot (chapter 7).

From the eighth to the tenth chapter, the results of impact experiments were presented. In the experiments a pendulum collided with the sole of the foot of healthy volunteers. During the impact, the deceleration of the pendulum was recorded with an accelerometer that was firmly attached to the back of the bar of the pendulum. From these recordings, loaddeformation curves were derived. Four parameters characterized the individual curves. Changes in the magnitude of the parameters before and after an intervention described the effect.

Primarily, we were interested in the contribution of the plantar venous structures to the damping power of the sole of the foot. Because this only could be determined by sequential measurements, we also had to take the (possible) effect of repeated impacts into account.

Chapter 8 reports on this effect of repeated impacts on the heel region. Overall, repeated collisions resulted in more elastic impacts, characterized as a rise of maximum force and maximum indentation and a decline of dissipated energy and stiffness (all in the order of $3 \%$ ). If a parameter showed changes in magnitude, most of the change was already reached after the very first impacts. The fundamental visco-elastic properties of the heel pad explain most of these observations. However, the significant decrease of the maximum force and stiffness on consecutive impacts at higher impact velocities do not match to the visco-elastic properties. We suggested that these observations expressed the adaptation of the muscles of the calf to the impact force, also referred to as "tuning". We concluded that the damping effect of the lower leg more than compensated the loss of damping power of the heel pad on repeated impacts. 
In chapter 9 we tested the hypothesis that the venous plexus might contribute as a hydraulic shock absorber to the damping properties of the heel pad. At congestion of the plantar veins, the maximum impact force decreased slightly but significantly by $2.6 \%$ at $0.2 \mathrm{~m} / \mathrm{s}$ and $1.8 \%$ at $0.4 \mathrm{~m} / \mathrm{s}$. This effect was no longer found at $0.6 \mathrm{~m} / \mathrm{s}$. Although these effects are rather small, they confirm the fundamental hypothesis that the venous plexus contributes to the damping properties of the heel pad during walking.

To our knowledge, presently no data are available on the damping properties of the forefoot. In chapter 10, we reported on pendulum impact tests on the forefoot similar to previous experiments on the heel with one and the same group of volunteers. The goal of this study was to explore whether the forefoot also showed effects of venous congestion and repeated impacts as observed in the heel region. Moreover, we wanted to compalre the magnitude of the parameters that described the impacts on the heel region with those on the forefoot. The results did not show a significant contribution of wenous congestion to the damping abilities of the sole. The effect of repetitive impacts was confirmed and results paralleled those of the heel. The mechanical characteristics were significantly different at the forefoot compared to those of the heel region. Most likely, the differences in anatomy of the forefoot and the heel explain this result rather than an intrinsic difference in the mechanical properties of the soft tissue of the sole of the foot. The results illustrate that the damping of an impact is not a local phenomenon, but involves the underlying structures as well. 


\section{Algemene beschouwing en samenvatting}

\section{Algemene beschouwing}

Ondanks vele publicaties, is het huidige beeld van de vorm en functie van de voetzool incompleet en soms zelfs verwarrend. Bovendien zijn fundamentele concepten die gebruik: worden bij het interpreteren van (diagnostische) onderzoekingen en het plannen van behandellingen nooit systematisch onderzocht. In dit proefschrift zijn een aantal onderzoeken beschreven die betrekking hebben op deze fundamentele zaken. De betekenis van dit proefschrift moet echter eerder gezocht worden in de gehanteerde benadering van de functie van de voetzool dan in de verdiepende fundamentele kennis. De in dit proefschrift beschreven studies creèren cen beeld wan de voetzool als een sterk georganiseerde en gedifferentieerde structuur, die helpt de rest van het lichaam te beschermen. De zool blijkt een integraal deel van de voet te zijn, waarvan regionale verschillen in functie tot uiting komen in verschillen in vorm en mechanische eigenschappen.

Tijdens het lopen, bewegen de botten van de voorvoet voortdurend ten opzichte van de weke delen van de zool, zowel in horizontalle als in verticale richting. In horizontale richting (inclusief de vervorming door draaiing) wardt de verschuiving beperkt door verschillende bindweefselstructuren die de huid en de onderhuidse weke delen verbinden met de diepere lagen van de voet (hoofdstuk 2). MRI illustreerde dit voor de voorachterwaartse verschuiving. Daarnaast geven de onderhuidse bindweefselstructuren stevigheid aan de zool met behoud van de nodige beweeglijkheid on zich aan te passern aan onregeimatigheden op de grond. Bovendien helpt de beweeglijkheid om de zich herhalende belasting tijdens het lopen te verspreiden over een groter oppervlak van de zool. Hierdoor wordt de huid beschermd tegen beschadiging. Naast de reeds genoemde eigenschappen, lijken de inwendige bindweefselstructuren de krachten die tijdens lopen optreden af te kunnen buigen van de meest kwetsbare delen van het voetskelet (hoofdstuk 4).

De neerwaartse verplaatsing van de botten in de weke delen van de zool is belangrijk bij het dempen van krachten die optreden tijdens het landen bij lopen en rennen. In een onbelaste toestand, neemt de dikte van de zool onder de kopjes van de middenvoetsbeentjes van buiten naar binnen toe. Centraal en aan de binnenkant van de woorvoet is de belasting groter dan aan de buitenkant. Bij toenemende belasting, wordt de zool dunner, maar de onderlinge positie van de kopjes van de middenvoetsbeentjes verandert niet. De weke delen van de zool blijken de kopjes op hun plats te houden. De dikte wan de weke delen van de zool onderstreept de noodzaak tot demping op plaatsen van de grootste belasting, maar is geen argument voor het bestaan van een functioneel voorste dwarse voetgewelf (hoofdstuk 5). Om de neerwartse verplaatsing van het voetskelet mogelijk te maken, wordt het soepele onderhuidse weefsel naar de voetranden verplaatst. Bovendien verschuift er weefsel tussen de middenvoetsbeentjes naar de rug van de voet, terwijl de middenvoetsbeentjes zelf in de voetzool zinken. Dit mechanisme kan een aanvullende roll spelen in het dempende vermogen van de voorvoet (hoofdstuk 3).

Ook het leegdrukken van aderen in de voarvoet helpt bij de neerwaartse verplaatsing van de kopjes van de middenvoetsbeentjes, zoals waarschijnlijk gemaakt werd door het volumeverlies van de weke delen aan de plantaire zijde van de voet na het belasten. Vanwege de grootte van dat volumeverlies, hypothetiseerden we dat de ader werkte als een hydromechanische demper. Hoewel we de hypothese konden bevestigen met botsingsproeven van een stinger in de hielregio, was het dempende effect slechts marginaal. Een soortgelijke test op de voorvoet toonde in het geheel geen effect op de demping van de botsing. Dit werd waarschijnlijk verklaard door een mindere nauwkeurigheid van de metingen omdat de voorvoet tijdens de botsingsexperimenten moeilijker te stabiliseren was (hoofdstuk 10). 
De fundamentele visco-elastische eigenschappen wan natuuilijke materialen voorspellen een verandering in mechanisch gedrag na herhaalde botsingen. Omdat we een reeks wan opvolgende botsingen gebruikten om het effect van stuwing van de aderen te onderzceken, moesten we ook het effect van herhaalde botsingen als zodanig onderzoeken. Eerdere studies van andere auteurs, die van eenzelfde type onderzoek gebruik maakten, rapporteerden dat er geen effect was van herhaalde botsingen op demping. Bij lagere botsingssnelheden konden wij de visco-elastische eigenschappen bevestigen, omdat er een toename van de elasticiteit van het weefsel van de zool werd vastgesteld (hoofdstuk 8). Echter, de fundamentele weefseleigenschappen konden niet verklaren dat herhaalde botsingen een toename lieten zien van het dempende vermogen van de zool bij toenemende botsingssnelheid. Deze relatie lijkt de recente suggestie te ondersteunen, dat de spieren van het (onder)been zich actief kumnen voorbereiden op de komende 'klap' in een serie van botsingen zoals tijdens lopen. Deze 'fijnafstelling' van spieren zou het dempende vermogen van het onderbeen-systeem vergroten.

Tevens vergeleken we de mechanische eigenschappen van de voorvoet en de hiel (hoofdstuk 10). De botsingen op de hiel waren significant elastischer. Omdat de structuur van de voetzool van de hiel en de voorvoet slechts in detail van elkaar verschillen, moet de aanwezigheid van diepere structuren de gevonden verschillen verklaren. Samen met de waarneming dat de aanwezigheid van het onderbeen de demping van een botsing versterkt, zijn wij tot het inzicht gekomen dat een botsing niet alleen de locale weke delen treft, maar een veel groter gebied. Deze vaststelling verklaart de soms tegenstrijdige berichten over de mechanica van de hiel, maar het illustreert tegelijkertijd de complexiteit van de mechanica van het lopen.

Hoewel we een dynamisch beeld hebben geschetst van een zich continu aanpassende voetzool tijdens belasting, realiseren we ons dat enige reserve bij deze interpretatie op zijn plaats is. Elgenlijk vindt de bovenstaande beschrijving niet zijn oorsprong in echte dynamische bewegingen, maar in opeenvolgende statische posities. Bovendien zijn de verkregen resultaten afkomstig van onderzoek aan gezonde proefpersonen. Daarom dient men zorgvuldig en terughoudend te zijn bij het extrapoleren van onze resultaten naar pathologie zoals diabetes mellitus, of naar het veld van het ontwerpen van schoenen of steunzolen. Desalniettemin kunnen de resultaten een uitgangspunt vormen voor toekomstig onderzoek.

\section{Samenvatting}

Om te kunnen lopen of rennen, moet de voetzool soepel, veerkrachtig en tegelijkertijd taai zijn. In hoofostuk 2 worden de inwendige bindweefselstructuren beschreven zoals MRI die zichtbaar maakt. Deze beelden worden vergeleken met de huidige anatomische inzichten. In het algemeen komt de anatomie, zoals die zichtbaar wordt met MRI overeen met de beschrijvingen van de traditionele anatomie. Echter, MRI toonde niet alleen enkele nieuwe structuren, maar gaf ook meer inzicht in de onderlinge samenhang van diverse inwendige structuren en in de stabiliteit en integriteit van de voet.

In hoofdstuk 3 tot en met 7 wordt een experiment op tien gezonde proefpersonen vanuit verschillende invalshoeken behandeld. Het experiment combineerde computertomografie en drukmeting met behulp van een drukgevoelige inlegzool. Zo kon de relatie tussen de vorm van de voet en de drukverdeling onder de voetzool worden vastgesteld.

In hoofdstuk 3 beschrijven wij de veranderingen in de verdeling van de weke delen van de voorvoet door belasting. Het meest opvallende resultaat was een afname van het volume 
van de voetzool $(4,8 \%)$, die hoogst waarschijnijk grotendeels het gevolg was van het leegdrukken van aderen. Bovendien, werd er een kleine volumetoename $(1,6 \%)$ van de woetrug wastgesteld; die verklaard kan worden door een verplaatsing van weefsel in de ruimte tussen de middenvoetsbeentjes. Beide wamemingen kunnen in principe van belang zijn bij schokabsorptie en zij vormden de aanleiding voor aanvullend onderzoek dat in het achtste tot het tiende hoofdstuk wordt beschreven.

In klinische en experimentele studies wordt impliciet de veronderstelling gebruikt dat precies onder het botstuk dat het diepst in de woetzool staat, de hoogste plantaire druk is gelegen. Zelfs therapieen maken van deze veronderstelling gebruik. In hoofdstuk 4 werd de hypothese getest. De positie van de kopjes van de middenvoetsbeentjes 1, 4, en 5 en de positile van de hoogste locale plantaire druk bleken significant van elkaar te verschillen. Deze bevinding suggereert dat het weefsel van de voetzool in staat is belasting van de meest kwetsbare structuren af te leiden. Deze waarneming vergroot het inzicht in de functie van de normale voorvoet en kan een uitgangspunt zijn voor toekomstig onderzoek naar het ontstaan wan zlekte en het ontwikkelen van behandelingen bij klachten van de voorvoet.

In voorbereiding op een analyse van het spreiden van de voorvoet bij belasting met onze gegevens van het CT-experiment, viel ons het veelvuldige gebruik van hoekmetingen op röntgenfoto's in de bestaande literatuur op. Echter, de vertekening die ontstaat door radiologische projectie van botten is nooit systematisch onderzocht. In de hoofdstukken 6 en 7 werd de invloed van de radiologische vertekening op hoekmeting van de middenvoetsbeentjes gekwantificeerd door de radiologische opnametechniek te simuleren. Hiervoor gebruikten we een wiskundig model voor theoretische analyse en onze gegevens van thet CT-experiment voor representatieve analyse van de dagelijkse praktijk. Voor de zijdelingse röntgenfoto bleek de vertekening van de declinatiehoeken van de middenvaetsbeentjes praktisch verwaarloosbaar. Voor de voorachterwaartse röntgenfoto, bedroeg de varlantie van de vertekening van de hoeken tussen de middenvoetsbeentjes slechts een derde van de variantie van de waarnemers onderling, zoals die door andere auteurs was vastgesteid. Desondanks, dient rekening gehouden te worden met vertekening, omdat vertekening en de waarnemerfout een cumulatief effect kunnen hebben op de fout in de hoekmeting (hoofdstuk 6).

De vertekening als gevolg van angulatie van de röntgenbuis bleek zo klein te zijn, dat we, om praktische redenen, toch een standaard techniek voor de voorachterwaartse röntgenfoto aanbevelen met een cranio-caudlale buisangulatie van $15^{\circ}$ (hoofdstuk 7).

De resultaten van de botsingsproeven worden im hoofdstuk 8 tot en met 10 gepresenteerd. Bij deze experimenten botst het gewicht van een slinger tegen een deel van de voetzool wan gezonde vrijwilligers. Een accelerometer die stevig op de achterzijde van het gewicht was gemonteerd, registreerde de versnelling van het gewicht tijdens de botsing. Van deze registratie werden krommes afgeleid die de belasting als functie van de indeuking weergaven. We kozen vier variabelen om een kromme te karakteriseren. De veranderingen in de grootte van een dergelijke variabele na een interventie werden als maat voor het effect genomen. In eerste instantie waren we geïnteresseerd in de bijdrage van aderen aan het dempende vermogen van de voetzool. Omdat dit alleen kon worden vastgesteld door gedurende langere tijd herhaaldelijk botsingen te meten, moesten we ook rekening houden met de mogelijkheid dat herhaling van botsing op zich ook al een effect kom hebben. Hoofdstuk 8 rapporteert ower het effect van herhaalde botsingen tegen de hiel. In het algemeen leidt herhaling tot meer elastische botsingen, die gekarakteriseerd worden door een toename van de maximale kracht en maximale indeuking en een afname van de gedissipeerde energie en stijfheid (alle effecten ongeveer $3 \%$ ). Als een variabele een verandering toonde, was het grootste deel van het effect al na de eerste botsingen bereikt. 
De fundamentele visco-elastische eigenschappen van de voetzool van de hiel kunnen een deel deze waarnemingen verklaren. Een significante afname van de maxmale kracht en stijfheid bij opwolgende botsingen bij hogere botsingssnelheden klopten echter niet met de veronderstelde visco-elastische eigenschappen. Wij suggereerden dat deze waarnemingen een uiting zijn van de aanpassing wan de spieren in de kuit aan de kracht wan een botsing, in de literatuur ook wel 'fijnafstemming' genoemd. Wij concluderen dat het dempende effect van het onderbeen meer dan compenseerde voor het verlies van thet dempende vermogen van de zool van de hiel bij herhaalde botsingen.

In hoofistuk 9 hebben we de hypothese getest dat de aderen van de woetzool als een hydromechamische demper kunnen bijdragen aan het dempende eigenschappen van de zool van de hiel. Door de aderen te stuwen, nam de maximale kracht die tijdens de opvolgende botsing werd gemeten slecht $2,6 \%$ bij $0,2 \mathrm{~m} / \mathrm{s}$ en $1,8 \%$ bij $0,4 \mathrm{~m} / \mathrm{s}$ af, Deze resultaten waren significant. Bij $0,6 \mathrm{~m} / \mathrm{s}$ werd geen effect meer vastgesteld. De fundamentele hypothese dat de aderen van de voetzool bijdragen aan dempende eigenschappen van de zool van de hiel tijdens wandelen worden hierbij bevestigd, ondanks de kleine omvang van de effecten.

Voor zover wij weten, zijn er momenteel geen gegevens beschikbaar over de dempende eigenschappen van de voorvoet. In hoofdstuk 10 rapporteren wij over botsingstesten tegen de zool van de voorvoet, die lijken op de experimenten die bij de hiel werden uitgevoerd op dezelfde groep vrijwilligers. Het doel van de studie was te onderzoeken of de voorvoet ook effecten van stuwing van aderen en van herhaalde botsingen vertoont, zoals reeds was vastgesteld in de hiel. Bovendien wilden we de grootte van de variabelen die een botsing karakteriseren in de hiel vergelijken met die in de voorvoet. De resultaten toonden geen significante bijdrage wan de stuwing van de aderen aan de dempende eigenschappen van de voorvoet. De effecten van herhaalde botsingen werden bevestigd en kwamen met de resultaten van de hiel overeen. De mechanische karakteristieken waren in de voorvoet en in de hiel significant verschillend. Waarschijnlijk worden de verschillen beter verklaard door anatomische verschillen tussen de voorvoet en de hiel dan door intrinsieke verschillen in de weke delen wan de voet. De resultaten illustreren dat de demping van een impact niet een lokaal fenomeen is, maar dat ook de onderliggende structuren van belang zijn. 


\section{Curriculum vittae}

Rene Erik Weijers

born

place of birth

marited to

$1972-1978$

$1978-1985$

$1985-1989$

$1989-1991$

$1991-$ november $26^{4}, 1959$

's-Gravenhage, the Metherlands

Jacqueline Weijers-Horree, two children, Bas and Tom

Atheneum- $\beta$, Cum Laude, scholengemeenschap Simon Stevin, 's-Gravenhage

Medicine at the University of Leiden, Cum Laude

Resident at the department of Radiology, University Hospital Groningen

Chairman: Prof. Dr. C.J.P. Thijn

Staff member at the department of Radiology, University Hospital Groningen

Staff member at the University Hospital Maastricht

Chairman: Prof. Dr. J.M.A. van Engelshoven 


\section{Dankwoord}

$\mathrm{Na}$ vijf jaren, $2 \mathrm{~Gb}$ aan getallen, 2 computers, drie harde schijven, zes kilo gips, en een stapel afvalhout is het dan zover. Ik kan er een punt achter zetten, maair niet voordat lk een ieder die aan dit proefschrift heeft bijgedragen bedank. Als eerste gaat mijn dank uit naar mijn co-promotor, promotoren en medeauteurs, als naaste betrokkenen. Ook de proefpersonen die geheel belangeloos aan de experimenten hebben meegewerkt, wil ik hierbij bedanken.

Ik heb ondervonden dat wetenschap bedrijven meer is dan onderzoeken en publiceren. Hek is overleggen, plannen, schipperen en laveren tussen de voortgaande patiëntenzorg en de wetenschap. In deze spagaat zijn binnenbochten en ontwijkende acties soms onvermijdelijk: Het vereist begrip van de ander waarmee je wilt samerwerken. Daarom gaat mijn dank ook uit naar al diegenen die soms -zonder de achtergrond van mijn handelen te kennen-, minder kregen dan ze mochten verwachten. Zonder jullie was dit proefschrift nooit tot een afronding gekomen.

Als laatste will ik mijn familie en vrienden bedanken, maar bovenal Lientje, Bas en Tom voor hun onvoorwaardelijke steun. Vaak was ik wel aanwezig, maar was ik er niet. Ik hoop oprecht dat daar verandering in komt en dat jullie mij de kans geven om 'verloren' tijd in te halen. 